\title{
Search for Low-Mass Dijet Resonances Using Trigger-Level Jets with the ATLAS Detector in pp Collisions at $\sqrt{s}=13 \mathrm{TeV}$
}

\author{
M. Aaboud et al." \\ (ATLAS Collaboration)
}

(Received 11 April 2018; published 22 August 2018)

\begin{abstract}
Searches for dijet resonances with sub-TeV masses using the ATLAS detector at the Large Hadron Collider can be statistically limited by the bandwidth available to inclusive single-jet triggers, whose data-collection rates at low transverse momentum are much lower than the rate from standard model multijet production. This Letter describes a new search for dijet resonances where this limitation is overcome by recording only the event information calculated by the jet trigger algorithms, thereby allowing much higher event rates with reduced storage needs. The search targets low-mass dijet resonances in the range $450-1800 \mathrm{GeV}$. The analyzed data set has an integrated luminosity of up to $29.3 \mathrm{fb}^{-1}$ and was recorded at a center-of-mass energy of $13 \mathrm{TeV}$. No excesses are found; limits are set on Gaussian-shaped contributions to the dijet mass distribution from new particles and on a model of dark-matter particles with axial-vector couplings to quarks.
\end{abstract}

DOI: 10.1103/PhysRevLett.121.081801

Introduction.-If new particles beyond those of the standard model (SM) are directly produced in protonproton $(p p)$ collisions at the Large Hadron Collider (LHC), they must interact with the constituent partons of the proton, and can therefore also decay into the same partons, resulting in two-jet final states. Quantum chromodynamics (QCD) predicts that dijet events have an invariant mass distribution $\left(m_{j j}\right)$ that falls smoothly, whereas a new state decaying to two partons would emerge as a localized excess in the distribution.

Traditional dijet searches at the LHC focus on the production of heavy particles with masses above $900 \mathrm{GeV}$ [1-3]. LHC searches for lighter resonances with small production cross sections have been hampered by restrictions in the data-taking rate of the ATLAS and CMS detectors. Single-jet triggers with a jet $p_{\mathrm{T}}$ threshold below roughly $380 \mathrm{GeV}$ are prescaled, a procedure whereby only a fraction of the events passing the trigger are recorded; hence, dijet events with an invariant mass below $1 \mathrm{TeV}$ are largely discarded by the trigger system, as indicated in Fig. 1. Therefore, despite the large number of $p p$ collisions produced by the LHC, traditional ATLAS and CMS searches are less sensitive to dijet resonances below $900 \mathrm{GeV}$ than searches at the SPS and Tevatron colliders [4-9]. Alternative trigger strategies to search for low-mass resonances include selecting events with jets recoiling

*Full author list given at the end of the article.

Published by the American Physical Society under the terms of the Creative Commons Attribution 4.0 International license. Further distribution of this work must maintain attribution to the author(s) and the published article's title, journal citation, and DOI. Funded by SCOAP ${ }^{3}$. against either an energetic photon or an additional energetic jet [10-12], or selecting events with decays to heavy-flavor jets $[13,14]$. In these cases, additional features in the events reduce the data-taking rates, reducing the sensitivity to lowmass resonances.

This Letter describes an innovative data-taking approach to access the invariant mass region below $1 \mathrm{TeV}$; only a reduced set of information from the trigger system is recorded and subsequently analyzed. The Trigger-object Level Analysis (TLA) approach allows jet events to be recorded at a peak rate of up to twice the total rate of events using the standard approach, while using less than $1 \%$ of

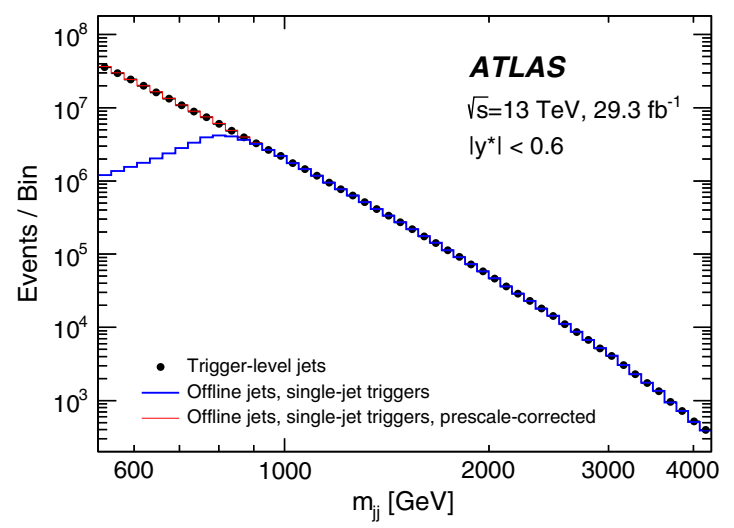

FIG. 1. Comparison between the number of dijet events in the data used by this analysis (black points), the number of events selected by any single-jet trigger (thicker, blue line), and the events selected by single-jet triggers but corrected for the trigger prescale factors (thinner, red line) as a function of the dijet invariant mass $\left(m_{j j}\right)$. The definition of $y^{*}$ is $\left(y_{1}-y_{2}\right) / 2$, where $y_{1}$ and $y_{2}$ are the rapidities of the highest- and second-highest $p_{\mathrm{T}}$ jets. 
the total trigger bandwidth [15]. This strategy is used in dijet resonance searches in $\sqrt{s}=8$ and $13 \mathrm{TeV}$ LHC $p p$ collision data by the CMS Collaboration [16,17], and it is used by the LHCb Collaboration (e.g. [18]). The analysis presented here uses $29.3 \mathrm{fb}^{-1}$ of $\sqrt{s}=13 \mathrm{TeV} p p$ collision data recorded in 2016 by the ATLAS detector.

ATLAS detector and data sample.-The ATLAS detector [19] is a multipurpose detector with a forward-backward symmetric cylindrical geometry and nearly $4 \pi$ coverage in solid angle [20], consisting of tracking detectors, calorimeters, and a muon spectrometer. In the pseudorapidity region $|\eta|<3.2$, high-granularity lead and liquid-argon (LAr) electromagnetic sampling calorimeters are used. A steel and scintillator hadronic tile calorimeter provides coverage in the range $|\eta|<1$.7. Hadronic calorimetry in the endcap region, $1.5<|\eta|<3.2$, and electromagnetic and hadronic calorimetry in the forward region, $3.1<|\eta|<4.9$, are provided by LAr sampling calorimeters. A two-level trigger system is used to select events for offline storage [15]. A first-level (L1) trigger based on dedicated hardware identifies jets from $\Delta \eta \times \Delta \phi=0.2 \times 0.2$ calorimeter segments with a sliding-window algorithm. Events passing the L1 trigger are processed by a software-based high-level trigger (HLT). The HLT system reconstructs jets using the anti- $k_{t}$ algorithm $[21,22]$ with radius parameter $R=0.4$. The inputs to this algorithm are groups of contiguous calorimeter cells (topological clusters), in which each cell's inclusion is based on the significance of its energy deposit over calorimeter noise [23]. Jet four-momenta are computed by summing over the four-momenta of the topological clusters that compose the jet, with each cluster pointing to the center of the ATLAS detector and being treated as massless. The HLT jet reconstruction uses the same techniques that the ATLAS offline jet reconstruction applies to similar inputs from recorded data events that include the full detector information [15].

After execution of the HLT jet algorithm, only triggerlevel jets with $p_{\mathrm{T}}>20 \mathrm{GeV}$ are stored. The stored information includes the four-momentum of each jet and a set of calorimeter variables characterizing the jet [24], such as information about the jet quality and structure. The average size of these events is less than $0.5 \%$ of the size of full offline events that contain all detector information. For this analysis, all events containing at least one L1 jet with $E_{\mathrm{T}}>100 \mathrm{GeV}$ are selected and recorded, corresponding to a total luminosity of $29.3 \mathrm{fb}^{-1}$. Events containing a L1 jet with $E_{\mathrm{T}}>75 \mathrm{GeV}$ are also selected, in a subset of these data corresponding to $3.6 \mathrm{fb}^{-1}$ that were collected at the start of the data-taking period. Events with at least one L1 jet with $E_{\mathrm{T}}>100 \mathrm{GeV}$ are therefore included in both data sets.

Calibration procedure.-After the events are recorded, the trigger-level jet energy and direction are corrected to those of simulated particle-level jets built from stable particles with a lifetime longer than $10 \mathrm{ps}$, excluding

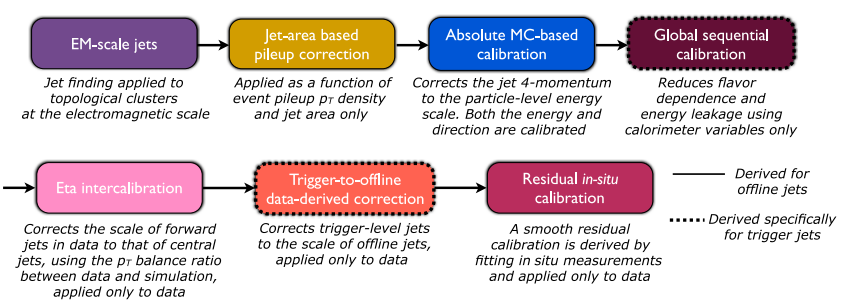

FIG. 2. Calibration stages for EM-scale trigger-level jets, each applied to the four-momentum of the jet. MC refers to the simulation.

muons and neutrinos. Before any calibration, the jet $p_{\mathrm{T}}$ response, defined as the $p_{\mathrm{T}}$ ratio of a trigger-level jet to the same jet [25] reconstructed offline (offline jet), is between 0.95 and 1.05 in the $p_{\mathrm{T}}$ range considered by this analysis. Since the energy scale for trigger-level and offline jet are very similar, the trigger-level jet calibration employs the same procedure and constants as derived for offline jets [26], with some modifications to account for the unavailability of tracking information for trigger-level jets.

In the calibration procedure, summarized in Fig. 2, an event-by-event jet-area-based calibration [27] is used to correct for contributions from additional proton-proton interactions (pileup) in the same and neighboring crossings of proton bunches. Then, the simulation-based calibration derived for offline jets is applied to trigger-level jets to correct both jet energy and direction. Next, calorimeterbased variables are used to reduce the dependence on the trigger-level jet flavor and to minimize the impact of energy leakage. Only variables related to the trigger-level jet energy fractions in the electromagnetic and hadronic calorimeters and the minimum number of calorimeter cells containing $90 \%$ of the trigger-level jet energy are used here since track-based variables, which are normally used in the offline calibration, are not available. With this correction, the trigger-level jet energy resolution is improved by $8 \%$ at jet $p_{\mathrm{T}}$ values of $85 \mathrm{GeV}$ and up to $40 \%$ for jet $p_{\mathrm{T}}$ values of $1 \mathrm{TeV}$ relative to the previous calibration step. Next, the calibration corrections that restore the relative calibration between central and forward jets in data and simulation are derived for offline jets and applied to trigger-level jets. After these calibration steps, any residual difference between trigger-level jets and offline jets is accounted for in a dedicated correction, based on the $p_{\mathrm{T}}$ response and derived from data in bins of jet $\eta$ and $p_{\mathrm{T}}$. The size of this correction is on average $1 \%$, with values reaching up to $4 \%$ in the endcap regions of the calorimeter.

Finally, an in situ calibration is obtained from the datato-simulation ratio of the $p_{\mathrm{T}}$ balance between offline jets and well-calibrated objects against which the jets recoil. Three different types of well-calibrated objects are used to span the full $p_{\mathrm{T}}$ range of the jets: $Z$ bosons decaying to electrons or muons, photons, and multijets. A polynomial in $\log \left(p_{\mathrm{T}}\right)$ is simultaneously fit to the three input measurements to combine them. The resulting curve is taken as 


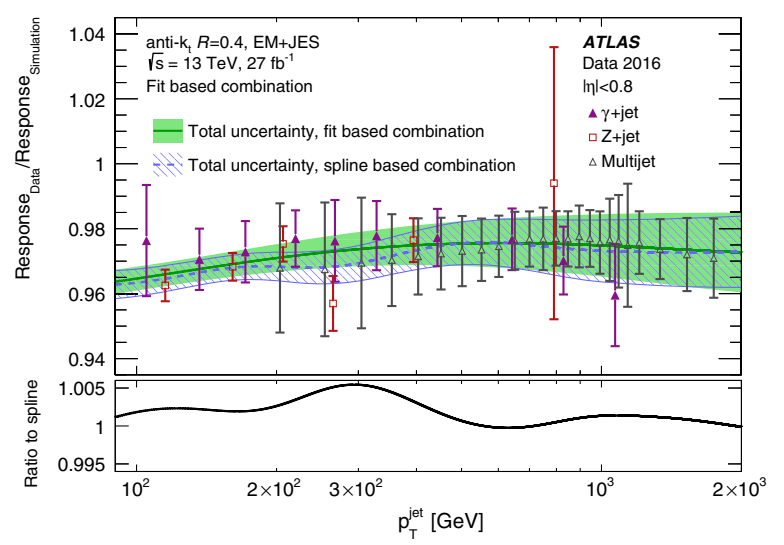

FIG. 3. The in situ calibration in the range $85 \mathrm{GeV}<$ jet $p_{\mathrm{T}}<2 \mathrm{TeV}$, for both the spline (dashed line) and fitted (solid line) combination methods, as described in the text. Data points from the three input measurements are overlaid. The lower panel shows the ratio of the two calibration curves.

the calibration correction to be applied to trigger-level jets. In deriving the final calibration curve, the fit is chosen over the simple spline-based combination procedure used for offline jets in Ref. [26]; this procedure is more robust against localized fluctuations in the jet $p_{\mathrm{T}}$ distribution that result in deviations from the expected smoothly falling invariant mass spectrum. Any dependence of the final mass spectrum on the parametrization of the jet energy scale calibration is tested by comparing different parametrizations on the data as well as on simulations. The fitted in situ calibration curve is compared to the spline-based smoothing procedure in Fig. 3. After the full calibration procedure, the energy of trigger-level jets is equivalent to that of offline jets to better than $0.05 \%$ for invariant masses of $400 \mathrm{GeV}$ and their difference is negligible for invariant masses of $1 \mathrm{TeV}$.

Energy scale and resolution uncertainties derived for offline jets [26] are applied to trigger-level jets in the signal simulation, with additional uncertainties equivalent to the size of the final trigger-to-offline correction (1\%-3\%). The uncertainty due to the modeling of pileup effects and due to the jet parton flavor are derived specifically for trigger-level jets and are comparable to those of offline jets. The jet energy scale uncertainty for trigger-level jets at $200 \mathrm{GeV}$ ranges from $1 \%$ at $|\eta|<0.8$ to $2 \%$ in the region between the central and endcap regions $(1.0<|\eta|<1.5)$.

Event selection.-The dijet event selection for this analysis is similar to the one used in Ref. [3]. Events must contain at least two trigger-level jets with $|\eta|<2.8$. The leading trigger-level jet must have either $p_{\mathrm{T}}>185 \mathrm{GeV}$ or $p_{\mathrm{T}}>220 \mathrm{GeV}$ for the $E_{\mathrm{T}}>75 \mathrm{GeV}$ and $E_{\mathrm{T}}>100 \mathrm{GeV}$ L1 trigger selections, respectively; this ensures that the L1 triggers are fully efficient. The second leading jet must have a $p_{\mathrm{T}}>85 \mathrm{GeV}$. Events that contain jets induced by calorimeter noise bursts, beam-induced background, or cosmic rays are rejected using the same criteria as in
Ref. [24], but omitting the track-based charged fraction selection, which is not available for trigger-level jets. This has a negligible effect for this analysis, since most of these backgrounds are already removed by requiring two central jets, as described below. The efficiency and purity of jets passing the selection are measured with a tag-and-probe method using data events with the full detector information. The trigger-level jet reconstruction efficiency is $100 \%$ for jets with $p_{\mathrm{T}}>85 \mathrm{GeV}$. The fraction of trigger-level jets that are not reconstructed and selected offline is below $0.1 \%$.

This analysis searches for a dijet resonance with a mass between 450 and $1800 \mathrm{GeV}$. Two different selection criteria are used for different but overlapping ranges of the $m_{j j}$ spectrum. To search for resonances with $700 \mathrm{GeV}<$ $m_{j j}<1800 \mathrm{GeV}$, events are required to have $\left|y^{*}\right|<0.6$, where $y^{*}=\left(y_{1}-y_{2}\right) / 2$ and $y_{1}$ and $y_{2}$ are the rapidities of the highest- and second-highest $p_{\mathrm{T}}$ trigger-level jets. To search for lower-mass resonances, with $m_{j j}>450 \mathrm{GeV}$, events with $\left|y^{*}\right|<0.3$ are selected from the smaller data sample requiring a L1 jet with $E_{\mathrm{T}}>75 \mathrm{GeV}$. The more stringent choice of $\left|y^{*}\right|<0.3$ selects higher- $p_{\mathrm{T}}$ jets at a given invariant mass and thus provides a mass distribution that is unbiased by the leading-jet selection from $m_{j j}=450 \mathrm{GeV}$.

Background estimation.-The invariant mass spectrum expected from SM dijet production is predicted to be smooth and falling. Prior dijet searches at various collision energies [7,28-32] have found a variety of simple functional forms to describe this shape; however, given the statistical precision of the data and the wide invariant mass range covered by this search, none of the single, simple functional forms can provide a good description of the data.

The SM background distribution is determined using a sliding-window fit [3], where a fitted functional form is evaluated at the bin at the center of a window, which then slides in one-bin steps along the $m_{j j}$ distribution. The evaluated background estimates evaluated in each bin are then collated to form the final background estimate. The signal selection with $\left|y^{*}\right|<0.6$ uses a window size of 19 bins in the $m_{j j}$ spectrum from 531 to $2080 \mathrm{GeV}$, which spans 34 bins in total. The signal selection with $\left|y^{*}\right|<0.3$ uses a window size of 27 bins over a total of 40 bins, in the range $400<m_{j j}<2080 \mathrm{GeV}$. The bin sizes have been chosen according to the simulated invariant mass resolution: $\sigma_{p_{\mathrm{T}}} / p_{\mathrm{T}}=10.6 / p_{\mathrm{T}} \oplus 0.27 / \sqrt{p_{\mathrm{T}}} \oplus 0.039$. The sliding window, however, can not be extended beyond the lower edge of the $m_{j j}$ range used in each signal selection. Therefore, for the first 9 (13) bins in the $\left|y^{*}\right|<0.6$ $\left(\left|y^{*}\right|<0.3\right)$ signal selection, which corresponds to one half of the window size, the window is fixed to the lower edge of the spectrum and instead of evaluating the fitted functional form at the window center, it is evaluated for each bin in turn. For invariant masses higher than the $m_{j j}$ range used for the search, the window is allowed to extend 
beyond the range, to 2970 (3490) $\mathrm{GeV}$ for the $\left|y^{*}\right|<0.6$ (0.3) signal selection, and the fit is evaluated at the center of the window.

In each sliding window, three functional forms are fit to the data: a five-parameter function of the form

$$
f(x)=p_{1}(1-x)^{p_{2}} x^{p_{3}+p_{4} \ln x+p_{5} \ln x^{2}},
$$

where $p_{i}$ are free parameters and $x \equiv m_{j j} / \sqrt{s}$; a fourparameter function, which is the same as Eq. (1) but with $p_{5}=0$; and a four-parameter function used by the UA2 Collaboration [28], defined as

$$
f(x)=\frac{p_{1}}{x^{p_{2}}} \mathrm{e}^{-p_{3} x-p_{4} x^{2}} .
$$

The function used for each signal selection is the one that yields the best $\chi^{2}$ over the full fitted $m_{j j}$ range. An alternative function is chosen to evaluate a systematic uncertainty. For the signal selection with $\left|y^{*}\right|<0.6$, Eq. (1) is used, yielding a $\chi^{2} p$ value of 0.13 , while the alternative function is the four-parameter function with a $\chi^{2}$ $p$ value of 0.11 . For the signal selection with $\left|y^{*}\right|<0.3$, the four-parameter version of Eq. (1) yields the best $\chi^{2} p$ value of 0.42 and the alternative function is Eq. (2), with a $\chi^{2}$ $p$ value of 0.35 .

The size of the sliding window is optimized to yield the best $\chi^{2}$ value for the full $m_{j j}$ range while still being larger than the width of the expected signals and therefore insensitive to potential signal contributions. This latter requirement is checked by including signal models in pseudo-data samples and studying the dependence of the signal sensitivity on different window sizes.

Systematic uncertainties in the estimate of the background used in setting limits include the uncertainty due to the choice of functional form and uncertainties in the fit parameter values. The effect of the choice of functional form is evaluated by comparing the nominal function to the alternative. The uncertainties in the fit parameter values are evaluated using pseudoexperiments, where the pseudodata are drawn from Poisson fluctuations around the nominal background model.

Results and limits.-Figure 4 shows the invariant mass distributions for dijet events in each signal region including the results from the sliding-window background estimates. The $\chi^{2} p$ value of the overall background is 0.13 for the $\left|y^{*}\right|<0.6$ signal selection and 0.42 for the $\left|y^{*}\right|<0.3$ signal selection, indicating the data agrees well with the background estimate. The most discrepant interval identified by the BumpHunter algorithm $[33,34]$ is $889-1007 \mathrm{GeV}$ for events with $\left|y^{*}\right|<0.6$. Accounting for statistical uncertainties only, the probability of observing a deviation at least as significant as that observed in data, anywhere in this distribution, is 0.44 and corresponds to significance of $0.16 \sigma$. Thus, there is no evidence of any localized excess.

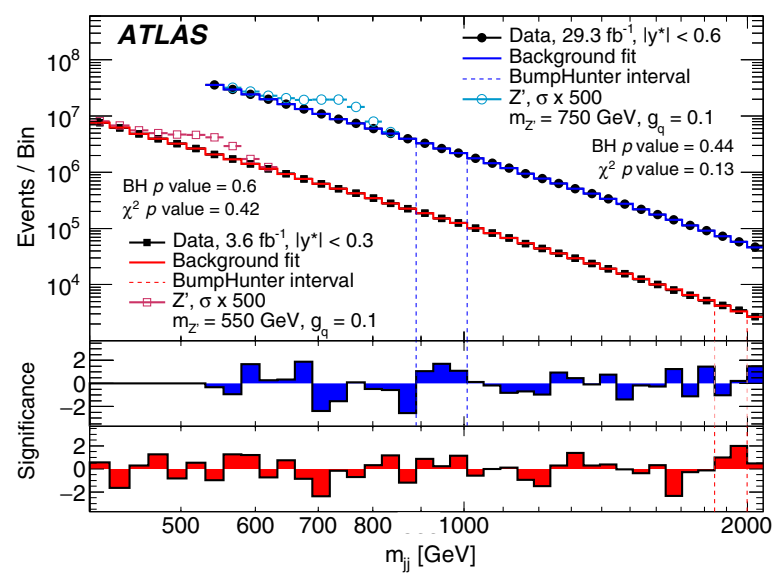

FIG. 4. The reconstructed dijet mass distribution (filled points) for events in the $\left|y^{*}\right|<0.3$ and $\left|y^{*}\right|<0.6$ signal regions. Solid lines depict the background estimate obtained by a slidingwindow fit. Overall agreement between the background estimate and the data is quantified by the $\chi^{2} p$ value. The most discrepant localized excess in either signal region identified by the BumpHunter algorithm is indicated by the vertical lines. The open points show two possible signal models. The lower panels show the bin-by-bin significances of differences between the data and the background estimate, considering only statistical uncertainties (see Ref. [35]).

Limits are set on both a leptophobic $Z^{\prime}$ simplified dark-matter model [36] and a generic Gaussian model. The $Z^{\prime}$ simplified model assumes axial-vector couplings to SM quarks and to a Dirac fermion dark-matter candidate. No interference with the SM is simulated. Signal samples were generated so that the decay rate of the $Z^{\prime}$ into darkmatter particles is negligible and the dijet production rate and resonance width depend only on the coupling of the $Z^{\prime}$ to quarks, $g_{q}$, and the mass of the resonance, $m_{Z^{\prime}}$ [9]. The model's matrix elements were calculated in MADGRAPH 5 [37] and parton showering was performed in PYTHIA 8 [38] with the A14 set of tuned parameters for underlying event [39] and NNPDF2.3 parton distribution functions [40].

The width of a $Z^{\prime}$ resonance with $g_{q}=0.10$, including parton shower and detector resolution effects, is approximately $7 \%$. Limits are set on the cross section, $\sigma$, times acceptance, $A$, times branching ratio, $B$, of the model, and then displayed in the $\left(g_{q}, m_{Z^{\prime}}\right)$ plane [41]. The acceptance for a mass of $550 \mathrm{GeV}$ is $20 \%$ for a $Z^{\prime}$ simplified model with $g_{q}=0.10$ for the $\left|y^{*}\right|<0.3$ signal selection, and $41 \%$ for a signal of mass equal to $750 \mathrm{GeV}$ for the $\left|y^{*}\right|<0.6$ signal selection.

Limits are also set on a generic model where the signal is modeled as a Gaussian contribution to the observed $m_{j j}$ distribution. For a given mean mass, $m_{G}$, four different Gaussian widths are considered: a width equal to the simulated mass resolution (which ranges between $4 \%$ and $6 \%$ ), and the fixed fractions $5 \%, 7 \%$, and $10 \%$ of $m_{G}$. As the width increases, the expected signal contribution is distributed across more bins. Wider signals are 
therefore less affected by statistical fluctuations from the data in a single bin. The results can be used to set limits on models of new phenomena besides that of the $Z^{\prime}$ simplified model and are applicable when the resonance is sufficiently narrow and the parton distribution function and nonperturbative effects can be safely truncated or neglected, as described in Ref. [31]. These criteria are often met if the $m_{j j}$ distribution for a signal approaches a Gaussian distribution after applying the kinematic selection criteria of the resonance analysis, so that $95 \%$ of the signal lies within $20 \%$ of the Gaussian mean mass. Models of new resonances with an intrinsic width much smaller than $5 \%$ of its mass should be compared to the results with a width equal to the experimental resolution. For models with a larger width, the limit that best matches their width should be used. More-detailed instructions can be found in Appendix A of Ref. [31].

A Bayesian method is applied to the data and simulation of the signal models at a series of discrete masses to set 95\% credibility-level upper limits on the cross section times acceptance [30] for the signals described above. The method uses a constant prior for the signal cross section and Gaussian priors for nuisance parameters corresponding to systematic uncertainties. For both observed and expected limits, the sliding window fit is performed for each value of the mass parameter, adding the tested signal shape with a floating normalization to the functional forms stated above. The expected limits are calculated using pseudoexperiments generated from the fit parameters of those functional forms. The uncertainties on the $Z^{\prime}$ signal model include the jet energy scale and the luminosity. The impact of the jet energy resolution uncertainty is negligible. For the Gaussian model, a constant jet energy scale uncertainty of 3\% is applied in accordance with the measured impact of this uncertainty on the $Z^{\prime}$ samples. The uncertainty in the integrated luminosity is $\pm 2.2 \%$, derived following a methodology similar to that detailed in Ref. [42]. The systematic uncertainties in the background estimate include the choice of the fit function and the uncertainty in the fit parameter values, as described above.

Figure 5 shows limits on the coupling to quarks, $g_{q}$, as a function of the mass $m_{Z^{\prime}}$ for the $Z^{\prime}$ model. Figure 6 shows limits on a possible Gaussian contribution with a width equal to the detector resolution as a function of the mean mass, $m_{G}$. In both the $Z^{\prime}$ and Gaussian models, upper limits for masses from 450 to $700 \mathrm{GeV}$ are derived using the distribution with $\left|y^{*}\right|<0.3$, which is sensitive to the lower masses. Limits for masses above $700 \mathrm{GeV}$ are derived from the $m_{j j}$ distribution with $\left|y^{*}\right|<0.6$, except for Gaussian signals with a width of $10 \%$ where only the $\left|y^{*}\right|<0.3$ distribution is used.

The limit results show an upward fluctuation at masses of approximately $1 \mathrm{TeV}$ in the $\left|y^{*}\right|<0.6$ signal region. This is not seen in Fig. 4; when searching for excesses in the data, a background-only hypothesis is used for the sliding

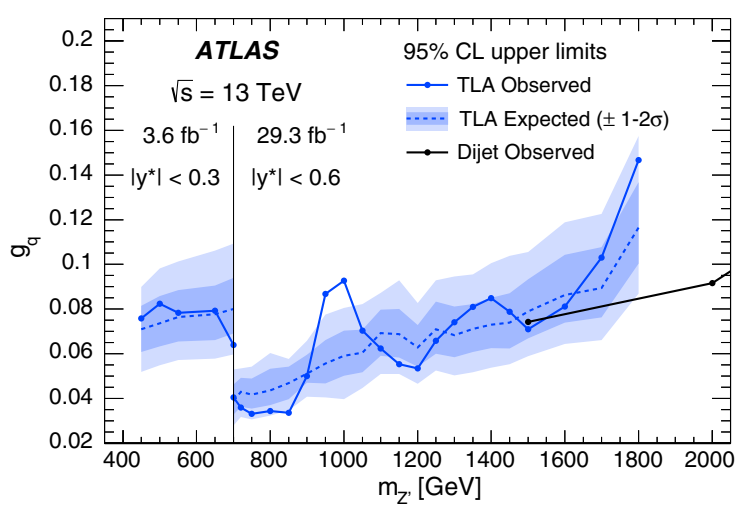

FIG. 5. The $95 \%$ credibility-level observed and expected upper limits on $g_{q}$ as a function of $m_{Z^{\prime}}$ for the $Z^{\prime}$ model described in the text. The lower-mass part of the limits from Ref. [3] is also shown. Couplings above the solid lines are excluded. The solid and dashed lines represent the observed and expected limits, respectively, and are obtained accounting for the scaling of the signal cross section with $g_{q}^{2}$. The different $y^{*}$ selections are described in the text.

window fit. In the observed and expected limits, the fit includes the signal shape in addition to the background parameterization, and can adapt to local data fluctuations that mimic a signal shape. The $\left|y^{*}\right|<0.6$ signal region, which uses a smaller sliding-window size, is especially sensitive to the difference in the two approaches. Therefore,

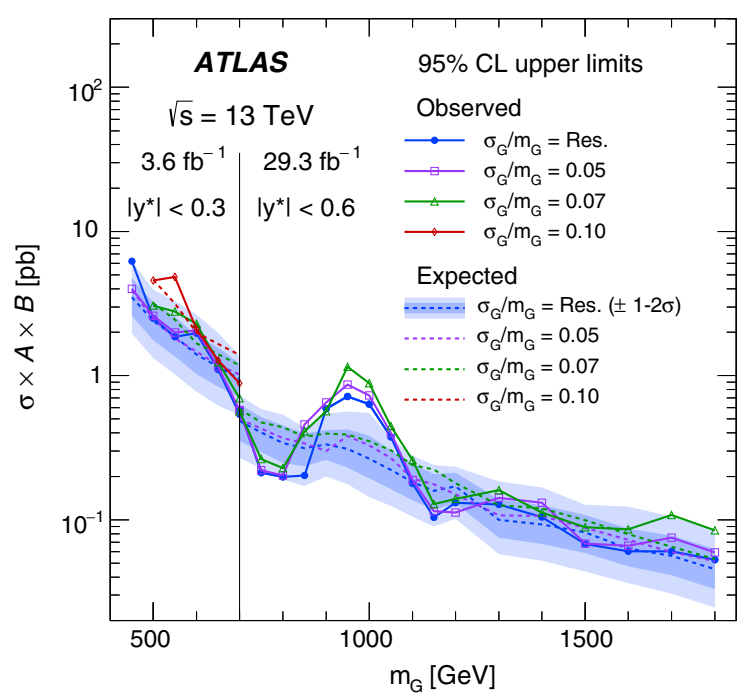

FIG. 6. The 95\% credibility-level observed upper limits on $\sigma \times A \times B$ for two jets for a hypothetical signal producing a Gaussian contribution to the observed $m_{j j}$ distribution. The limits are shown for a relative width $\sigma_{G} / m_{G}$ corresponding to a width equal to the detector mass resolution. While the vertical axis is shared by the two selections, this signal acceptance varies; thus, the two sets of limit points relate to two different interpretations of $\sigma \times A \times B$ (see text for some typical acceptance values used for models considered by this search). The different $y^{*}$ selections are described in the text. 
limits were not set on signals with a width of $10 \%$ for the $\left|y^{*}\right|<0.6$ signal region as the signal is too wide for the sliding-window size.

Conclusions. - In conclusion, this analysis searches for resonances with masses between $450 \mathrm{GeV}$ and $1800 \mathrm{GeV}$ in dijet events using trigger-level jets in $29.3 \mathrm{fb}^{-1}$ of $\sqrt{s}=$ $13 \mathrm{TeV}$ proton-proton collision data recorded by the ATLAS detector at the LHC. The invariant mass distribution presents no significant local excesses compared to the estimated SM background. This analysis provides $95 \%$ credibility-level limits on $Z^{\prime}$ signals and cross sections for new processes that would produce a Gaussian contribution to the dijet mass distribution. Over much of the mass range, the sensitivity to the coupling to quarks, $g_{q}$, is improved by a factor of 2 or more compared to pre-LHC and $\sqrt{s}=8$ and $13 \mathrm{TeV}$ ATLAS results, and is comparable to CMS searches at $\sqrt{s}=8$ and $13 \mathrm{TeV}$ using this technique. Gaussian contributions with effective cross sections times acceptance ranging from approximately $6.5 \mathrm{pb}$ at $450 \mathrm{GeV}$, to $0.4 \mathrm{pb}$ at $700 \mathrm{GeV}$, to $0.05 \mathrm{pb}$ at $1800 \mathrm{GeV}$ are excluded.

We thank CERN for the very successful operation of the LHC, as well as the support staff from our institutions without whom ATLAS could not be operated efficiently. We acknowledge the support of ANPCyT, Argentina; YerPhI, Armenia; ARC, Australia; BMWFW and FWF, Austria; ANAS, Azerbaijan; SSTC, Belarus; CNPq and FAPESP, Brazil; NSERC, NRC and CFI, Canada; CERN; CONICYT, Chile; CAS, MOST and NSFC, China; COLCIENCIAS, Colombia; MSMT CR, MPO CR and VSC CR, Czech Republic; DNRF and DNSRC, Denmark; IN2P3-CNRS, CEA-DRF/IRFU, France; SRNSFG, Georgia; BMBF, HGF, and MPG, Germany; GSRT, Greece; RGC, Hong Kong SAR, China; ISF, I-CORE and Benoziyo Center, Israel; INFN, Italy; MEXT and JSPS, Japan; CNRST, Morocco; NWO, Netherlands; RCN, Norway; MNiSW and NCN, Poland; FCT, Portugal; MNE/IFA, Romania; MES of Russia and NRC KI, Russian Federation; JINR; MESTD, Serbia; MSSR, Slovakia; ARRS and MIZŠ, Slovenia; DST/NRF, South Africa; MINECO, Spain; SRC and Wallenberg Foundation, Sweden; SERI, SNSF and Cantons of Bern and Geneva, Switzerland; MOST, Taiwan; TAEK, Turkey; STFC, United Kingdom; DOE and NSF, United States of America. In addition, individual groups and members have received support from BCKDF, the Canada Council, CANARIE, CRC, Compute Canada, FQRNT, and the Ontario Innovation Trust, Canada; EPLANET, ERC, ERDF, FP7, Horizon 2020 and Marie Skłodowska-Curie Actions, European Union; Investissements d'Avenir Labex and Idex, ANR, Région Auvergne and Fondation Partager le Savoir, France; DFG and AvH Foundation, Germany; Herakleitos, Thales and Aristeia programmes co-financed by EU-ESF and the Greek NSRF; BSF, GIF and Minerva, Israel; BRF, Norway; CERCA Programme Generalitat de Catalunya, Generalitat Valenciana, Spain; the Royal Society and Leverhulme Trust, United Kingdom. The crucial computing support from all WLCG partners is acknowledged gratefully, in particular from CERN, the ATLAS Tier-1 facilities at TRIUMF (Canada), NDGF (Denmark, Norway, Sweden), CC-IN2P3 (France), KIT/GridKA (Germany), INFN-CNAF (Italy), NL-T1 (Netherlands), PIC (Spain), ASGC (Taiwan), RAL (UK) and BNL (USA), the Tier-2 facilities worldwide and large non-WLCG resource providers. Major contributors of computing resources are listed in Ref. [43].

[1] R. M. Harris and K. Kousouris, Searches for dijet resonances at hadron colliders, Int. J. Mod. Phys. A 26, 5005 (2011).

[2] CMS Collaboration, Search for Narrow Resonances Decaying to Dijets in Proton-Proton Collisions at $\sqrt{s}=13 \mathrm{TeV}$, Phys. Rev. Lett. 116, 071801 (2016).

[3] ATLAS Collaboration, Search for new phenomena in dijet events using $37 \mathrm{fb}^{-1}$ of $p p$ collision data collected at $\sqrt{s}=$ $13 \mathrm{TeV}$ with the ATLAS detector, Phys. Rev. D 96, 052004 (2017).

[4] UA1 Collaboration, Angular distributions and structure functions from two jet events at the CERN SPS p anti-p collider, Phys. Lett. B 136, 294 (1984).

[5] UA1 Collaboration, Two jet mass distributions at the CERN proton-antiproton Collider, Phys. Lett. B 209, 127 (1988).

[6] UA2 Collaboration, Measurement of jet production properties at the CERN $p p$ collider, Phys. Lett. B 144, 283 (1984).

[7] CDF Collaboration, Search for new particles decaying into dijets in proton-antiproton collisions at $\sqrt{s}=1.96 \mathrm{TeV}$, Phys. Rev. D 79, 112002 (2009).

[8] B. A. Dobrescu and F. Yu, Coupling-mass mapping of dijet peak searches, Phys. Rev. D 88, 035021 (2013).

[9] M. Chala, F. Kahlhoefer, M. McCullough, G. Nardini, and K. Schmidt-Hoberg, Constraining dark sectors with monojets and dijets, J. High Energy Phys. 07 (2015) 089.

[10] ATLAS Collaboration, Search for new light resonances decaying to jet pairs and produced in association with a photon or a jet in proton-proton collisions at $\sqrt{s}=13 \mathrm{TeV}$ with the ATLAS detector, ATLAS-CONF-2016-070, 2016, https://cds.cern.ch/record/2206221.

[11] CMS Collaboration, Search for low mass vector resonances decaying into quark-antiquark pairs in proton-proton collisions at $\sqrt{s}=13 \mathrm{TeV}$, J. High Energy Phys. 01 (2018) 097.

[12] ATLAS Collaboration, Search for light resonances decaying to boosted quark pairs and produced in association with a photon or a jet in proton-proton collisions at $\sqrt{s}=13 \mathrm{TeV}$ with the ATLAS detector, arXiv:1801.08769.

[13] ATLAS Collaboration, Search for resonances in the mass distribution of jet pairs with one or two jets identified as $b$-jets in proton-proton collisions at $\sqrt{s}=13 \mathrm{TeV}$ with the ATLAS detector, arXiv:1805.09299. 
[14] CMS Collaboration, Search for Narrow Resonances in the b-Tagged Dijet Mass Spectrum in Proton-Proton Collisions at $\sqrt{s}=8 \mathrm{TeV}$, Phys. Rev. Lett. 120, 201801 (2018).

[15] ATLAS Collaboration, Performance of the ATLAS Trigger System in 2015, Eur. Phys. J. C 77, 317 (2017).

[16] CMS Collaboration, Search for Narrow Resonances in Dijet Final States at $\sqrt{s}=8 \mathrm{TeV}$ with the Novel CMS Technique of Data Scouting, Phys. Rev. Lett. 117, 031802 (2016).

[17] CMS Collaboration, Search for narrow and broad dijet resonances in proton-proton collisions at $\sqrt{s}=13 \mathrm{TeV}$ and constraints on dark matter mediators and other new particles, arXiv:1806.00843.

[18] LHCb Collaboration, Measurement of forward $J / \psi$ production cross-sections in $p p$ collisions at $\sqrt{s}=13 \mathrm{TeV}$, J. High Energy Phys. 10 (2015) 172.

[19] ATLAS Collaboration, The ATLAS Experiment at the CERN Large Hadron Collider, J. Instrum. 3, S08003 (2008).

[20] ATLAS uses a right-handed coordinate system with its origin at the nominal interaction point (IP) in the center of the detector and the $z$ axis along the beam pipe. The $x$ axis points from the IP to the center of the LHC ring, and the $y$ axis points upwards. Cylindrical coordinates $(r, \phi)$ are used in the transverse plane, $\phi$ being the azimuthal angle around the $z$ axis. The pseudorapidity is defined in terms of the polar angle $\theta$ as $\eta=-\ln \tan (\theta / 2)$. The rapidity, $y$, is defined as $\frac{1}{2} \ln \left[\left(E+p_{z}\right) /\left(E-p_{z}\right)\right]$, where $E$ denotes the energy of the jet and $p_{z}$ the momentum component of the jet along the beam direction. Angular distance is measured in units of $\Delta R \equiv \sqrt{(\Delta \eta)^{2}+(\Delta \phi)^{2}}$.

[21] M. Cacciari, G. P. Salam, and G. Soyez, The anti- $k_{t}$ jet clustering algorithm, J. High Energy Phys. 04 (2008) 063.

[22] M. Cacciari and G. Salam, Dispelling the $N^{3}$ myth for the $k_{t}$ jet-finder, Phys. Lett. B 641, 57 (2006).

[23] ATLAS Collaboration, Topological cell clustering in the ATLAS calorimeters and its performance in LHC Run 1, Eur. Phys. J. C 77, 490 (2017).

[24] ATLAS Collaboration, Selection of jets produced in $13 \mathrm{TeV}$ proton-proton collisions with the ATLAS detector, CERN Report No. ATLAS-CONF-2015-029, 2015, https://cds .cern.ch/record/2037702.

[25] The trigger-level and offline jets are matched within a radius of $\Delta R=0.4$.

[26] ATLAS Collaboration, Jet energy scale measurements and their systematic uncertainties in proton-proton collisions at $\sqrt{s}=13 \mathrm{TeV}$ with the ATLAS detector, Phys. Rev. D 96, 072002 (2017).

[27] ATLAS Collaboration, Performance of pile-up mitigation techniques for jets in $p p$ collisions at $\sqrt{s}=8 \mathrm{TeV}$ using the ATLAS detector, Eur. Phys. J. C 76, 581 (2016).
[28] UA2 Collaboration, A measurement of two jet decays of the $W$ and $Z$ bosons at the CERN $\bar{p} p$ collider, Z. Phys. C 49, 17 (1991).

[29] CMS Collaboration, Search for resonances in the dijet mass spectrum from $7 \mathrm{TeV} p p$ collisions at CMS, Phys. Lett. B 704, 123 (2011).

[30] ATLAS Collaboration, A search for new physics in dijet mass and angular distributions in $p p$ collisions at $\sqrt{s}=$ $7 \mathrm{TeV}$ measured with the ATLAS detector, New J. Phys. 13, 053044 (2011).

[31] ATLAS Collaboration, Search for new phenomena in the dijet mass distribution using $p p$ collision data at $\sqrt{s}=$ $8 \mathrm{TeV}$ with the ATLAS detector, Phys. Rev. D 91, 052007 (2015).

[32] CMS Collaboration, Search for narrow resonances using the dijet mass spectrum in $p p$ collisions at $\sqrt{s}=8 \mathrm{TeV}$, Phys. Rev. D 87, 114015 (2013).

[33] CDF Collaboration, Global search for new physics with $2.0 \mathrm{fb}^{-1}$ at CDF, Phys. Rev. D 79, 011101 (2009).

[34] G. Choudalakis, On hypothesis testing, trials factor, hypertests and the BumpHunter, arXiv:1101.0390.

[35] G. Choudalakis and D. Casadei, Plotting the differences between data and expectation, Eur. Phys. J. Plus 127, 25 (2012).

[36] D. Abercrombie et al., Dark matter benchmark models for early LHC Run-2 searches: Report of the ATLAS/CMS Dark Matter Forum, arXiv:1507.00966.

[37] J. Alwall, R. Frederix, S. Frixione, V. Hirschi, F. Maltoni, O. Mattelaer, H.-S. Shao, T. Stelzer, P. Torrielli, and M. Zaro, The automated computation of tree-level and next-toleading order differential cross sections, and their matching to parton shower simulations, J. High Energy Phys. 07 (2014) 079.

[38] T. Sjöstrand, S. Mrenna, and P. Skands, A brief introduction to Pythia 8.1, Comput. Phys. Commun. 178, 852 (2008).

[39] ATLAS Collaboration, ATLAS Pythia 8 tunes to $7 \mathrm{TeV}$ data, CERN Report No. ATL-PHYS-PUB-2014-021, 2014, https://cds.cern.ch/record/1966419.

[40] R. D. Ball et al., Parton distributions with LHC data, Nucl. Phys. B867, 244 (2013).

[41] Limits on the coupling are obtained accounting for the scaling of the signal cross section with $g_{q}^{2}$.

[42] ATLAS Collaboration, Luminosity determination in $p p$ collisions at $\sqrt{s}=8 \mathrm{TeV}$ using the ATLAS detector at the LHC, Eur. Phys. J. C 76, 653 (2016).

[43] ATLAS Collaboration, ATLAS Computing Acknowledgements, CERN Report No. ATL-GEN-PUB-2016-002, https://cds.cern.ch/record/2202407.

M. Aaboud, ${ }^{34 d}$ G. Aad, ${ }^{99}$ B. Abbott, ${ }^{124}$ O. Abdinov, ${ }^{13, a}$ B. Abeloos, ${ }^{128}$ S. H. Abidi, ${ }^{165}$ O. S. AbouZeid, ${ }^{143}$ N. L. Abraham, ${ }^{153}$ H. Abramowicz, ${ }^{159}$ H. Abreu, ${ }^{158}$ Y. Abulaiti, ${ }^{6}$ B. S. Acharya,${ }^{64 a, 64 b, b}$ S. Adachi, ${ }^{161}$ L. Adamczyk, ${ }^{81 a}$ J. Adelman, ${ }^{119}$ M. Adersberger, ${ }^{12}$ A. Adiguzel, ${ }^{12 \mathrm{c}}$ T. Adye, ${ }^{141}$ A. A. Affolder, ${ }^{143}$ Y. Afik, ${ }^{158}$ C. Agheorghiesei, ${ }^{27 \mathrm{c}}$ J. A. Aguilar-Saavedra, ${ }^{136 f, 136 a}$ F. Ahmadov, ${ }^{77, c}$ G. Aielli, ${ }^{71 a, 71 b}$ S. Akatsuka, ${ }^{83}$ T. P. A. Åkesson, ${ }^{94}$ E. Akilli, ${ }^{52}$ A. V. Akimov, ${ }^{108}$ G. L. Alberghi, ${ }^{23 b, 23 a}$ J. Albert, ${ }^{174}$ P. Albicocco, ${ }^{49}$ M. J. Alconada Verzini, ${ }^{86}$ S. Alderweireldt, ${ }^{117}$ 
M. Aleksa, ${ }^{35}$ I. N. Aleksandrov, ${ }^{77}$ C. Alexa, ${ }^{27 b}$ G. Alexander ${ }^{159}$ T. Alexopoulos, ${ }^{10}$ M. Alhroob,${ }^{124}$ B. Ali, ${ }^{138}$ G. Alimonti, ${ }^{66 a}$ J. Alison, ${ }^{36}$ S. P. Alkire, ${ }^{145}$ C. Allaire, ${ }^{128}$ B. M. M. Allbrooke, ${ }^{153}$ B. W. Allen, ${ }^{127}$ P. P. Allport, ${ }^{21}$ A. Aloisio,,${ }^{67 a, 67 b}$ A. Alonso,${ }^{39}$ F. Alonso, ${ }^{86}$ C. Alpigiani, ${ }^{145}$ A. A. Alshehri, ${ }^{55}$ M. I. Alstaty,${ }^{99}$ B. Alvarez Gonzalez, ${ }^{35}$ D. Álvarez Piqueras, ${ }^{172}$ M. G. Alviggi, ${ }^{67 a, 67 b}$ B. T. Amadio, ${ }^{18}$ Y. Amaral Coutinho, ${ }^{78 b}$ L. Ambroz, ${ }^{131}$ C. Amelung, ${ }^{26}$ D. Amidei, ${ }^{103}$ S. P. Amor Dos Santos, ${ }^{136 a, 136 c}$ S. Amoroso ${ }^{35}$ C. S. Amrouche, ${ }^{52}$ C. Anastopoulos, ${ }^{146}$ L. S. Ancu, ${ }^{52}$ N. Andari, ${ }^{21}$ T. Andeen, ${ }^{11}$ C. F. Anders, ${ }^{59 b}$ J. K. Anders,${ }^{20}$ K. J. Anderson, ${ }^{36}$ A. Andreazza, ${ }^{66 a, 66 b}$ V. Andrei,${ }^{59 a}$ S. Angelidakis, ${ }^{37}$ I. Angelozzi, ${ }^{118}$ A. Angerami, ${ }^{38}$ A. V. Anisenkov, ${ }^{120 b, 120 a}$ A. Annovi, ${ }^{69 a}$ C. Antel, ${ }^{59 a}$ M. T. Anthony, ${ }^{146}$ M. Antonelli, ${ }^{49}$ D. J. A. Antrim, ${ }^{169}$ F. Anulli, ${ }^{70 a}$ M. Aoki,${ }^{79}$ L. Aperio Bella ${ }^{35}$ G. Arabidze, ${ }^{104}$ Y. Arai, ${ }^{79}$ J. P. Araque, ${ }^{136 a}$ V. Araujo Ferraz, ${ }^{78 b}$ R. Araujo Pereira, ${ }^{78 b}$ A. T. H. Arce, ${ }^{47}$ R. E. Ardell, ${ }^{91}$ F. A. Arduh, ${ }^{86}$ J-F. Arguin, ${ }^{107}$ S. Argyropoulos, ${ }^{75}$ A. J. Armbruster,${ }^{35}$ L. J. Armitage, ${ }^{90}$ O. Arnaez, ${ }^{165}$ H. Arnold, ${ }^{118}$ M. Arratia, ${ }^{31}$ O. Arslan, ${ }^{24}$ A. Artamonov, ${ }^{109, a}$ G. Artoni, ${ }^{131}$ S. Artz,${ }^{97}$

S. Asai, ${ }^{161}$ N. Asbah, ${ }^{44}$ A. Ashkenazi, ${ }^{159}$ E. M. Asimakopoulou, ${ }^{170}$ L. Asquith, ${ }^{153}$ K. Assamagan, ${ }^{29}$ R. Astalos, ${ }^{28 a}$ R. J. Atkin, ${ }^{32 a}$ M. Atkinson, ${ }^{171}$ N. B. Atlay, ${ }^{148}$ K. Augsten, ${ }^{138}$ G. Avolio, ${ }^{35}$ R. Avramidou ${ }^{58 a}$ B. Axen, ${ }^{18}$ M. K. Ayoub, ${ }^{15 a}$ G. Azuelos, ${ }^{107, d}$ A. E. Baas, ${ }^{59 a}$ M. J. Baca, ${ }^{21}$ H. Bachacou, ${ }^{142}$ K. Bachas,${ }^{65 a, 65 b}$ M. Backes, ${ }^{131}$ P. Bagnaia, ${ }^{70 a, 70 b}$ M. Bahmani, ${ }^{82}$ H. Bahrasemani, ${ }^{149}$ A. J. Bailey, ${ }^{172}$ J. T. Baines,${ }^{141}$ M. Bajic,${ }^{39}$ O. K. Baker, ${ }^{181}$ P. J. Bakker, ${ }^{118}$ D. Bakshi Gupta, ${ }^{93}$ E. M. Baldin, ${ }^{120 b, 120 a}$ P. Balek,${ }^{178}$ F. Balli, ${ }^{142}$ W. K. Balunas, ${ }^{133}$ E. Banas, ${ }^{82}$ A. Bandyopadhyay, ${ }^{24}$ S. Banerjee, ${ }^{179, \text { e }}$ A. A. E. Bannoura ${ }^{180}$ L. Barak,${ }^{159}$ W. M. Barbe,${ }^{37}$ E. L. Barberio, ${ }^{102}$ D. Barberis, ${ }^{53 b, 53 a}$ M. Barbero, ${ }^{99}$ T. Barillari, ${ }^{113}$ M-S. Barisits, ${ }^{35}$ J. Barkeloo, ${ }^{127}$ T. Barklow, ${ }^{150}$ N. Barlow, ${ }^{31}$ R. Barnea,${ }^{158}$ S. L. Barnes, ${ }^{58 c}$ B. M. Barnett, ${ }^{141}$ R. M. Barnett, ${ }^{18}$ Z. Barnovska-Blenessy, ${ }^{58 a}$ A. Baroncelli, ${ }^{72 a}$ G. Barone,${ }^{26}$ A. J. Barr, ${ }^{131}$ L. Barranco Navarro, ${ }^{172}$ F. Barreiro, ${ }^{96}$ J. Barreiro Guimarães da Costa, ${ }^{15 a}$ R. Bartoldus, ${ }^{150}$ A. E. Barton,${ }^{87}$ P. Bartos, ${ }^{28 a}$ A. Basalaev, ${ }^{134}$ A. Bassalat, ${ }^{128}$ R. L. Bates, ${ }^{55}$ S. J. Batista, ${ }^{165}$ S. Batlamous, ${ }^{34 \mathrm{e}}$ J. R. Batley, ${ }^{31}$ M. Battaglia, ${ }^{143}$ M. Bauce, ${ }^{70 a, 70 b}$ F. Bauer, ${ }^{142}$ K. T. Bauer, ${ }^{169}$ H. S. Bawa, ${ }^{150, f}$ J. B. Beacham, ${ }^{122}$ M. D. Beattie, ${ }^{87}$ T. Beau, ${ }^{132}$ P. H. Beauchemin, ${ }^{168}$ P. Bechtle, ${ }^{24}$ H. C. Beck, ${ }^{51}$ H. P. Beck, ${ }^{20, \mathrm{~g}}$ K. Becker, ${ }^{50}$ M. Becker, ${ }^{97}$ C. Becot, ${ }^{121}$ A. Beddall, ${ }^{12 \mathrm{~d}}$ A. J. Beddall, ${ }^{12 \mathrm{a}}$ V. A. Bednyakov, ${ }^{77}$ M. Bedognetti, ${ }^{118}$ C. P. Bee, ${ }^{152}$ T. A. Beermann, ${ }^{35}$ M. Begalli, ${ }^{78 b}$ M. Begel, ${ }^{29}$ A. Behera, ${ }^{152}$ J. K. Behr, ${ }^{44}$ A. S. Bell, ${ }^{92}$ G. Bella, ${ }^{159}$ L. Bellagamba, ${ }^{23 \mathrm{~b}}$ A. Bellerive, ${ }^{33}$ M. Bellomo, ${ }^{158}$ K. Belotskiy, ${ }^{110}$ N. L. Belyaev, ${ }^{110}$ O. Benary, ${ }^{159, \mathrm{a}}$ D. Benchekroun, ${ }^{34 \mathrm{a}}$ M. Bender, ${ }^{12}$ N. Benekos, ${ }^{10}$ Y. Benhammou, ${ }^{159}$ E. Benhar Noccioli, ${ }^{181}$ J. Benitez, ${ }^{75}$ D. P. Benjamin, ${ }^{47}$ M. Benoit, ${ }^{52}$ J. R. Bensinger, ${ }^{26}$ S. Bentvelsen, ${ }^{118}$ L. Beresford, ${ }^{131}$ M. Beretta ${ }^{49}$ D. Berge,${ }^{44}$ E. Bergeaas Kuutmann, ${ }^{170}$ N. Berger, ${ }^{5}$ L. J. Bergsten, ${ }^{26}$ J. Beringer, ${ }^{18}$ S. Berlendis,${ }^{56}$ N. R. Bernard, ${ }^{100}$ G. Bernardi, ${ }^{132}$ C. Bernius, ${ }^{150}$ F. U. Bernlochner, ${ }^{24}$ T. Berry, ${ }^{91}$ P. Berta, ${ }^{97}$ C. Bertella, ${ }^{15 a}$ G. Bertoli, ${ }^{43 a, 43 b}$ I. A. Bertram, ${ }^{87}$ G. J. Besjes, ${ }^{39}$ O. Bessidskaia Bylund, ${ }^{43 a, 43 b}$ M. Bessner, ${ }^{44}$ N. Besson, ${ }^{142}$ A. Bethani,${ }^{98}$ S. Bethke, ${ }^{113}$ A. Betti, ${ }^{24}$ A. J. Bevan, ${ }^{90}$ J. Beyer,${ }^{113}$ R. M. B. Bianchi, ${ }^{135}$ O. Biebel, ${ }^{112}$ D. Biedermann, ${ }^{19}$ R. Bielski, ${ }^{98}$ K. Bierwagen, ${ }^{97}$ N. V. Biesuz, ${ }^{69,69 b}$ M. Biglietti, ${ }^{72 a}$ T. R. V. Billoud, ${ }^{107}$ M. Bindi, ${ }^{51}$ A. Bingul, ${ }^{12 \mathrm{~d}}$ C. Bini, ${ }^{70 a, 70 b}$ S. Biondi, ${ }^{23 b, 23 \mathrm{a}}$ T. Bisanz, ${ }^{51}$ J. P. Biswal, ${ }^{159}$ C. Bittrich, ${ }^{46}$ D. M. Bjergaard, ${ }^{47}$ J. E. Black, ${ }^{150}$ K. M. Black, ${ }^{25}$ R. E. Blair, ${ }^{6}$ T. Blazek ${ }^{28 \mathrm{a}}$ I. Bloch, ${ }^{44}$ C. Blocker ${ }^{26}$ A. Blue, ${ }^{55}$ U. Blumenschein, ${ }^{90}$ Blunier, ${ }^{144 a}$ G. J. Bobbink, ${ }^{118}$ V. S. Bobrovnikov, ${ }^{120 b, 120 a}$ S. S. Bocchetta, ${ }^{94}$ A. Bocci, ${ }^{47}$ D. Boerner, ${ }^{180}$ D. Bogavac, ${ }^{112}$

A. G. Bogdanchikov, ${ }^{120 b, 120 a}$ C. Bohm, ${ }^{43 a}$ V. Boisvert, ${ }^{91}$ P. Bokan, ${ }^{170, h}$ T. Bold ${ }^{81 a}$ A. S. Boldyrev, ${ }^{111}$ A. E. Bolz, ${ }^{59 b}$ M. Bomben, ${ }^{132}$ M. Bona,${ }^{90}$ J. S. Bonilla, ${ }^{127}$ M. Boonekamp, ${ }^{142}$ A. Borisov,${ }^{140}$ G. Borissov,${ }^{87}$ J. Bortfeldt, ${ }^{35}$ D. Bortoletto, ${ }^{131}$ V. Bortolotto, ${ }^{71 a, 61 b, 61 c, 71 b}$ D. Boscherini, ${ }^{23 b}$ M. Bosman, ${ }^{14}$ J. D. Bossio Sola, ${ }^{30}$ J. Boudreau, ${ }^{135}$ E. V. Bouhova-Thacker ${ }^{87}$ D. Boumediene, ${ }^{37}$ C. Bourdarios, ${ }^{128}$ S. K. Boutle,${ }^{55}$ A. Boveia, ${ }^{122}$ J. Boyd,${ }^{35}$ I. R. Boyko, ${ }^{77}$ A. J. Bozson, ${ }^{91}$ J. Bracinik, ${ }^{21}$ N. Brahimi, ${ }^{99}$ A. Brandt, ${ }^{8}$ G. Brandt ${ }^{180}$ O. Brandt, ${ }^{59 a}$ F. Braren, ${ }^{44}$ U. Bratzler ${ }^{162}$ B. Brau, ${ }^{100}$ J. E. Brau, ${ }^{127}$ W. D. Breaden Madden, ${ }^{55}$ K. Brendlinger, ${ }^{44}$ A. J. Brennan, ${ }^{102}$ L. Brenner, ${ }^{44}$ R. Brenner, ${ }^{170}$ S. Bressler, ${ }^{178}$ B. Brickwedde,${ }^{97}$ D. L. Briglin, ${ }^{21}$ D. Britton, ${ }^{55}$ D. Britzger, ${ }^{59 b}$ I. Brock, ${ }^{24}$ R. Brock, ${ }^{104}$ G. Brooijmans, ${ }^{38}$ T. Brooks, ${ }^{91}$ W. K. Brooks, ${ }^{144 b}$ E. Brost, ${ }^{119}$ J. H. Broughton, ${ }^{21}$ P. A. Bruckman de Renstrom, ${ }^{82}$ D. Bruncko, ${ }^{28 b}$ A. Bruni, ${ }^{23 b}$ G. Bruni, ${ }^{23 b}$ L. S. Bruni, ${ }^{118}$ S. Bruno, ${ }^{71 a, 71 b}$ B. H. Brunt, ${ }^{31}$ M. Bruschi, ${ }^{23 b}$ N. Bruscino, ${ }^{135}$ P. Bryant,${ }^{36}$ L. Bryngemark, ${ }^{44}$ T. Buanes,${ }^{17}$ Q. Buat,${ }^{35}$ P. Buchholz, ${ }^{148}$ A. G. Buckley, ${ }^{55}$ I. A. Budagov,${ }^{77}$ F. Buehrer, ${ }^{50}$ M. K. Bugge, ${ }^{130}$ O. Bulekov, ${ }^{110}$ D. Bullock, ${ }^{8}$ T. J. Burch, ${ }^{119}$ S. Burdin, ${ }^{88}$ C. D. Burgard, ${ }^{118}$ A. M. Burger, ${ }^{5}$ B. Burghgrave, ${ }^{119}$ K. Burka, ${ }^{82}$ S. Burke, ${ }^{141}$ I. Burmeister, ${ }^{45}$ J. T. P. Burr, ${ }^{131}$ D. Büscher, ${ }^{50}$ V. Büscher, ${ }^{97}$ E. Buschmann, ${ }^{51}$ P. Bussey, ${ }^{55}$ J. M. Butler,${ }^{25}$ C. M. Buttar, ${ }^{55}$ J. M. Butterworth ${ }^{92}$ P. Butti ${ }^{35}$ W. Buttinger, ${ }^{35}$ A. Buzatu, ${ }^{155}$ A. R. Buzykaev, ${ }^{120 b, 120 a}$ G. Cabras, ${ }^{23 b, 23 a}$ S. Cabrera Urbán, ${ }^{172}$ D. Caforio, ${ }^{138}$ H. Cai, ${ }^{171}$ V. M. M. Cairo, ${ }^{2}$ O. Cakir, ${ }^{4 a}$ N. Calace, ${ }^{52}$ P. Calafiura, ${ }^{18}$ A. Calandri, ${ }^{99}$ G. Calderini, ${ }^{132}$ P. Calfayan, ${ }^{63}$ G. Callea, ${ }^{40 b, 40 a}$ L. P. Caloba, ${ }^{78 b}$ S. Calvente Lopez,${ }^{96}$ D. Calvet,${ }^{37}$ S. Calvet, ${ }^{37}$ T. P. Calvet, ${ }^{152}$ M. Calvetti, ${ }^{69 a, 69 b}$ R. Camacho Toro, ${ }^{132}$ S. Camarda, ${ }^{35}$ P. Camarri, ${ }^{71 a, 71 b}$ D. Cameron, ${ }^{130}$ R. Caminal Armadans, ${ }^{100}$ C. Camincher, ${ }^{35}$ S. Campana,${ }^{35}$ M. Campanelli, ${ }^{92}$ 
A. Camplani, ${ }^{66 a, 66 \mathrm{~b}}$ A. Campoverde, ${ }^{148}$ V. Canale,${ }^{67,67 \mathrm{~b}}$ M. Cano Bret, ${ }^{58 \mathrm{c}}$ J. Cantero, ${ }^{125}$ T. Cao, ${ }^{159}$ Y. Cao, ${ }^{171}$ M. D. M. Capeans Garrido, ${ }^{35}$ I. Caprini, ${ }^{27 b}$ M. Caprini, ${ }^{27 b}$ M. Capua, ${ }^{40 b, 40 a}$ R. M. Carbone, ${ }^{38}$ R. Cardarelli, ${ }^{71 a}$ F. C. Cardillo, ${ }^{50}$ I. Carli, ${ }^{139}$ T. Carli, ${ }^{35}$ G. Carlino, ${ }^{67 a}$ B. T. Carlson, ${ }^{135}$ L. Carminati, ${ }^{66 a, 66 b}$ R. M. D. Carney, ${ }^{43 a, 43 b}$ S. Caron, ${ }^{117}$ E. Carquin, ${ }^{144 b}$ S. Carrá, ${ }^{66 a 66 \mathrm{~b}}$ G. D. Carrillo-Montoya ${ }^{35}$ D. Casadei ${ }^{32 \mathrm{~b}}$ M. P. Casado, ${ }^{14, \mathrm{i}}$ A. F. Casha, ${ }^{165}$ M. Casolino, ${ }^{14}$ D. W. Casper, ${ }^{169}$ R. Castelijn, ${ }^{118}$ V. Castillo Gimenez, ${ }^{172}$ N. F. Castro, ${ }^{136 a, 136 e}$ A. Catinaccio, ${ }^{35}$ J. R. Catmore, ${ }^{130}$ A. Cattai, ${ }^{35}$ J. Caudron, ${ }^{24}$ V. Cavaliere, ${ }^{29}$ E. Cavallaro, ${ }^{14}$ D. Cavalli, ${ }^{66 a}$ M. Cavalli-Sforza, ${ }^{14}$ V. Cavasinni, ${ }^{69 a, 69 b}$ E. Celebi, ${ }^{12 b}$ F. Ceradini, ${ }^{72 a, 72 b}$ L. Cerda Alberich, ${ }^{172}$ A. S. Cerqueira, ${ }^{78 a}$ A. Cerri, ${ }^{153}$ L. Cerrito, ${ }^{71 a, 71 b}$ F. Cerutti, ${ }^{18}$ A. Cervelli, ${ }^{23 b, 23 a}$ S. A. Cetin, ${ }^{12 b}$ A. Chafaq ${ }^{34 a}$ D. Chakraborty, ${ }^{119}$ S. K. Chan, ${ }^{57}$ W. S. Chan, ${ }^{118}$ Y. L. Chan, ${ }^{61 a}$ P. Chang, ${ }^{171}$ J. D. Chapman, ${ }^{31}$ D. G. Charlton, ${ }^{21}$ C. C. Chau, ${ }^{33}$ C. A. Chavez Barajas, ${ }^{153}$ S. Che, ${ }^{122}$ A. Chegwidden, ${ }^{104}$ S. Chekanov, ${ }^{6}$ S. V. Chekulaev, ${ }^{166 a}$ G. A. Chelkov, ${ }^{77, j}$ M. A. Chelstowska, ${ }^{35}$ C. Chen, ${ }^{58 a}$ C. H. Chen, ${ }^{76}$ H. Chen, ${ }^{29}$ J. Chen, ${ }^{58 a}$ J. Chen, ${ }^{38}$ S. Chen, ${ }^{133}$ S. J. Chen, ${ }^{15 \mathrm{c}}$ X. Chen, ${ }^{15 \mathrm{~b}, \mathrm{k}}$ Y. Chen,${ }^{80}$ Y-H. Chen, ${ }^{44}$ H. C. Cheng, ${ }^{103}$ H. J. Cheng, ${ }^{15 \mathrm{~d}}$ A. Cheplakov, ${ }^{77}$ E. Cheremushkina, ${ }^{140}$

R. Cherkaoui El Moursli, ${ }^{34 \mathrm{e}}$ E. Cheu, ${ }^{7}$ K. Cheung, ${ }^{62}$ L. Chevalier, ${ }^{142}$ V. Chiarella ${ }^{49}$ G. Chiarelli, ${ }^{69 a}$ G. Chiodini, ${ }^{65 a}$ A. S. Chisholm, ${ }^{35}$ A. Chitan, ${ }^{27 b}$ I. Chiu, ${ }^{161}$ Y. H. Chiu, ${ }^{174}$ M. V. Chizhov, ${ }^{77}$ K. Choi, ${ }^{63}$ A. R. Chomont ${ }^{128}$ S. Chouridou, ${ }^{160}$ Y. S. Chow, ${ }^{118}$ V. Christodoulou, ${ }^{92}$ M. C. Chu, ${ }^{61 a}$ J. Chudoba, ${ }^{137}$ A. J. Chuinard, ${ }^{101}$ J. J. Chwastowski, ${ }^{82}$ L. Chytka, ${ }^{126}$ D. Cinca, ${ }^{45}$ V. Cindro, ${ }^{89}$ I. A. Cioară, ${ }^{24}$ A. Ciocio, ${ }^{18}$ F. Cirotto, ${ }^{67 a, 67 b}$ Z. H. Citron, ${ }^{178}$ M. Citterio, ${ }^{66 a}$ A. Clark,${ }^{52}$ M. R. Clark, ${ }^{38}$ P. J. Clark, ${ }^{48}$ C. Clement, ${ }^{43 a, 43 b}$ Y. Coadou, ${ }^{99}$ M. Cobal,${ }^{64 a, 64 c}$ A. Coccaro, ${ }^{53 b, 53 a}$ J. Cochran, ${ }^{76}$ A. E. C. Coimbra, ${ }^{178}$ L. Colasurdo, ${ }^{117}$ B. Cole,${ }^{38}$ A. P. Colijn, ${ }^{118}$ J. Collot, ${ }^{56}$ P. Conde Muiño, ${ }^{136 a, 136 b}$ E. Coniavitis, ${ }^{50}$ S. H. Connell, ${ }^{32 b}$ I. A. Connelly, ${ }^{98}$ S. Constantinescu, ${ }^{27 b}$ F. Conventi,${ }^{67 a, 1}$ A. M. Cooper-Sarkar, ${ }^{131}$ F. Cormier, ${ }^{173}$ K. J. R. Cormier, ${ }^{165}$ M. Corradi, ${ }^{70 a, 70 b}$ E. E. Corrigan, ${ }^{94}$ F. Corriveau, ${ }^{101, m}$ A. Cortes-Gonzalez, ${ }^{35}$ M. J. Costa, ${ }^{172}$ D. Costanzo, ${ }^{146}$ G. Cottin, ${ }^{31}$ G. Cowan, ${ }^{91}$ B. E. Cox,${ }^{98}$ J. Crane, ${ }^{98}$ K. Cranmer, ${ }^{121}$ S. J. Crawley,${ }^{55}$ R. A. Creager, ${ }^{133}$ G. Cree, ${ }^{33}$ S. Crépé-Renaudin, ${ }^{56}$ F. Crescioli, ${ }^{132}$ M. Cristinziani, ${ }^{24}$ V. Croft,${ }^{121}$ G. Crosetti, ${ }^{40 b, 40 a}$ A. Cueto, ${ }^{96}$

T. Cuhadar Donszelmann, ${ }^{146}$ A. R. Cukierman, ${ }^{150}$ M. Curatolo, ${ }^{49}$ J. Cúth, ${ }^{97}$ S. Czekierda, ${ }^{82}$ P. Czodrowski, ${ }^{35}$ M. J. Da Cunha Sargedas De Sousa,${ }^{58 b, 136 \mathrm{~b}}$ C. Da Via,${ }^{98}$ W. Dabrowski, ${ }^{81 \mathrm{a}}$ T. Dado, ${ }^{28 \mathrm{a}, \mathrm{h}}$ S. Dahbi, ${ }^{34 \mathrm{e}}$ T. Dai, ${ }^{103}$ F. Dallaire, ${ }^{107}$ C. Dallapiccola, ${ }^{100}$ M. Dam, ${ }^{39}$ G. D'amen, ${ }^{23 b, 23 a}$ J. R. Dandoy, ${ }^{133}$ M. F. Daneri, ${ }^{30}$ N. P. Dang, ${ }^{179, e}$ N. D. Dann, ${ }^{98}$ M. Danninger, ${ }^{173}$ V. Dao,${ }^{35}$ G. Darbo,${ }^{53 b}$ S. Darmora,${ }^{8}$ O. Dartsi, ${ }^{5}$ A. Dattagupta,${ }^{127}$ T. Daubney, ${ }^{44}$ S. D' Auria, ${ }^{55}$ W. Davey, ${ }^{24}$ C. David, ${ }^{44}$ T. Davidek, ${ }^{139}$ D. R. Davis, ${ }^{47}$ E. Dawe, ${ }^{102}$ I. Dawson, ${ }^{146}$ K. De ${ }^{8}$ R. De Asmundis, ${ }^{67 a}$ A. De Benedetti, ${ }^{124}$ S. De Castro, ${ }^{23 b, 23 a}$ S. De Cecco, ${ }^{70 a, 70 b}$ N. De Groot, ${ }^{117}$ P. de Jong, ${ }^{118}$ H. De la Torre, ${ }^{104}$ F. De Lorenzi, ${ }^{76}$ A. De Maria, ${ }^{51, n}$ D. De Pedis, ${ }^{70 a}$ A. De Salvo, ${ }^{70 a}$ U. De Sanctis,${ }^{71 a, 71 b}$ A. De Santo ${ }^{153}$ K. De Vasconcelos Corga,${ }^{99}$ J. B. De Vivie De Regie,${ }^{128}$ C. Debenedetti, ${ }^{143}$ D. V. Dedovich, ${ }^{77}$ N. Dehghanian, ${ }^{3}$ M. Del Gaudio, ${ }^{40 b, 40 a}$ J. Del Peso, ${ }^{96}$ D. Delgove, ${ }^{128}$ F. Deliot, ${ }^{142}$ C. M. Delitzsch, ${ }^{7}$ M. Della Pietra, ${ }^{67 a, 67 b}$ D. Della Volpe,${ }^{52}$ A. Dell' Acqua, ${ }^{35}$ L. Dell'Asta, ${ }^{25}$ M. Delmastro, ${ }^{5}$ C. Delporte, ${ }^{128}$ P. A. Delsart, ${ }^{56}$ D. A. DeMarco, ${ }^{165}$ S. Demers ${ }^{181}$ M. Demichev, ${ }^{77}$ S. P. Denisov, ${ }^{140}$ D. Denysiuk, ${ }^{118}$ L. D’Eramo, ${ }^{132}$ D. Derendarz, ${ }^{82}$ J. E. Derkaoui, ${ }^{34 \mathrm{~d}}$ F. Derue, ${ }^{132}$ P. Dervan, ${ }^{88}$ K. Desch,${ }^{24}$ C. Deterre ${ }^{44}$ K. Dette, ${ }^{165}$ M. R. Devesa, ${ }^{30}$ P. O. Deviveiros ${ }^{35}$ A. Dewhurst, ${ }^{141}$ S. Dhaliwal,${ }^{26}$ F. A. Di Bello, ${ }^{52}$ A. Di Ciaccio, ${ }^{71 a, 71 b}$ L. Di Ciaccio, ${ }^{5}$

W. K. Di Clemente, ${ }^{133}$ C. Di Donato,${ }^{67 a 67 b}$ A. Di Girolamo, ${ }^{35}$ B. Di Micco, ${ }^{72 a, 72 b}$ R. Di Nardo,${ }^{35}$ K. F. Di Petrillo, ${ }^{57}$ A. Di Simone, ${ }^{50}$ R. Di Sipio, ${ }^{165}$ D. Di Valentino, ${ }^{33}$ C. Diaconu, ${ }^{99}$ M. Diamond, ${ }^{165}$ F. A. Dias, ${ }^{39}$ T. Dias Do Vale, ${ }^{136 a}$ M. A. Diaz, ${ }^{144 a}$ J. Dickinson, ${ }^{18}$ E. B. Diehl, ${ }^{103}$ J. Dietrich, ${ }^{19}$ S. Díez Cornell, ${ }^{44}$ A. Dimitrievska, ${ }^{18}$ J. Dingfelder, ${ }^{24}$ F. Dittus,${ }^{35}$ F. Djama, ${ }^{99}$ T. Djobava, ${ }^{157 b}$ J. I. Djuvsland, ${ }^{59 \mathrm{a}}$ M. A. B. Do Vale ${ }^{78 \mathrm{c}}$ M. Dobre, ${ }^{27 \mathrm{~b}}$ D. Dodsworth, ${ }^{26}$ C. Doglioni, ${ }^{94}$ J. Dolejsi ${ }^{139}$ Z. Dolezal, ${ }^{139}$ M. Donadelli, ${ }^{78 d}$ J. Donini,${ }^{37}$ A. D'onofrio, ${ }^{90}$ M. D'Onofrio, ${ }^{88}$ J. Dopke, ${ }^{141}$ A. Doria ${ }^{67 a}$ M. T. Dova ${ }^{86}$ A. T. Doyle, ${ }^{55}$ E. Drechsler, ${ }^{51}$ E. Dreyer, ${ }^{149}$ T. Dreyer, ${ }^{51}$ M. Dris,${ }^{10}$ Y. Du, ${ }^{58 b}$ J. Duarte-Campderros, ${ }^{159}$ F. Dubinin, ${ }^{108}$ A. Dubreuil, ${ }^{52}$ E. Duchovni, ${ }^{178}$ G. Duckeck, ${ }^{112}$ A. Ducourthial, ${ }^{132}$ O. A. Ducu, ${ }^{107,0}$ D. Duda, ${ }^{118}$ A. Dudarev, ${ }^{35}$ A. C. Dudder, ${ }^{97}$ E. M. Duffield, ${ }^{18}$ L. Duflot, ${ }^{128}$ M. Dührssen, ${ }^{35}$ C. Dülsen, ${ }^{180}$ M. Dumancic, ${ }^{178}$ A. E. Dumitriu, ${ }^{27 b, p}$ A. K. Duncan, ${ }^{55}$ M. Dunford, ${ }^{59 a}$ A. Duperrin, ${ }^{99}$ H. Duran Yildiz, ${ }^{4 a}$ M. Düren, ${ }^{54}$ A. Durglishvili, ${ }^{157 b}$ D. Duschinger, ${ }^{46}$ B. Dutta, ${ }^{44}$ D. Duvnjak, ${ }^{1}$ M. Dyndal,${ }^{44}$ B. S. Dziedzic, ${ }^{82}$ C. Eckardt,${ }^{44}$ K. M. Ecker, ${ }^{113}$ R. C. Edgar, ${ }^{103}$ T. Eifert, ${ }^{35}$ G. Eigen, ${ }^{17}$

K. Einsweiler, ${ }^{18}$ T. Ekelof, ${ }^{170}$ M. El Kacimi,${ }^{34 \mathrm{c}}$ R. El Kosseifi, ${ }^{99}$ V. Ellajosyula, ${ }^{99}$ M. Ellert, ${ }^{170}$ F. Ellinghaus, ${ }^{180}$ A. A. Elliot, ${ }^{174}$ N. Ellis,${ }^{35}$ J. Elmsheuser, ${ }^{29}$ M. Elsing, ${ }^{35}$ D. Emeliyanov, ${ }^{141}$ Y. Enari, ${ }^{161}$ J. S. Ennis, ${ }^{176}$ M. B. Epland, ${ }^{47}$ J. Erdmann, ${ }^{45}$ A. Ereditato, ${ }^{20}$ S. Errede, ${ }^{171}$ M. Escalier, ${ }^{128}$ C. Escobar, ${ }^{172}$ B. Esposito, ${ }^{49}$ O. Estrada Pastor, ${ }^{172}$ A. I. Etienvre, ${ }^{142}$ E. Etzion, ${ }^{159}$ H. Evans, ${ }^{63}$ A. Ezhilov, ${ }^{134}$ M. Ezzi,${ }^{34 \mathrm{e}}$ F. Fabbri, ${ }^{23 b, 23 a}$ L. Fabbri, ${ }^{23 b, 23 a}$ V. Fabiani, ${ }^{117}$ G. Facini, ${ }^{92}$ R. M. Faisca Rodrigues Pereira, ${ }^{136 a}$ R. M. Fakhrutdinov, ${ }^{140}$ S. Falciano, ${ }^{70 a}$ P. J. Falke, ${ }^{5}$ S. Falke, ${ }^{5}$ J. Faltova, ${ }^{139}$

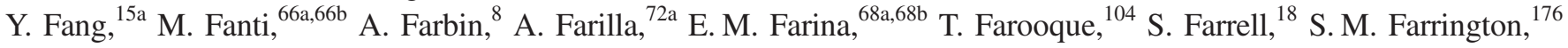


P. Farthouat, ${ }^{35}$ F. Fassi ${ }^{34 \mathrm{e}}$ P. Fassnacht, ${ }^{35}$ D. Fassouliotis, ${ }^{9}$ M. Faucci Giannelli, ${ }^{48}$ A. Favareto, ${ }^{53 b, 53 \mathrm{a}}$ W. J. Fawcett,${ }^{52}$ L. Fayard, ${ }^{128}$ O. L. Fedin, ${ }^{134, q}$ W. Fedorko, ${ }^{173}$ M. Feickert, ${ }^{41}$ S. Feigl, ${ }^{130}$ L. Feligioni, ${ }^{99}$ C. Feng, ${ }^{58 b}$ E. J. Feng, ${ }^{35}$ M. Feng, ${ }^{47}$ M. J. Fenton, ${ }^{55}$ A. B. Fenyuk, ${ }^{140}$ L. Feremenga,${ }^{8}$ J. Ferrando, ${ }^{44}$ A. Ferrari,,${ }^{170}$ P. Ferrari, ${ }^{118}$ R. Ferrari, ${ }^{68 a}$ D. E. Ferreira de Lima, ${ }^{59 b}$ A. Ferrer, ${ }^{172}$ D. Ferrere, ${ }^{52}$ C. Ferretti, ${ }^{103}$ F. Fiedler,${ }^{97}$ A. Filipčič,${ }^{89}$ F. Filthaut,${ }^{117}$ M. Fincke-Keeler, ${ }^{174}$ K. D. Finelli, ${ }^{25}$ M. C. N. Fiolhais, ${ }^{136 a, 136 c, r}$ L. Fiorini, ${ }^{172}$ C. Fischer, ${ }^{14}$ W. C. Fisher,${ }^{104}$ N. Flaschel, ${ }^{44}$ I. Fleck, ${ }^{148}$ P. Fleischmann, ${ }^{103}$ R. R. M. Fletcher, ${ }^{133}$ T. Flick, ${ }^{180}$ B. M. Flierl, ${ }^{112}$ L. M. Flores, ${ }^{133}$ L. R. Flores Castillo, ${ }^{61 a}$ N. Fomin,${ }^{17}$ G. T. Forcolin, ${ }^{98}$ A. Formica, ${ }^{142}$ F. A. Förster, ${ }^{14}$ A. C. Forti, ${ }^{98}$ A. G. Foster, ${ }^{21}$ D. Fournier, ${ }^{128}$ H. Fox,${ }^{87}$ S. Fracchia, ${ }^{146}$ P. Francavilla, ${ }^{69 a, 69 b}$ M. Franchini, ${ }^{23 b, 23 a}$ S. Franchino, ${ }^{59 a}$ D. Francis, ${ }^{35}$ L. Franconi, ${ }^{130}$ M. Franklin, ${ }^{57}$ M. Frate, ${ }^{169}$ M. Fraternali, ${ }^{68 a, 68 b}$ D. Freeborn, ${ }^{92}$ S. M. Fressard-Batraneanu, ${ }^{35}$ B. Freund, ${ }^{107}$ W. S. Freund, ${ }^{78 b}$ D. Froidevaux ${ }^{35}$ J. A. Frost, ${ }^{131}$ C. Fukunaga, ${ }^{162}$ T. Fusayasu, ${ }^{114}$ J. Fuster,${ }^{172}$ O. Gabizon, ${ }^{158}$ A. Gabrielli, ${ }^{23 b, 23 a}$ A. Gabrielli, ${ }^{18}$ G. P. Gach, ${ }^{81 a}$ S. Gadatsch,${ }^{52}$ P. Gadow, ${ }^{113}$ G. Gagliardi, ${ }^{53 b, 53 a}$ L. G. Gagnon, ${ }^{107}$ C. Galea, ${ }^{27 b}$ B. Galhardo, ${ }^{136 a, 136 c}$ E. J. Gallas, ${ }^{131}$ B. J. Gallop, ${ }^{141}$ P. Gallus, ${ }^{138}$ G. Galster,${ }^{39}$ R. Gamboa Goni, ${ }^{90}$ K. K. Gan, ${ }^{122}$ S. Ganguly, ${ }^{178}$ Y. Gao ${ }^{88}$ Y. S. Gao, ${ }^{150, f}$ C. García,${ }^{172}$ J. E. García Navarro, ${ }^{172}$ J. A. García Pascual, ${ }^{15 a}$ M. Garcia-Sciveres ${ }^{18}$ R. W. Gardner, ${ }^{36}$ N. Garelli ${ }^{150}$ V. Garonne, ${ }^{130}$ K. Gasnikova, ${ }^{44}$ A. Gaudiello, ${ }^{53 b, 53 a}$ G. Gaudio, ${ }^{68 a}$ I. L. Gavrilenko, ${ }^{108}$ A. Gavrilyuk,${ }^{109}$ C. Gay, ${ }^{173}$ G. Gaycken, ${ }^{24}$ E. N. Gazis, ${ }^{10}$ C. N. P. Gee,${ }^{141}$ J. Geisen,${ }^{51}$ M. Geisen, ${ }^{97}$ M. P. Geisler,${ }^{59 a}$ K. Gellerstedt, ${ }^{43 a, 43 b}$ C. Gemme, ${ }^{53 b}$ M. H. Genest,${ }^{56}$ C. Geng, ${ }^{103}$ S. Gentile, ${ }^{70 a, 70 b}$ C. Gentsos, ${ }^{160}$ S. George, ${ }^{91}$ D. Gerbaudo, ${ }^{14}$ G. Gessner, ${ }^{45}$ S. Ghasemi, ${ }^{148}$ M. Ghneimat, ${ }^{24}$ B. Giacobbe, ${ }^{23 b}$ S. Giagu, ${ }^{70 a, 70 b}$ N. Giangiacomi, ${ }^{23 b, 23 a}$ P. Giannetti, ${ }^{69 a}$ S. M. Gibson, ${ }^{91}$ M. Gignac, ${ }^{143}$ D. Gillberg, ${ }^{33}$ G. Gilles, ${ }^{180}$ D. M. Gingrich,,${ }^{3, d}$ M. P. Giordani, ${ }^{64 a, 64 c}$ F. M. Giorgi, ${ }^{23 b}$ P. F. Giraud, ${ }^{142}$ P. Giromini, ${ }^{57}$ G. Giugliarelli, ${ }^{64 a, 64 c}$ D. Giugni, ${ }^{66 a}$ F. Giuli, ${ }^{131}$ M. Giulini,${ }^{59 b}$ S. Gkaitatzis, ${ }^{160}$ I. Gkialas, ${ }^{9, s}$ E. L. Gkougkousis, ${ }^{14}$ P. Gkountoumis, ${ }^{10}$ L. K. Gladilin, ${ }^{111}$ C. Glasman, ${ }^{96}$ J. Glatzer, ${ }^{14}$ P. C. F. Glaysher,${ }^{44}$ A. Glazov, ${ }^{44}$ M. Goblirsch-Kolb, ${ }^{26}$ J. Godlewski, ${ }^{82}$ S. Goldfarb, ${ }^{102}$ T. Golling, ${ }^{52}$ D. Golubkov, ${ }^{140}$ A. Gomes, ${ }^{136 a, 136 b, 136 \mathrm{~d}}$

R. Goncalves Gama, ${ }^{78 a}$ R. Gonçalo, ${ }^{136 a}$ G. Gonella ${ }^{50}$ L. Gonella, ${ }^{21}$ A. Gongadze, ${ }^{77}$ F. Gonnella, ${ }^{21}$ J. L. Gonski, ${ }^{57}$ S. González de la Hoz, ${ }^{172}$ S. Gonzalez-Sevilla, ${ }^{52}$ L. Goossens,${ }^{35}$ P. A. Gorbounov, ${ }^{109}$ H. A. Gordon, ${ }^{29}$ B. Gorini, ${ }^{35}$ E. Gorini, ${ }^{65 a, 65 b}$ A. Gorišek, ${ }^{89}$ A. T. Goshaw, ${ }^{47}$ C. Gössling, ${ }^{45}$ M. I. Gostkin, ${ }^{77}$ C. A. Gottardo, ${ }^{24}$ C. R. Goudet, ${ }^{128}$ D. Goujdami, ${ }^{34 \mathrm{c}}$ A. G. Goussiou, ${ }^{145}$ N. Govender, ${ }^{32 b, t}$ C. Goy, ${ }^{5}$ E. Gozani, ${ }^{158}$ I. Grabowska-Bold, ${ }^{81 a}$ P. O. J. Gradin, ${ }^{170}$ E. C. Graham, ${ }^{88}$ J. Gramling, ${ }^{169}$ E. Gramstad, ${ }^{130}$ S. Grancagnolo, ${ }^{19}$ V. Gratchev,${ }^{134}$ P. M. Gravila, ${ }^{27 f}$ C. Gray, ${ }^{55}$ H. M. Gray, ${ }^{18}$ Z. D. Greenwood, ${ }^{93, u}$ C. Grefe, ${ }^{24}$ K. Gregersen, ${ }^{92}$ I. M. Gregor, ${ }^{44}$ P. Grenier, ${ }^{150}$ K. Grevtsov, ${ }^{44}$ J. Griffiths, ${ }^{8}$ A. A. Grillo, ${ }^{143}$ K. Grimm, ${ }^{150}$ S. Grinstein, ${ }^{14, v}$ Ph. Gris,${ }^{37}$ J.-F. Grivaz, ${ }^{128}$ S. Groh,${ }^{97}$ E. Gross,${ }^{178}$ J. Grosse-Knetter, ${ }^{51}$ G. C. Grossi ${ }^{93}$ Z. J. Grout, ${ }^{92}$ C. Grud, ${ }^{103}$ A. Grummer, ${ }^{116}$ L. Guan, ${ }^{103}$ W. Guan, ${ }^{179}$ J. Guenther, ${ }^{35}$ A. Guerguichon, ${ }^{128}$ F. Guescini, ${ }^{166 a}$ D. Guest,${ }^{169}$ R. Gugel,${ }^{50}$ B. Gui, ${ }^{122}$ T. Guillemin, ${ }^{5}$ S. Guindon, ${ }^{35}$ U. Gul,${ }^{55}$ C. Gumpert, ${ }^{35}$ J. Guo, ${ }^{58 c}$ W. Guo,${ }^{103}$ Y. Guo, ${ }^{58 a, w}$ Z. Guo, ${ }^{99}$ R. Gupta, ${ }^{41}$ S. Gurbuz, ${ }^{12 c}$ G. Gustavino, ${ }^{124}$ B. J. Gutelman, ${ }^{158}$ P. Gutierrez, ${ }^{124}$ C. Gutschow, ${ }^{92}$ C. Guyot, ${ }^{142}$ M. P. Guzik, ${ }^{81 a}$ C. Gwenlan, ${ }^{131}$ C. B. Gwilliam, ${ }^{88}$ A. Haas, ${ }^{121}$ C. Haber, ${ }^{18}$ H. K. Hadavand, ${ }^{8}$ N. Haddad,${ }^{34}$ A. Hadef, ${ }^{58 a}$ S. Hageböck, ${ }^{24}$ M. Hagihara, ${ }^{167}$ H. Hakobyan, ${ }^{182, a}$ M. Haleem, ${ }^{175}$ J. Haley, ${ }^{125}$ G. Halladjian, ${ }^{104}$ G. D. Hallewell, ${ }^{99}$ K. Hamacher, ${ }^{180}$ P. Hamal, ${ }^{126}$ K. Hamano,${ }^{174}$ A. Hamilton, ${ }^{32 a}$ G. N. Hamity,${ }^{146}$ K. Han ${ }^{58 a, x}$ L. Han, ${ }^{58 a}$ S. Han, ${ }^{15 d}$ K. Hanagaki, ${ }^{79, y}$ M. Hance, ${ }^{143}$ D. M. Handl, ${ }^{112}$ B. Haney, ${ }^{133}$ R. Hankache, ${ }^{132}$ P. Hanke, ${ }^{59 a}$ E. Hansen,${ }^{94}$ J. B. Hansen, ${ }^{39}$ J. D. Hansen, ${ }^{39}$ M. C. Hansen, ${ }^{24}$ P. H. Hansen, ${ }^{39}$ K. Hara, ${ }^{167}$ A. S. Hard, ${ }^{179}$ T. Harenberg, ${ }^{180}$ S. Harkusha, ${ }^{105}$ P. F. Harrison, ${ }^{176}$ N. M. Hartmann, ${ }^{112}$ Y. Hasegawa, ${ }^{147}$ A. Hasib, ${ }^{48}$ S. Hassani, ${ }^{142}$ S. Haug, ${ }^{20}$ R. Hauser, ${ }^{104}$ L. Hauswald, ${ }^{46}$ L. B. Havener, ${ }^{38}$ M. Havranek, ${ }^{138}$ C. M. Hawkes, ${ }^{21}$ R. J. Hawkings, ${ }^{35}$ D. Hayden,${ }^{104}$ C. Hayes, ${ }^{152}$ C. P. Hays,${ }^{131}$ J. M. Hays, ${ }^{90}$ H. S. Hayward, ${ }^{88}$ S. J. Haywood, ${ }^{141}$ M. P. Heath, ${ }^{48}$ V. Hedberg, ${ }^{94}$ L. Heelan, ${ }^{8}$ S. Heer, ${ }^{24}$ K. K. Heidegger, ${ }^{50}$ J. Heilman, ${ }^{33}$ S. Heim, ${ }^{44}$ T. Heim ${ }^{18}$ B. Heinemann, ${ }^{44, z}$ J. J. Heinrich, ${ }^{112}$ L. Heinrich, ${ }^{121}$ C. Heinz,${ }^{54}$ J. Hejbal, ${ }^{137}$ L. Helary, ${ }^{35}$ A. Held, ${ }^{173}$ S. Hellesund, ${ }^{130}$ S. Hellman, ${ }^{43 a, 43 b}$ C. Helsens,${ }^{35}$ R. C. W. Henderson, ${ }^{87}$ Y. Heng, ${ }^{179}$ S. Henkelmann, ${ }^{173}$

A. M. Henriques Correia, ${ }^{35}$ G. H. Herbert, ${ }^{19}$ H. Herde, ${ }^{26}$ V. Herget, ${ }^{175}$ Y. Hernández Jiménez, ${ }^{32 c}$ H. Herr, ${ }^{97}$ G. Herten, ${ }^{50}$ R. Hertenberger, ${ }^{112}$ L. Hervas, ${ }^{35}$ T. C. Herwig, ${ }^{133}$ G. G. Hesketh, ${ }^{92}$ N. P. Hessey, ${ }^{166 a}$ J. W. Hetherly, ${ }^{41}$ S. Higashino, ${ }^{79}$ E. Higón-Rodriguez, ${ }^{172}$ K. Hildebrand ${ }^{36}$ E. Hill, ${ }^{174}$ J. C. Hill, ${ }^{31}$ K. H. Hiller, ${ }^{44}$ S. J. Hillier, ${ }^{21}$ M. Hils ${ }^{46}$ I. Hinchliffe, ${ }^{18}$ M. Hirose, ${ }^{129}$ D. Hirschbuehl, ${ }^{180}$ B. Hiti, ${ }^{89}$ O. Hladik, ${ }^{137}$ D. R. Hlaluku, ${ }^{32 c}$ X. Hoad ${ }^{48}$ J. Hobbs, ${ }^{152}$ N. Hod, ${ }^{166 a}$ M. C. Hodgkinson, ${ }^{146}$ A. Hoecker ${ }^{35}$ M. R. Hoeferkamp, ${ }^{116}$ F. Hoenig, ${ }^{112}$ D. Hohn, ${ }^{24}$ D. Hohov, ${ }^{128}$ T. R. Holmes, ${ }^{36}$ M. Holzbock, ${ }^{112}$ M. Homann, ${ }^{45}$ S. Honda, ${ }^{167}$ T. Honda ${ }^{79}$ T. M. Hong, ${ }^{135}$ A. Hönle, ${ }^{113}$ B. H. Hooberman,${ }^{171}$ W. H. Hopkins, ${ }^{127}$ Y. Horii, ${ }^{115}$ P. Horn, ${ }^{46}$ A. J. Horton, ${ }^{149}$ L. A. Horyn, ${ }^{36}$ J-Y. Hostachy, ${ }^{56}$ A. Hostiuc,${ }^{145}$ S. Hou, ${ }^{155}$ A. Hoummada, ${ }^{34 a}$ J. Howarth, ${ }^{98}$ J. Hoya ${ }^{86}$ M. Hrabovsky, ${ }^{126}$ J. Hrdinka, ${ }^{35}$ I. Hristova, ${ }^{19}$ J. Hrivnac, ${ }^{128}$ A. Hrynevich, ${ }^{106}$ 
T. Hryn'ova, ${ }^{5}$ P. J. Hsu, ${ }^{62}$ S.-C. Hsu, ${ }^{145}$ Q. Hu, ${ }^{29}$ S. Hu,${ }^{58 c}$ Y. Huang, ${ }^{15 a}$ Z. Hubacek, ${ }^{138}$ F. Hubaut, ${ }^{99}$ M. Huebner, ${ }^{24}$ F. Huegging, ${ }^{24}$ T. B. Huffman, ${ }^{131}$ E. W. Hughes,${ }^{38}$ M. Huhtinen, ${ }^{35}$ R. F. H. Hunter, ${ }^{33}$ P. Huo, ${ }^{152}$ A. M. Hupe, ${ }^{33}$

N. Huseynov, ${ }^{77, c}$ J. Huston, ${ }^{104}$ J. Huth, ${ }^{57}$ R. Hyneman, ${ }^{103}$ G. Iacobucci, ${ }^{52}$ G. Iakovidis, ${ }^{29}$ I. Ibragimov, ${ }^{148}$

L. Iconomidou-Fayard, ${ }^{128}$ Z. Idrissi, ${ }^{34 \mathrm{e}} \mathrm{P}$. Iengo, ${ }^{35} \mathrm{R}$. Ignazzi, ${ }^{39} \mathrm{O}$. Igonkina, ${ }^{118, \text { aa }} \mathrm{R}$. Iguchi, ${ }^{161} \mathrm{~T}$. Iizawa, ${ }^{177}$ Y. Ikegami, ${ }^{79}$ M. Ikeno, ${ }^{79}$ D. Iliadis, ${ }^{160} \mathrm{~N}$. Ilic, ${ }^{150} \mathrm{~F}$. Iltzsche, ${ }^{46} \mathrm{G}$. Introzzi, ${ }^{68,68 \mathrm{~b}} \mathrm{M}$. Iodice, ${ }^{72 \mathrm{a}} \mathrm{K}$. Iordanidou, ${ }^{38} \mathrm{~V}$. Ippolito, ${ }^{70 \mathrm{a}, 70 \mathrm{~b}}$ M. F. Isacson, ${ }^{170} \mathrm{~N}$. Ishijima, ${ }^{129} \mathrm{M}$. Ishino, ${ }^{161} \mathrm{M}$. Ishitsuka, ${ }^{163} \mathrm{C}$. Issever, ${ }^{131} \mathrm{~S}$. Istin, ${ }^{12 c, b b} \mathrm{~F}$. Ito, ${ }^{167} \mathrm{~J}$. M. Iturbe Ponce, ${ }^{61 \mathrm{a}}$ R. Iuppa ${ }^{73 a, 73 b}$ A. Ivina, ${ }^{178}$ H. Iwasaki, ${ }^{79}$ J. M. Izen, ${ }^{42}$ V. Izzo, ${ }^{67 a}$ S. Jabbar, ${ }^{3}$ P. Jacka, ${ }^{137}$ P. Jackson, ${ }^{1}$ R. M. Jacobs, ${ }^{24}$ V. Jain, ${ }^{2}$ G. Jäkel, ${ }^{180}$ K. B. Jakobi, ${ }^{97}$ K. Jakobs, ${ }^{50}$ S. Jakobsen, ${ }^{74}$ T. Jakoubek, ${ }^{137}$ D. O. Jamin, ${ }^{125}$ D. K. Jana, ${ }^{93}$ R. Jansky, ${ }^{52}$ J. Janssen, ${ }^{24}$ M. Janus, ${ }^{51}$ P. A. Janus, ${ }^{81 a}$ G. Jarlskog, ${ }^{94}$ N. Javadov, ${ }^{77, c}$ T. Javůrek, ${ }^{50}$ M. Javurkova, ${ }^{50}$ F. Jeanneau, ${ }^{142}$ L. Jeanty, ${ }^{18}$ J. Jejelava, ${ }^{157 a, c c}$ A. Jelinskas, ${ }^{176}$ P. Jenni, ${ }^{50, d d}$ J. Jeong, ${ }^{44}$ C. Jeske, ${ }^{176}$ S. Jézéquel, ${ }^{5}$ H. Ji, ${ }^{179}$ J. Jia, ${ }^{152}$ H. Jiang, ${ }^{76}$ Y. Jiang, ${ }^{58 \mathrm{a}}$ Z. Jiang, ${ }^{150}$ S. Jiggins, ${ }^{50}$ F. A. Jimenez Morales, ${ }^{37}$ J. Jimenez Pena, ${ }^{172}$ S. Jin, ${ }^{15 c}$ A. Jinaru, ${ }^{27 b}$ O. Jinnouchi, ${ }^{163}$ H. Jivan, ${ }^{32 c}$ P. Johansson, ${ }^{146}$ K. A. Johns, ${ }^{7}$ C. A. Johnson, ${ }^{63}$ W. J. Johnson, ${ }^{145}$ K. Jon-And,${ }^{43 a, 43 b}$ R. W. L. Jones ${ }^{87}$ S. D. Jones, ${ }^{153}$ S. Jones, ${ }^{7}$ T. J. Jones ${ }^{88}$ J. Jongmanns, ${ }^{59 a}$ P. M. Jorge, ${ }^{136 a, 136 b}$ J. Jovicevic, ${ }^{166 a}$ X. Ju, ${ }^{179}$ J. J. Junggeburth, ${ }^{113}$ A. Juste Rozas, ${ }^{14, v}$ A. Kaczmarska, ${ }^{82}$ M. Kado, ${ }^{128}$ H. Kagan, ${ }^{122}$ M. Kagan, ${ }^{150}$ T. Kaji, ${ }^{177}$ E. Kajomovitz, ${ }^{158}$ C. W. Kalderon, ${ }^{94}$ A. Kaluza, ${ }^{97}$ S. Kama, ${ }^{41}$ A. Kamenshchikov, ${ }^{140}$ L. Kanjir, ${ }^{89}$ Y. Kano, ${ }^{161}$ V. A. Kantserov, ${ }^{110}$ J. Kanzaki, ${ }^{79}$ B. Kaplan, ${ }^{121}$ L. S. Kaplan, ${ }^{179}$ D. Kar, ${ }^{32 \mathrm{c}}$ M. J. Kareem, ${ }^{166 \mathrm{~b}}$ E. Karentzos, ${ }^{10}$ S. N. Karpov, ${ }^{77}$ Z. M. Karpova, ${ }^{77}$ V. Kartvelishvili, ${ }^{87}$ A. N. Karyukhin, ${ }^{140}$ K. Kasahara, ${ }^{167}$ L. Kashif,,${ }^{179}$ R. D. Kass, ${ }^{122}$ A. Kastanas, ${ }^{151}$ Y. Kataoka, ${ }^{161}$ C. Kato ${ }^{161}$ J. Katzy ${ }^{44}$ K. Kawade,${ }^{80}$ K. Kawagoe ${ }^{85}$ T. Kawamoto, ${ }^{161}$ G. Kawamura, ${ }^{51}$ E. F. Kay, ${ }^{88}$ V. F. Kazanin, ${ }^{120 b, 120 a}$

R. Keeler, ${ }^{174}$ R. Kehoe, ${ }^{41}$ J. S. Keller, ${ }^{33}$ E. Kellermann, ${ }^{94}$ J. J. Kempster, ${ }^{21}$ J. Kendrick, ${ }^{21}$ O. Kepka, ${ }^{137}$ S. Kersten, ${ }^{180}$

B. P. Kerševan, ${ }^{89}$ R. A. Keyes, ${ }^{101}$ M. Khader, ${ }^{171}$ F. Khalil-Zada, ${ }^{13}$ A. Khanov, ${ }^{125}$ A. G. Kharlamov, ${ }^{120 b, 120 a}$

T. Kharlamova, ${ }^{120 b, 120 a}$ A. Khodinov, ${ }^{164}$ T. J. Khoo, ${ }^{52}$ V. Khovanskiy, ${ }^{109, a}$ E. Khramov, ${ }^{77}$ J. Khubua, ${ }^{157 b}$ S. Kido, ${ }^{80}$ M. Kiehn, ${ }^{52}$ C. R. Kilby, ${ }^{91}$ S. H. Kim, ${ }^{167}$ Y. K. Kim,${ }^{36}$ N. Kimura, ${ }^{64 a, 64 c}$ O. M. Kind, ${ }^{19}$ B. T. King, ${ }^{88}$ D. Kirchmeier, ${ }^{46}$ J. Kirk, ${ }^{141}$ A. E. Kiryunin, ${ }^{113}$ T. Kishimoto, ${ }^{161}$ D. Kisielewska, ${ }^{81 a}$ V. Kitali, ${ }^{44}$ O. Kivernyk, ${ }^{5}$ E. Kladiva, ${ }^{28 b}$

T. Klapdor-Kleingrothaus, ${ }^{50}$ M. H. Klein,${ }^{103}$ M. Klein ${ }^{88}$ U. Klein,${ }^{88}$ K. Kleinknecht, ${ }^{97}$ P. Klimek, ${ }^{119}$ A. Klimentov, ${ }^{29}$ R. Klingenberg, ${ }^{45, a}$ T. Klingl, ${ }^{24}$ T. Klioutchnikova, ${ }^{35}$ F. F. Klitzner, ${ }^{112}$ P. Kluit, ${ }^{118}$ S. Kluth, ${ }^{113}$ E. Kneringer, ${ }^{74}$

E. B. F. G. Knoops, ${ }^{99}$ A. Knue ${ }^{50}$ A. Kobayashi, ${ }^{161}$ D. Kobayashi,${ }^{85}$ T. Kobayashi, ${ }^{161}$ M. Kobel, ${ }^{46}$ M. Kocian, ${ }^{150}$ P. Kodys, ${ }^{139}$ T. Koffas, ${ }^{33}$ E. Koffeman, ${ }^{118}$ N. M. Köhler, ${ }^{113}$ T. Koi, ${ }^{150}$ M. Kolb, ${ }^{59 b}$ I. Koletsou, ${ }^{5}$ T. Kondo, ${ }^{79}$ N. Kondrashova, ${ }^{58 c}$ K. Köneke, ${ }^{50}$ A. C. König, ${ }^{117}$ T. Kono,${ }^{79}$ R. Konoplich,${ }^{121, \text { ee }}$ N. Konstantinidis, ${ }^{92}$ B. Konya, ${ }^{94}$ R. Kopeliansky ${ }^{63}$ S. Koperny, ${ }^{81 a}$ K. Korcyl, ${ }^{82}$ K. Kordas, ${ }^{160}$ A. Korn, ${ }^{92}$ I. Korolkov, ${ }^{14}$ E. V. Korolkova, ${ }^{146}$ O. Kortner, ${ }^{113}$ S. Kortner, ${ }^{113}$ T. Kosek, ${ }^{139}$ V. V. Kostyukhin, ${ }^{24}$ A. Kotwal, ${ }^{47}$ A. Koulouris, ${ }^{10}$ A. Kourkoumeli-Charalampidi, ${ }^{68,68 \mathrm{~b}}$ C. Kourkoumelis,${ }^{9}$ E. Kourlitis, ${ }^{146}$ V. Kouskoura, ${ }^{29}$ A. B. Kowalewska, ${ }^{82}$ R. Kowalewski, ${ }^{174}$ T. Z. Kowalski ${ }^{81 a}$ C. Kozakai, ${ }^{161}$ W. Kozanecki, ${ }^{142}$ A. S. Kozhin, ${ }^{140}$ V. A. Kramarenko, ${ }^{111}$ G. Kramberger ${ }^{89}$ D. Krasnopevtsev, ${ }^{110}$ M. W. Krasny, ${ }^{132}$ A. Krasznahorkay, ${ }^{35}$ D. Krauss, ${ }^{113}$ J. A. Kremer, ${ }^{81 a}$ J. Kretzschmar, ${ }^{88}$ P. Krieger,${ }^{165}$ K. Krizka, ${ }^{18}$ K. Kroeninger, ${ }^{45}$ H. Kroha, ${ }^{113}$ J. Kroll, ${ }^{137}$ J. Kroll, ${ }^{133}$ J. Krstic, ${ }^{16}$ U. Kruchonak, ${ }^{77}$ H. Krüger, ${ }^{24}$ N. Krumnack, ${ }^{76}$ M. C. Kruse, ${ }^{47}$ T. Kubota, ${ }^{102}$ S. Kuday, ${ }^{4 b}$ J. T. Kuechler, ${ }^{180}$ S. Kuehn, ${ }^{35}$ A. Kugel, ${ }^{59 a}$ F. Kuger, ${ }^{175}$ T. Kuhl, ${ }^{44}$ V. Kukhtin, ${ }^{77}$ R. Kukla, ${ }^{99}$ Y. Kulchitsky, ${ }^{105}$ S. Kuleshov, ${ }^{14 \mathrm{~b}}$ Y. P. Kulinich, ${ }^{171}$ M. Kuna, ${ }^{56}$ T. Kunigo, ${ }^{83}$ A. Kupco, ${ }^{137}$ T. Kupfer, ${ }^{45}$ O. Kuprash, ${ }^{159}$ H. Kurashige ${ }^{80}$ L. L. Kurchaninov, ${ }^{166 a}$ Y. A. Kurochkin, ${ }^{105}$ M. G. Kurth, ${ }^{15 d}$ E. S. Kuwertz, ${ }^{174}$ M. Kuze, ${ }^{163}$ J. Kvita, ${ }^{126}$ T. Kwan, ${ }^{174}$ A. La Rosa, ${ }^{113}$ J. L. La Rosa Navarro ${ }^{78 \mathrm{~d}}$ L. La Rotonda, ${ }^{40 \mathrm{~b}, 40 \mathrm{a}}$ F. La Ruffa, ${ }^{40 \mathrm{~b}, 40 \mathrm{a}}$ C. Lacasta, ${ }^{172}$ F. Lacava, ${ }^{70 a}, 70 \mathrm{~b}$ J. Lacey, ${ }^{44}$ D. P. J. Lack, ${ }^{98}$ H. Lacker, ${ }^{19}$ D. Lacour, ${ }^{132}$ E. Ladygin, ${ }^{77}$ R. Lafaye, ${ }^{5}$ B. Laforge, ${ }^{132}$ T. Lagouri, ${ }^{32 \mathrm{c}}$ S. Lai,${ }^{51}$ S. Lammers, ${ }^{63}$ W. Lampl, ${ }^{7}$ E. Lançon, ${ }^{29}$ U. Landgraf,${ }^{50}$ M. P. J. Landon, ${ }^{90}$ M. C. Lanfermann, ${ }^{52}$ V. S. Lang, ${ }^{44}$ J. C. Lange, ${ }^{14}$ R. J. Langenberg, ${ }^{35}$ A. J. Lankford, ${ }^{169}$ F. Lanni, ${ }^{29}$ K. Lantzsch, ${ }^{24}$ A. Lanza, ${ }^{68 a}$ A. Lapertosa, ${ }^{53 b, 53 a}$ S. Laplace, ${ }^{132}$ J. F. Laporte, ${ }^{142}$ T. Lari, ${ }^{66 a}$ F. Lasagni Manghi, ${ }^{23 b, 23 a}$ M. Lassnig, ${ }^{35}$ T. S. Lau, ${ }^{61 a}$ A. Laudrain, ${ }^{128}$ A. T. Law, ${ }^{143}$ P. Laycock, ${ }^{88}$ M. Lazzaroni, ${ }^{66 a, 66 b}$ B. Le, ${ }^{102}$ O. Le Dortz, ${ }^{132}$ E. Le Guirriec, ${ }^{99}$ E. P. Le Quilleuc, ${ }^{142}$ M. LeBlanc, ${ }^{7}$ T. LeCompte, ${ }^{6}$ F. Ledroit-Guillon, ${ }^{56}$ C. A. Lee, ${ }^{29}$ G. R. Lee, ${ }^{144 a}$ L. Lee,${ }^{57}$ S. C. Lee, ${ }^{155}$ B. Lefebvre, ${ }^{101}$ M. Lefebvre ${ }^{174}$ F. Legger, ${ }^{112}$ C. Leggett, ${ }^{18}$ G. Lehmann Miotto, ${ }^{35}$ W. A. Leight, ${ }^{44}$ A. Leisos, ${ }^{160, f f}$ M. A. L. Leite, ${ }^{78 d}$ R. Leitner, ${ }^{139}$ D. Lellouch, ${ }^{178}$ B. Lemmer, ${ }^{51}$ K. J. C. Leney,${ }^{92}$ T. Lenz,${ }^{24}$ B. Lenzi,${ }^{35}$ R. Leone, ${ }^{7}$ S. Leone,${ }^{69 a}$ C. Leonidopoulos, ${ }^{48}$ G. Lerner,${ }^{153}$ C. Leroy, ${ }^{107}$ R. Les, ${ }^{165}$ A. A. J. Lesage, ${ }^{142}$ C. G. Lester ${ }^{31}$ M. Levchenko, ${ }^{134}$ J. Levêque, ${ }^{5}$ D. Levin, ${ }^{103}$ L. J. Levinson, ${ }^{178}$ D. Lewis, ${ }^{90}$

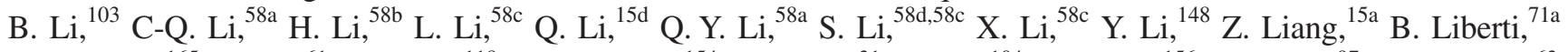
A. Liblong, ${ }^{165}$ K. Lie, ${ }^{61 \mathrm{c}}$ S. Liem, ${ }^{118}$ A. Limosani, ${ }^{154}$ C. Y. Lin, ${ }^{31}$ K. Lin, ${ }^{104}$ S. C. Lin, ${ }^{156}$ T. H. Lin, ${ }^{97}$ R. A. Linck, ${ }^{63}$ 
B. E. Lindquist, ${ }^{152}$ A. L. Lionti, ${ }^{52}$ E. Lipeles, ${ }^{133}$ A. Lipniacka, ${ }^{17}$ M. Lisovyi, ${ }^{59 b}$ T. M. Liss, ${ }^{171, g g}$ A. Lister, ${ }^{173}$ A. M. Litke, ${ }^{143}$ J. D. Little, ${ }^{8}$ B. Liu, ${ }^{76}$ B. L. Liu, ${ }^{6}$ H. B. Liu, ${ }^{29}$ H. Liu, ${ }^{103}$ J. B. Liu, ${ }^{58 a}$ J. K. K. Liu, ${ }^{131}$ K. Liu, ${ }^{132}$ M. Liu, ${ }^{58 a}$ P. Liu, ${ }^{18}$ Y. L. Liu, ${ }^{58 \mathrm{a}}$ Y. W. Liu, ${ }^{58 \mathrm{a}}$ M. Livan, ${ }^{68 \mathrm{a}, 68 \mathrm{~b}}$ A. Lleres, ${ }^{56}$ J. Llorente Merino, ${ }^{15 \mathrm{a}}$ S. L. Lloyd,${ }^{90}$ C. Y. Lo,${ }^{61 b}$ F. Lo Sterzo, ${ }^{41}$ E. M. Lobodzinska, ${ }^{44}$ P. Loch, ${ }^{7}$ F. K. Loebinger ${ }^{98}$ A. Loesle,${ }^{50}$ K. M. Loew, ${ }^{26}$ T. Lohse, ${ }^{19}$ K. Lohwasser,${ }^{146}$ M. Lokajicek, ${ }^{137}$ B. A. Long, ${ }^{25}$ J. D. Long, ${ }^{171}$ R. E. Long, ${ }^{87}$ L. Longo, ${ }^{65 a, 65 b}$ K. A. Looper ${ }^{122}$ J. A. Lopez, ${ }^{144 b}$ I. Lopez Paz, ${ }^{14}$

A. Lopez Solis, ${ }^{132}$ J. Lorenz, ${ }^{112}$ N. Lorenzo Martinez, ${ }^{5}$ M. Losada, ${ }^{22}$ P. J. Lösel, ${ }^{112}$ X. Lou ${ }^{44}$ X. Lou, ${ }^{15 a}$ A. Lounis, ${ }^{128}$ J. Love ${ }^{6}$ P. A. Love, ${ }^{87}$ J. J. Lozano Bahilo, ${ }^{172}$ H. Lu,${ }^{61 a}$ N. Lu, ${ }^{103}$ Y. J. Lu, ${ }^{62}$ H. J. Lubatti, ${ }^{145}$ C. Luci, ${ }^{70 a, 70 b}$ A. Lucotte,${ }^{56}$ C. Luedtke, ${ }^{50}$ F. Luehring, ${ }^{63}$ I. Luise,${ }^{132}$ W. Lukas, ${ }^{74}$ L. Luminari ${ }^{70 a}$ B. Lund-Jensen, ${ }^{151}$ M. S. Lutz, ${ }^{100}$ P. M. Luzi,${ }^{132}$ D. Lynn, ${ }^{29}$ R. Lysak ${ }^{137}$ E. Lytken, ${ }^{94}$ F. Lyu, ${ }^{15 \mathrm{a}}$ V. Lyubushkin, ${ }^{77}$ H. Ma ${ }^{29}$ L. L. Ma ${ }^{58 b}$ Y. Ma ${ }^{58 b}$ G. Maccarrone, ${ }^{49}$ A. Macchiolo, ${ }^{113}$ C. M. Macdonald, ${ }^{146}$ J. Machado Miguens, ${ }^{133,136 \mathrm{~b}}$ D. Madaffari, ${ }^{172}$ R. Madar, ${ }^{37}$ W. F. Mader ${ }^{46}$ A. Madsen, ${ }^{44}$ N. Madysa,${ }^{46}$ J. Maeda,${ }^{80}$ S. Maeland ${ }^{17}$ T. Maeno, ${ }^{29}$ A. S. Maevskiy, ${ }^{111}$ V. Magerl, ${ }^{50}$ C. Maidantchik, ${ }^{78 b}$ T. Maier, ${ }^{12}$ A. Maio, ${ }^{136 a, 136 b, 136 \mathrm{~d}}$ O. Majersky, ${ }^{28 a}$ S. Majewski, ${ }^{127}$ Y. Makida ${ }^{79}$ N. Makovec, ${ }^{128}$ B. Malaescu, ${ }^{132}$ Pa. Malecki ${ }^{82}$ V. P. Maleev ${ }^{134}$ F. Malek,${ }^{56}$ U. Mallik, ${ }^{75}$ D. Malon, ${ }^{6}$ C. Malone,${ }^{31}$ S. Maltezos, ${ }^{10}$ S. Malyukov,${ }^{35}$ J. Mamuzic, ${ }^{172}$ G. Mancini, ${ }^{49}$ I. Mandic ${ }^{89}$ J. Maneira, ${ }^{136 a}$ L. Manhaes de Andrade Filho, ${ }^{78 a}$ J. Manjarres Ramos ${ }^{46}$ K. H. Mankinen, ${ }^{94}$ A. Mann, ${ }^{112}$ A. Manousos, ${ }^{74}$ B. Mansoulie, ${ }^{142}$ J. D. Mansour, ${ }^{15 a}$ M. Mantoani, ${ }^{51}$ S. Manzoni, ${ }^{66 a, 66 b}$ G. Marceca, ${ }^{30}$ L. March, ${ }^{52}$ L. Marchese, ${ }^{131}$ G. Marchiori, ${ }^{132}$ M. Marcisovsky, ${ }^{137}$ C. A. Marin Tobon, ${ }^{35}$ M. Marjanovic, ${ }^{37}$

D. E. Marley ${ }^{103}$ F. Marroquim, ${ }^{78 b}$ Z. Marshall ${ }^{18}$ M. U. F. Martensson, ${ }^{170}$ S. Marti-Garcia, ${ }^{172}$ C. B. Martin, ${ }^{122}$ T. A. Martin, ${ }^{176}$ V. J. Martin, ${ }^{48}$ B. Martin dit Latour, ${ }^{17}$ M. Martinez, ${ }^{14, v}$ V. I. Martinez Outschoorn, ${ }^{100}$ S. Martin-Haugh, ${ }^{141}$ V. S. Martoiu, ${ }^{27 b}$ A. C. Martyniuk, ${ }^{92}$ A. Marzin, ${ }^{35}$ L. Masetti, ${ }^{97}$ T. Mashimo, ${ }^{161}$ R. Mashinistov, ${ }^{108}$ J. Masik, ${ }^{98}$ A. L. Maslennikov, ${ }^{120 b, 120 \mathrm{a}}$ L. H. Mason, ${ }^{102}$ L. Massa, ${ }^{71 \mathrm{a}, 71 \mathrm{~b}}$ P. Mastrandrea, ${ }^{5}$ A. Mastroberardino, ${ }^{40 \mathrm{~b}, 40 \mathrm{a}}$ T. Masubuchi, ${ }^{161}$ P. Mättig, ${ }^{180}$ J. Maurer, ${ }^{27 b}$ B. Maček, ${ }^{89}$ S. J. Maxfield,${ }^{88}$ D. A. Maximov, ${ }^{120 b, 120 a}$ R. Mazini, ${ }^{155}$ I. Maznas, ${ }^{160}$ S. M. Mazza, ${ }^{143}$

N. C. Mc Fadden, ${ }^{116}$ G. Mc Goldrick, ${ }^{165}$ S. P. Mc Kee, ${ }^{103}$ A. McCarn, ${ }^{103}$ T. G. McCarthy, ${ }^{113}$ L. I. McClymont,${ }^{92}$

E. F. McDonald, ${ }^{102}$ J. A. Mcfayden,${ }^{35}$ G. Mchedlidze, ${ }^{51}$ M. A. McKay, ${ }^{41}$ K. D. McLean ${ }^{174}$ S. J. McMahon, ${ }^{141}$ P. C. McNamara, ${ }^{102}$ C. J. McNicol, ${ }^{176}$ R. A. McPherson, ${ }^{174, \mathrm{~m}}$ J. E. Mdhluli, ${ }^{32 \mathrm{c}}$ Z. A. Meadows, ${ }^{100} \mathrm{~S}$. Meehan,${ }^{145}$ T. Megy, ${ }^{50}$ S. Mehlhase, ${ }^{112}$ A. Mehta, ${ }^{88}$ T. Meideck, ${ }^{56}$ B. Meirose, ${ }^{42}$ D. Melini, ${ }^{172, \text { hh }}$ B. R. Mellado Garcia, ${ }^{32 \mathrm{c}}$ J. D. Mellenthin, ${ }^{51}$ M. Melo, ${ }^{28 a}$ F. Meloni, ${ }^{20}$ A. Melzer, ${ }^{24}$ S. B. Menary ${ }^{98}$ L. Meng, ${ }^{88}$ X. T. Meng, ${ }^{103}$ A. Mengarelli, ${ }^{23 b, 23 a}$ S. Menke, ${ }^{113}$ E. Meoni, ${ }^{40 b, 40 a}$ S. Mergelmeyer, ${ }^{19}$ C. Merlassino, ${ }^{20}$ P. Mermod ${ }^{52}$ L. Merola,${ }^{67 a 67 b}$ C. Meroni, ${ }^{66 a}$ F. S. Merritt, ${ }^{36}$ A. Messina, ${ }^{70 a, 70 b}$ J. Metcalfe, ${ }^{6}$ A. S. Mete,${ }^{169}$ C. Meyer, ${ }^{133}$ J. Meyer, ${ }^{158}$ J-P. Meyer, ${ }^{142}$ H. Meyer Zu Theenhausen, ${ }^{59 a}$ F. Miano ${ }^{153}$ R. P. Middleton, ${ }^{141}$ L. Mijovici,${ }^{48}$ G. Mikenberg, ${ }^{178}$ M. Mikestikova, ${ }^{137}$ M. Mikuž,${ }^{89}$ M. Milesi,${ }^{102}$ A. Milic, ${ }^{165}$ D. A. Millar, ${ }^{90}$ D. W. Miller, ${ }^{36}$ A. Milov, ${ }^{178}$ D. A. Milstead ${ }^{43 a, 43 b}$ A. A. Minaenko, ${ }^{140}$ I. A. Minashvili, ${ }^{157 b}$ A. I. Mincer, ${ }^{121}$ B. Mindur, ${ }^{81 a}$ M. Mineev, ${ }^{77}$ Y. Minegishi, ${ }^{161}$ Y. Ming, ${ }^{179}$ L. M. Mir, ${ }^{14}$ A. Mirto, ${ }^{65 a, 65 b}$ K. P. Mistry, ${ }^{133}$ T. Mitani, ${ }^{177}$ J. Mitrevski, ${ }^{112}$ V. A. Mitsou, ${ }^{172}$ A. Miucci, ${ }^{20}$ P. S. Miyagawa, ${ }^{146}$ A. Mizukami ${ }^{79}$ J. U. Mjörnmark, ${ }^{94}$ T. Mkrtchyan, ${ }^{182}$ M. Mlynarikova, ${ }^{139}$ T. Moa, ${ }^{43 a, 43 b}$ K. Mochizuki, ${ }^{107}$ P. Mogg ${ }^{50}$ S. Mohapatra ${ }^{38}$ S. Molander ${ }^{43 a, 43 b}$ R. Moles-Valls, ${ }^{24}$ M. C. Mondragon, ${ }^{104}$ K. Mönig, ${ }^{44}$ J. Monk, ${ }^{39}$ E. Monnier, ${ }^{99}$ A. Montalbano, ${ }^{149}$ J. Montejo Berlingen, ${ }^{35}$ F. Monticelli, ${ }^{86}$ S. Monzani, ${ }^{66 \mathrm{a}}$ R. W. Moore, ${ }^{3}$ N. Morange, ${ }^{128}$ D. Moreno, ${ }^{22}$ M. Moreno Llácer, ${ }^{35}$ P. Morettini, ${ }^{53 \mathrm{~b}}$ M. Morgenstern, ${ }^{118}$ S. Morgenstern, ${ }^{35}$ D. Mori, ${ }^{149}$ T. Mori, ${ }^{161}$ M. Morii, ${ }^{57}$ M. Morinaga, ${ }^{177}$ V. Morisbak, ${ }^{130}$ A. K. Morley, ${ }^{35}$ G. Mornacchi, ${ }^{35}$ J. D. Morris, ${ }^{90}$ L. Morvaj, ${ }^{152}$ P. Moschovakos, ${ }^{10}$ M. Mosidze, ${ }^{157 b}$ H. J. Moss, ${ }^{146}$ J. Moss, ${ }^{150, \text { ii }}$ K. Motohashi,,${ }^{163}$ R. Mount, ${ }^{150}$ E. Mountricha, ${ }^{29}$ E. J. W. Moyse, ${ }^{100}$ S. Muanza, ${ }^{99}$ F. Mueller, ${ }^{113}$ J. Mueller, ${ }^{135}$ R. S. P. Mueller, ${ }^{112}$ D. Muenstermann, ${ }^{87}$ P. Mullen,${ }^{55}$ G. A. Mullier, ${ }^{20}$ F. J. Munoz Sanchez, ${ }^{98}$ P. Murin, ${ }^{28 b}$ W. J. Murray, ${ }^{176,141}$ A. Murrone,${ }^{66 a, 66 b}$ M. Muškinja ${ }^{89}$

C. Mwewa ${ }^{32 a}$ A. G. Myagkov, ${ }^{140, j \mathrm{j}}$ J. Myers, ${ }^{127}$ M. Myska, ${ }^{138}$ B. P. Nachman, ${ }^{18}$ O. Nackenhorst, ${ }^{45}$ K. Nagai,${ }^{131}$

R. Nagai, ${ }^{79, k \mathrm{kk}}$ K. Nagano, ${ }^{79}$ Y. Nagasaka ${ }^{60}$ K. Nagata, ${ }^{167}$ M. Nagel,${ }^{50}$ E. Nagy, ${ }^{99}$ A. M. Nairz, ${ }^{35}$ Y. Nakahama, ${ }^{115}$ K. Nakamura, ${ }^{79}$ T. Nakamura, ${ }^{161}$ I. Nakano, ${ }^{123}$ F. Napolitano, ${ }^{59 a}$ R. F. Naranjo Garcia, ${ }^{44}$ R. Narayan, ${ }^{11}$ D. I. Narrias Villar, ${ }^{59 a}$ I. Naryshkin, ${ }^{134}$ T. Naumann, ${ }^{44}$ G. Navarro, ${ }^{22}$ R. Nayyar, ${ }^{7}$ H. A. Neal, ${ }^{103}$ P. Y. Nechaeva, ${ }^{108}$ T. J. Neep, ${ }^{142}$ A. Negri, ${ }^{68 a, 68 b}$ M. Negrini, ${ }^{23 b}$ S. Nektarijevic, ${ }^{117}$ C. Nellist,${ }^{51}$ M. E. Nelson, ${ }^{131}$ S. Nemecek, ${ }^{137}$ P. Nemethy, ${ }^{121}$ M. Nessi, ${ }^{35,11}$ M. S. Neubauer, ${ }^{171}$ M. Neumann, ${ }^{180}$ P. R. Newman, ${ }^{21}$ T. Y. Ng, ${ }^{61 \mathrm{c}}$ Y. S. Ng, ${ }^{19}$ H. D. N. Nguyen, ${ }^{99}$ T. Nguyen Manh, ${ }^{107}$ E. Nibigira, ${ }^{37}$ R. B. Nickerson, ${ }^{131}$ R. Nicolaidou, ${ }^{142}$ J. Nielsen, ${ }^{143}$ N. Nikiforou, ${ }^{11}$ V. Nikolaenko, ${ }^{140, j j}$ I. Nikolic-Audit, ${ }^{132}$ K. Nikolopoulos,${ }^{21}$ P. Nilsson, ${ }^{29}$ H. R. Nindhito, ${ }^{52}$ Y. Ninomiya,${ }^{79}$ A. Nisati, ${ }^{70 a}$ N. Nishu, ${ }^{58 c}$ R. Nisius, ${ }^{113}$ I. Nitsche,${ }^{45}$ T. Nitta, ${ }^{177}$ T. Nobe, ${ }^{161}$ Y. Noguchi, ${ }^{83}$ M. Nomachi, ${ }^{129}$ I. Nomidis,${ }^{33}$ M. A. Nomura, ${ }^{29}$ T. Nooney, ${ }^{90}$ M. Nordberg, ${ }^{35}$ N. Norjoharuddeen, ${ }^{131}$ T. Novak, ${ }^{89}$ O. Novgorodova, ${ }^{46}$ R. Novotny, ${ }^{138}$ M. Nozaki, ${ }^{79}$ L. Nozka, ${ }^{126}$ K. Ntekas, ${ }^{169}$ E. Nurse, ${ }^{92}$ 
F. Nuti, ${ }^{102}$ F. G. Oakham, ${ }^{33, \mathrm{~d}}$ H. Oberlack, ${ }^{113}$ T. Obermann, ${ }^{24}$ J. Ocariz, ${ }^{132}$ A. Ochi ${ }^{80}$ I. Ochoa ${ }^{38}$ J.P. Ochoa-Ricoux, ${ }^{144 a}$ K. O'Connor, ${ }^{26}$ S. Oda ${ }^{85}$ S. Odaka, ${ }^{79}$ A. Oh, ${ }^{98}$ S. H. Oh, ${ }^{47}$ C. C. Ohm,${ }^{151}$ H. Oide,${ }^{53 b, 53 a}$ H. Okawa, ${ }^{167}$ Y. Okazaki, ${ }^{83}$ Y. Okumura, ${ }^{161}$ T. Okuyama, ${ }^{79}$ A. Olariu, ${ }^{27 b}$ L. F. Oleiro Seabra, ${ }^{136 a}$ S. A. Olivares Pino, ${ }^{144 a}$ D. Oliveira Damazio, ${ }^{29}$ J. L. Oliver, ${ }^{1}$ M. J. R. Olsson, ${ }^{36}$ A. Olszewski, ${ }^{82}$ J. Olszowska, ${ }^{82}$ D. C. O’Neil, ${ }^{149}$ A. Onofre, ${ }^{136 a, 136 e}$ K. Onogi, ${ }^{115}$ P. U.E. Onyisi, ${ }^{11}$ H. Oppen, ${ }^{130}$ M. J. Oreglia, ${ }^{36}$ Y. Oren, ${ }^{159}$ D. Orestano, ${ }^{72 a, 72 b}$ E. C. Orgill, ${ }^{98}$ N. Orlando, ${ }^{61 b}$ A. A. O’Rourke, ${ }^{44}$ R. S. Orr, ${ }^{165}$ B. Osculati,${ }^{53 b, 53 a, a}$ V. O'Shea, ${ }^{55}$ R. Ospanov, ${ }^{58 a}$ G. Otero y Garzon,${ }^{30}$ H. Otono, ${ }^{85}$ M. Ouchrif, ${ }^{34 \mathrm{~d}}$ F. Ould-Saada, ${ }^{130}$ A. Ouraou, ${ }^{142}$ Q. Ouyang, ${ }^{15 a}$ M. Owen, ${ }^{55}$ R. E. Owen, ${ }^{21}$ V. E. Ozcan, ${ }^{12 \mathrm{c}}$ N. Ozturk, ${ }^{8}$ J. Pacalt, ${ }^{126}$ H. A. Pacey, ${ }^{31}$ K. Pachal,${ }^{149}$ A. Pacheco Pages,${ }^{14}$ L. Pacheco Rodriguez, ${ }^{142}$ C. Padilla Aranda, ${ }^{14}$ S. Pagan Griso, ${ }^{18}$ M. Paganini, ${ }^{181}$ G. Palacino, ${ }^{63}$ S. Palazzo, ${ }^{40 b, 40 a}$ S. Palestini, ${ }^{35}$ M. Palka, ${ }^{81 b}$ D. Pallin, ${ }^{37}$ I. Panagoulias, ${ }^{10}$ C. E. Pandini, ${ }^{35}$ J. G. Panduro Vazquez, ${ }^{91}$ P. Pani,${ }^{35}$ L. Paolozzi,${ }^{52}$ T. D. Papadopoulou, ${ }^{10}$ K. Papageorgiou, ${ }^{9, s}$ A. Paramonov, ${ }^{6}$ D. Paredes Hernandez, ${ }^{61 \mathrm{~b}}$ B. Parida, ${ }^{58 \mathrm{c}}$ A. J. Parker, ${ }^{87}$ K. A. Parker, ${ }^{44}$ M. A. Parker, ${ }^{31}$ F. Parodi, ${ }^{53 \mathrm{~b}, 53 \mathrm{a}}$ J. A. Parsons, ${ }^{38}$ U. Parzefall,${ }^{50}$ V. R. Pascuzzi, ${ }^{165}$ J. M. P. Pasner, ${ }^{143}$ E. Pasqualucci, ${ }^{70 a}$ S. Passaggio, ${ }^{53 b}$ F. Pastore, ${ }^{91}$ P. Pasuwan, ${ }^{43 a, 43 b}$ S. Pataraia, ${ }^{97}$ J. R. Pater, ${ }^{98}$ A. Pathak, ${ }^{179, e}$ T. Pauly, ${ }^{35}$ B. Pearson, ${ }^{113}$ M. Pedersen, ${ }^{130}$ S. Pedraza Lopez, ${ }^{172}$ R. Pedro, ${ }^{136 a, 136 b}$ S. V. Peleganchuk, ${ }^{120 b, 120 a}$ O. Penc, ${ }^{137}$ C. Peng, ${ }^{15 d}$ H. Peng, ${ }^{58 a}$ B. S. Peralva, ${ }^{78 a}$ M. M. Perego, ${ }^{142}$ A. P. Pereira Peixoto, ${ }^{136 a}$ D. V. Perepelitsa, ${ }^{29}$ F. Peri, ${ }^{19}$ L. Perini,${ }^{66 a, 66 b}$ H. Pernegger, ${ }^{35}$ S. Perrella,${ }^{67 a, 67 b}$ V. D. Peshekhonov, ${ }^{77, a}$ K. Peters, ${ }^{44}$ R. F. Y. Peters, ${ }^{98}$ B. A. Petersen, ${ }^{35}$ T. C. Petersen, ${ }^{39}$ E. Petit,${ }^{56}$ A. Petridis, ${ }^{1}$ C. Petridou, ${ }^{160}$ P. Petroff, ${ }^{128}$ E. Petrolo, ${ }^{70 a}$ M. Petrov, ${ }^{131}$ F. Petrucci, ${ }^{72 a, 72 b}$ N. E. Pettersson, ${ }^{100}$ A. Peyaud, ${ }^{142}$ R. Pezoa, ${ }^{144 b}$ T. Pham, ${ }^{102}$ F. H. Phillips, ${ }^{104}$ P. W. Phillips, ${ }^{141}$ G. Piacquadio, ${ }^{152}$ E. Pianori, ${ }^{18}$ A. Picazio, ${ }^{100}$ M. A. Pickering, ${ }^{131}$ R. Piegaia, ${ }^{30}$

J. E. Pilcher, ${ }^{36}$ A. D. Pilkington, ${ }^{98}$ M. Pinamonti, ${ }^{71,71 b}$ J. L. Pinfold, ${ }^{3}$ M. Pitt, ${ }^{178}$ M-A. Pleier, ${ }^{29}$ V. Pleskot,${ }^{139}$ E. Plotnikova, ${ }^{77}$ D. Pluth,${ }^{76}$ P. Podberezko, ${ }^{120 b, 120 a}$ R. Poettgen,${ }^{94}$ R. Poggi,${ }^{68 a, 68 b}$ L. Poggioli, ${ }^{128}$ I. Pogrebnyak, ${ }^{104}$ D. Pohl, ${ }^{24}$ I. Pokharel, ${ }^{51}$ G. Polesello, ${ }^{68 a}$ A. Poley, ${ }^{44}$ A. Policicchio, ${ }^{40 b, 40 a}$ R. Polifka, ${ }^{35}$ A. Polini, ${ }^{23 b}$ C. S. Pollard, ${ }^{44}$ V. Polychronakos, ${ }^{29}$ D. Ponomarenko, ${ }^{110}$ L. Pontecorvo ${ }^{70 a}$ G. A. Popeneciu, ${ }^{27 d}$ D. M. Portillo Quintero, ${ }^{132}$ S. Pospisil, ${ }^{138}$ K. Potamianos, ${ }^{44}$ I. N. Potrap,${ }^{77}$ C. J. Potter ${ }^{31}$ H. Potti,${ }^{11}$ T. Poulsen, ${ }^{94}$ J. Poveda,${ }^{35}$ T. D. Powell, ${ }^{146}$ M. E. Pozo Astigarraga, ${ }^{35}$ P. Pralavorio, ${ }^{99}$ S. Prell, ${ }^{76}$ D. Price, ${ }^{98}$ M. Primavera, ${ }^{65 \mathrm{a}}$ S. Prince, ${ }^{101}$ N. Proklova,${ }^{110}$ K. Prokofiev,${ }^{61 \mathrm{c}}$ F. Prokoshin, ${ }^{144 \mathrm{~b}}$ S. Protopopescu, ${ }^{29}$ J. Proudfoot, ${ }^{6}$ M. Przybycien, ${ }^{81 a}$ A. Puri, ${ }^{171}$ P. Puzo, ${ }^{128}$ J. Qian, ${ }^{103}$ Y. Qin, ${ }^{98}$ A. Quadt,${ }^{51}$ M. Queitsch-Maitland, ${ }^{44}$

A. Qureshi, ${ }^{1}$ P. Rados, ${ }^{102}$ F. Ragusa, ${ }^{66,66 \mathrm{~b}}$ G. Rahal, ${ }^{95}$ J. A. Raine, ${ }^{98}$ S. Rajagopalan, ${ }^{29}$ T. Rashid, ${ }^{128}$ S. Raspopov, ${ }^{5}$ M. G. Ratti, ${ }^{66 a 66 b}$ D. M. Rauch, ${ }^{44}$ F. Rauscher, ${ }^{112}$ S. Rave, ${ }^{97}$ B. Ravina, ${ }^{146}$ I. Ravinovich, ${ }^{178}$ J. H. Rawling, ${ }^{98}$ M. Raymond ${ }^{35}$ A. L. Read, ${ }^{130}$ N. P. Readioff,${ }^{56}$ M. Reale, ${ }^{65 a, 65 b}$ D. M. Rebuzzi ${ }^{68 a, 68 b}$ A. Redelbach, ${ }^{175}$ G. Redlinger, ${ }^{29}$ R. Reece, ${ }^{143}$ R. G. Reed ${ }^{32 \mathrm{c}}$ K. Reeves,${ }^{42}$ L. Rehnisch, ${ }^{19}$ J. Reichert, ${ }^{133}$ A. Reiss,${ }^{97}$ C. Rembser, ${ }^{35}$ H. Ren, ${ }^{15 \mathrm{~d}}$ M. Rescigno, ${ }^{70 a}$ S. Resconi ${ }^{66 a}$ E. D. Resseguie, ${ }^{133}$ S. Rettie, ${ }^{173}$ E. Reynolds, ${ }^{21}$ O. L. Rezanova, ${ }^{120 b, 120 a}$ P. Reznicek, ${ }^{139}$ R. Richter, ${ }^{113}$ S. Richter, ${ }^{92}$ E. Richter-Was, ${ }^{81 b}$ O. Ricken, ${ }^{24}$ M. Ridel, ${ }^{132}$ P. Rieck, ${ }^{113}$ C. J. Riegel,${ }^{180}$ O. Rifki, ${ }^{44}$ M. Rijssenbeek,${ }^{152}$ A. Rimoldi, ${ }^{68 a, 68 b}$ M. Rimoldi, ${ }^{20}$ L. Rinaldi, ${ }^{23 b}$ G. Ripellino, ${ }^{151}$ B. Ristić, ${ }^{87}$ E. Ritsch, ${ }^{35}$ I. Riu, ${ }^{14}$ J. C. Rivera Vergara, ${ }^{144 a}$ F. Rizatdinova, ${ }^{125}$ E. Rizvi, ${ }^{90}$ C. Rizzi, ${ }^{14}$ R. T. Roberts, ${ }^{98}$ S. H. Robertson, ${ }^{101, \mathrm{~m}}$ A. Robichaud-Veronneau, ${ }^{101}$ D. Robinson, ${ }^{31}$ J. E. M. Robinson,${ }^{44}$ A. Robson, ${ }^{55}$ E. Rocco, ${ }^{97}$ C. Roda,${ }^{69,69 b}$ Y. Rodina, ${ }^{99}$ S. Rodriguez Bosca, ${ }^{172}$ A. Rodriguez Perez, ${ }^{14}$

D. Rodriguez Rodriguez, ${ }^{172}$ A. M. Rodríguez Vera, ${ }^{166 \mathrm{~b}}$ S. Roe,${ }^{35}$ C. S. Rogan, ${ }^{57}$ O. Røhne, ${ }^{130}$ R. Röhrig, ${ }^{113}$

C. P. A. Roland, ${ }^{63}$ J. Roloff, ${ }^{57}$ A. Romaniouk, ${ }^{110}$ M. Romano, ${ }^{23 b, 23 a}$ N. Rompotis, ${ }^{88}$ M. Ronzani, ${ }^{121}$ L. Roos, ${ }^{132}$ S. Rosati, ${ }^{70 a}$ K. Rosbach, ${ }^{50}$ P. Rose, ${ }^{143}$ N-A. Rosien, ${ }^{51}$ E. Rossi, ${ }^{67 a, 67 b}$ L. P. Rossi,${ }^{53 b}$ L. Rossini, ${ }^{66,66 b}$ J. H. N. Rosten, ${ }^{31}$ R. Rosten, ${ }^{145}$ M. Rotaru, ${ }^{27 b}$ J. Rothberg, ${ }^{145}$ D. Rousseau, ${ }^{128}$ D. Roy ${ }^{32 \mathrm{c}}$ A. Rozanov, ${ }^{99}$ Y. Rozen, ${ }^{158}$ X. Ruan, ${ }^{32 \mathrm{c}}$ F. Rubbo, ${ }^{150}$ F. Rühr, ${ }^{50}$

A. Ruiz-Martinez, ${ }^{33}$ Z. Rurikova, ${ }^{50}$ N. A. Rusakovich, ${ }^{77}$ H. L. Russell, ${ }^{101}$ J. P. Rutherfoord, ${ }^{7}$ N. Ruthmann, ${ }^{35}$

E. M. Rüttinger, ${ }^{44, m m}$ Y. F. Ryabov, ${ }^{134}$ M. Rybar, ${ }^{171}$ G. Rybkin, ${ }^{128}$ S. Ryu,${ }^{6}$ A. Ryzhov,${ }^{140}$ G. F. Rzehorz,${ }^{51}$ P. Sabatini ${ }^{51}$ G. Sabato, ${ }^{118}$ S. Sacerdoti, ${ }^{128}$ H. F-W. Sadrozinski, ${ }^{143}$ R. Sadykov, ${ }^{77}$ F. Safai Tehrani, ${ }^{70 a}$ P. Saha, ${ }^{119}$ M. Sahinsoy, ${ }^{59 a}$ A. Sahu, ${ }^{180}$ M. Saimpert, ${ }^{44}$ M. Saito, ${ }^{161}$ T. Saito, ${ }^{161}$ H. Sakamoto, ${ }^{161}$ A. Sakharov, ${ }^{121, e e}$ D. Salamani, ${ }^{52}$ G. Salamanna, ${ }^{72 a, 72 b}$ J. E. Salazar Loyola, ${ }^{14 b \mathrm{~b}}$ D. Salek, ${ }^{118}$ P. H. Sales De Bruin, ${ }^{170}$ D. Salihagic, ${ }^{113}$ A. Salnikov, ${ }^{150}$ J. Salt, ${ }^{172}$ D. Salvatore, ${ }^{40 b, 40 a}$ F. Salvatore, ${ }^{153}$ A. Salvucci, ${ }^{61 \mathrm{a}, 61 \mathrm{~b}, 61 \mathrm{c}}$ A. Salzburger, ${ }^{35}$ D. Sammel,${ }^{50}$ D. Sampsonidis, ${ }^{160}$ D. Sampsonidou, ${ }^{160}$ J. Sánchez, ${ }^{172}$ A. Sanchez Pineda, ${ }^{64 a, 64 c}$ H. Sandaker, ${ }^{130}$ C. O. Sander, ${ }^{44}$ M. Sandhoff, ${ }^{180}$ C. Sandoval, ${ }^{22}$ D. P. C. Sankey, ${ }^{141}$ M. Sannino, ${ }^{53 b, 53 a}$ Y. Sano, ${ }^{115}$ A. Sansoni,${ }^{49}$ C. Santoni, ${ }^{37}$ H. Santos, ${ }^{136 a}$ I. Santoyo Castillo, ${ }^{153}$ A. Sapronov, ${ }^{77}$ J. G. Saraiva, ${ }^{136 a, 136 d}$ O. Sasaki, ${ }^{79}$ K. Sato, ${ }^{167}$ E. Sauvan, ${ }^{5}$ P. Savard,${ }^{165, d}$ N. Savic, ${ }^{113}$ R. Sawada, ${ }^{161}$ C. Sawyer, ${ }^{141}$ L. Sawyer, ${ }^{93, u}$ C. Sbarra, ${ }^{23 b}$ A. Sbrizzi, ${ }^{23 b, 23 a}$ T. Scanlon, ${ }^{92}$ D. A. Scannicchio, ${ }^{169}$ J. Schaarschmidt, ${ }^{145}$ P. Schacht, ${ }^{113}$ B. M. Schachtner, ${ }^{112}$ D. Schaefer, ${ }^{36}$ L. Schaefer, ${ }^{133}$ J. Schaeffer, ${ }^{97}$ S. Schaepe, ${ }^{35}$ U. Schäfer, ${ }^{97}$ A. C. Schaffer, ${ }^{128}$ 
D. Schaile, ${ }^{112}$ R. D. Schamberger, ${ }^{152}$ N. Scharmberg, ${ }^{98}$ V. A. Schegelsky, ${ }^{134}$ D. Scheirich, ${ }^{139}$ F. Schenck, ${ }^{19}$ M. Schernau, ${ }^{169}$ C. Schiavi, ${ }^{53 b, 53 a}$ S. Schier, ${ }^{143}$ L. K. Schildgen ${ }^{24}$ Z. M. Schillaci ${ }^{26}$ E. J. Schioppa,${ }^{35}$ M. Schioppa, ${ }^{40 b, 40 a}$ K. E. Schleicher ${ }^{50}$ S. Schlenker, ${ }^{35}$ K. R. Schmidt-Sommerfeld, ${ }^{113}$ K. Schmieden, ${ }^{35}$ C. Schmitt, ${ }^{97}$ S. Schmitt, ${ }^{44}$ S. Schmitz, ${ }^{97}$ U. Schnoor,${ }^{50}$ L. Schoeffel, ${ }^{142}$ A. Schoening, ${ }^{59 b}$ E. Schopf,${ }^{24}$ M. Schott, ${ }^{97}$ J. F. P. Schouwenberg, ${ }^{117}$ J. Schovancova, ${ }^{35}$ S. Schramm, ${ }^{52}$ N. Schuh, ${ }^{97}$ A. Schulte, ${ }^{97}$ H-C. Schultz-Coulon, ${ }^{59 a}$ M. Schumacher, ${ }^{50}$ B. A. Schumm, ${ }^{143}$ Ph. Schune, ${ }^{142}$ A. Schwartzman, ${ }^{150}$ T. A. Schwarz, ${ }^{103}$ H. Schweiger,${ }^{98}$ Ph. Schwemling, ${ }^{142}$ R. Schwienhorst, ${ }^{104}$ A. Sciandra, ${ }^{24}$ G. Sciolla, ${ }^{26}$ M. Scornajenghi, ${ }^{40 b, 40 a}$ F. Scuri ${ }^{69 a}$ F. Scutti, ${ }^{102}$ L. M. Scyboz,${ }^{113}$ J. Searcy,${ }^{103}$ C. D. Sebastiani, ${ }^{70 a, 70 b}$ P. Seema, ${ }^{24}$ S. C. Seidel, ${ }^{116}$ A. Seiden, ${ }^{143}$ T. Seiss,${ }^{36}$ J. M. Seixas, ${ }^{78 b}$ G. Sekhniaidze, ${ }^{67 a}$ K. Sekhon, ${ }^{103}$ S. J. Sekula, ${ }^{41}$ N. Semprini-Cesari, ${ }^{23 b, 23 a}$ S. Sen, ${ }^{47}$ S. Senkin, ${ }^{37}$ C. Serfon, ${ }^{130}$ L. Serin, ${ }^{128}$ L. Serkin, ${ }^{64 a 64 b}$ M. Sessa, ${ }^{72 a, 72 b}$ H. Severini, ${ }^{124}$ F. Sforza, ${ }^{168}$ A. Sfyrla, ${ }^{52}$ E. Shabalina, ${ }^{51}$ J. D. Shahinian, ${ }^{143}$ N. W. Shaikh, ${ }^{43 a, 43 b}$ L. Y. Shan, ${ }^{15 a}$ R. Shang, ${ }^{171}$ J. T. Shank, ${ }^{25}$ M. Shapiro, ${ }^{18}$ A. S. Sharma, ${ }^{1}$ A. Sharma, ${ }^{131}$ P. B. Shatalov, ${ }^{109}$ K. Shaw,${ }^{64 a 64 b}$ S. M. Shaw, ${ }^{98}$ A. Shcherbakova, ${ }^{134}$ C. Y. Shehu, ${ }^{153}$ Y. Shen, ${ }^{124}$ N. Sherafati, ${ }^{33}$ A. D. Sherman, ${ }^{25}$ P. Sherwood, ${ }^{92}$ L. Shi, ${ }^{155, \text { nn }}$ S. Shimizu, ${ }^{80}$ C. O. Shimmin, ${ }^{181}$ M. Shimojima, ${ }^{114}$ I. P. J. Shipsey, ${ }^{131}$ S. Shirabe, ${ }^{85}$ M. Shiyakova, ${ }^{77}$ J. Shlomi, ${ }^{178}$ A. Shmeleva, ${ }^{108}$ D. Shoaleh Saadi, ${ }^{107}$ M. J. Shochet, ${ }^{36}$ S. Shojaii, ${ }^{102}$ D. R. Shope, ${ }^{124}$ S. Shrestha, ${ }^{122}$ E. Shulga, ${ }^{110}$ P. Sicho, ${ }^{137}$ A. M. Sickles, ${ }^{171}$ P. E. Sidebo, ${ }^{151}$ E. Sideras Haddad, ${ }^{32 c}$ O. Sidiropoulou, ${ }^{175}$ A. Sidoti, ${ }^{23 b, 23 a}$ F. Siegert, ${ }^{46}$ Dj. Sijacki, ${ }^{16}$ J. Silva, ${ }^{136 a}$ M. Silva Jr., ${ }^{179}$

S. B. Silverstein, ${ }^{43 a}$ L. Simic, ${ }^{77}$ S. Simion, ${ }^{128}$ E. Simioni,${ }^{97}$ M. Simon,${ }^{97}$ P. Sinervo, ${ }^{165}$ N. B. Sinev, ${ }^{127}$ M. Sioli, ${ }^{23 b, 23 a}$ G. Siragusa, ${ }^{175}$ I. Siral, ${ }^{103}$ S.Yu. Sivoklokov, ${ }^{111}$ J. Sjölin, ${ }^{43 a, 43 b}$ M. B. Skinner, ${ }^{87}$ P. Skubic, ${ }^{124}$ M. Slater, ${ }^{21}$ T. Slavicek, ${ }^{138}$ M. Slawinska, ${ }^{82}$ K. Sliwa, ${ }^{168}$ R. Slovak, ${ }^{139}$ V. Smakhtin, ${ }^{178}$ B. H. Smart, ${ }^{5}$ J. Smiesko, ${ }^{28 a}$ N. Smirnov, ${ }^{110}$ S.Yu. Smirnov, ${ }^{110}$ Y. Smirnov, ${ }^{110}$ L. N. Smirnova, ${ }^{111}$ O. Smirnova, ${ }^{94}$ J. W. Smith, ${ }^{51}$ M. N. K. Smith,${ }^{38}$ R. W. Smith, ${ }^{38}$ M. Smizanska, ${ }^{87}$ K. Smolek, ${ }^{138}$ A. A. Snesarev ${ }^{108}$ I. M. Snyder ${ }^{127}$ S. Snyder, ${ }^{29}$ R. Sobie, ${ }^{174, m}$ A. M. Soffa, ${ }^{169}$ A. Soffer ${ }^{159}$ A. Søgaard, ${ }^{48}$ D. A. Soh, ${ }^{155}$ G. Sokhrannyi, ${ }^{89}$ C. A. Solans Sanchez, ${ }^{35}$ M. Solar, ${ }^{138}$ E. Yu. Soldatov, ${ }^{110}$ U. Soldevila, ${ }^{172}$ A. A. Solodkov, ${ }^{140}$ A. Soloshenko, ${ }^{77}$ O. V. Solovyanov,${ }^{140}$ V. Solovyev, ${ }^{134}$ P. Sommer, ${ }^{146}$ H. Son, ${ }^{168}$ W. Song, ${ }^{141}$ A. Sopczak, ${ }^{138}$ F. Sopkova ${ }^{28 b}$

D. Sosa, ${ }^{59 b}$ C. L. Sotiropoulou, ${ }^{69 a, 69 b}$ S. Sottocornola,${ }^{68 a, 68 b}$ R. Soualah, ${ }^{64 a, 64 c, 00}$ A. M. Soukharev, ${ }^{120 b, 120 a}$ D. South, ${ }^{44}$ B. C. Sowden, ${ }^{91}$ S. Spagnolo, ${ }^{65 a, 65 b}$ M. Spalla, ${ }^{113}$ M. Spangenberg, ${ }^{176}$ F. Spanò, ${ }^{91}$ D. Sperlich, ${ }^{19}$ F. Spettel, ${ }^{113}$ T. M. Spieker, ${ }^{59 a}$ R. Spighi, ${ }^{23 b}$ G. Spigo, ${ }^{35}$ L. A. Spiller, ${ }^{102}$ M. Spousta, ${ }^{139}$ A. Stabile, ${ }^{66 a, 66 b}$ R. Stamen, ${ }^{59 a}$ S. Stamm, ${ }^{19}$ E. Stanecka, ${ }^{82}$ R. W. Stanek, ${ }^{6}$ C. Stanescu, ${ }^{72 a}$ M. M. Stanitzki, ${ }^{44}$ B. S. Stapf,${ }^{118}$ S. Stapnes, ${ }^{130}$ E. A. Starchenko, ${ }^{140}$

G. H. Stark ${ }^{36}$ J. Stark, ${ }^{56}$ S. H. Stark,${ }^{39}$ P. Staroba, ${ }^{137}$ P. Starovoitov, ${ }^{59 a}$ S. Stärz,${ }^{35}$ R. Staszewski, ${ }^{82}$ M. Stegler, ${ }^{44}$ P. Steinberg, ${ }^{29}$ B. Stelzer, ${ }^{149}$ H. J. Stelzer, ${ }^{35}$ O. Stelzer-Chilton, ${ }^{166 a}$ H. Stenzel,${ }^{54}$ T. J. Stevenson, ${ }^{90}$ G. A. Stewart ${ }^{55}$ M. C. Stockton, ${ }^{127}$ G. Stoicea, ${ }^{27 b}$ P. Stolte, ${ }^{51}$ S. Stonjek, ${ }^{113}$ A. Straessner, ${ }^{46}$ J. Strandberg, ${ }^{151}$ S. Strandberg, ${ }^{43 a, 43 b}$ M. Strauss, ${ }^{124}$ P. Strizenec, ${ }^{28 b}$ R. Ströhmer, ${ }^{175}$ D. M. Strom, ${ }^{127}$ R. Stroynowski, ${ }^{41}$ A. Strubig, ${ }^{48}$ S. A. Stucci, ${ }^{29}$ B. Stugu, ${ }^{17}$ J. Stupak, ${ }^{124}$ N. A. Styles, ${ }^{44}$ D. Su, ${ }^{150}$ J. Su, ${ }^{135}$ S. Suchek ${ }^{59 a}$ Y. Sugaya,${ }^{129}$ M. Suk, ${ }^{138}$ V. V. Sulin, ${ }^{108}$ D. M. S. Sultan, ${ }^{52}$ S. Sultansoy, ${ }^{4 c}$ T. Sumida, ${ }^{83}$ S. Sun, ${ }^{103}$ X. Sun, ${ }^{3}$ K. Suruliz, ${ }^{153}$ C. J. E. Suster, ${ }^{154}$ M. R. Sutton, ${ }^{153}$ S. Suzuki, ${ }^{79}$ M. Svatos, ${ }^{137}$ M. Swiatlowski, ${ }^{36}$ S. P. Swift, ${ }^{2}$ A. Sydorenko, ${ }^{97}$ I. Sykora, ${ }^{28 a}$ T. Sykora, ${ }^{139}$ D. Ta, ${ }^{97}$ K. Tackmann,,${ }^{44, p p}$ J. Taenzer, ${ }^{159}$ A. Taffard, ${ }^{169}$ R. Tafirout, ${ }^{166 a}$ E. Tahirovic, ${ }^{90}$ N. Taiblum, ${ }^{159}$ H. Takai, ${ }^{29}$ R. Takashima, ${ }^{84}$ E. H. Takasugi,${ }^{113}$ K. Takeda, ${ }^{80}$ T. Takeshita, ${ }^{147}$ Y. Takubo,${ }^{79}$ M. Talby, ${ }^{99}$ A. A. Talyshev, ${ }^{120 b, 120 a}$ J. Tanaka, ${ }^{161}$ M. Tanaka, ${ }^{163}$ R. Tanaka, ${ }^{128}$ R. Tanioka ${ }^{80}$ B. B. Tannenwald, ${ }^{122}$ S. Tapia Araya, ${ }^{144 \mathrm{~b}}$ S. Tapprogge, ${ }^{97}$ A. Tarek Abouelfadl Mohamed, ${ }^{132}$ S. Tarem, ${ }^{158}$ G. Tarna, ${ }^{27 b, p}$ G. F. Tartarelli, ${ }^{66 \mathrm{a}}$ P. Tas, ${ }^{139}$ M. Tasevsky, ${ }^{137}$ T. Tashiro, ${ }^{83}$ E. Tassi,${ }^{40 b, 40 a}$ A. Tavares Delgado, ${ }^{136 a, 136 b}$ Y. Tayalati, ${ }^{34 \mathrm{e}}$ A. C. Taylor, ${ }^{116}$ A. J. Taylor, ${ }^{48}$ G. N. Taylor, ${ }^{102}$ P. T. E. Taylor, ${ }^{102}$ W. Taylor,${ }^{166 b}$ A. S. Tee,${ }^{87}$ P. Teixeira-Dias,${ }^{91}$ D. Temple,${ }^{149}$ H. Ten Kate, ${ }^{35}$ P. K. Teng, ${ }^{155}$ J. J. Teoh, ${ }^{129}$ F. Tepel, ${ }^{180}$ S. Terada,${ }^{79}$ K. Terashi, ${ }^{161}$ J. Terron, ${ }^{96}$ S. Terzo, ${ }^{14}$ M. Testa, ${ }^{49}$ R. J. Teuscher, ${ }^{165, \mathrm{~m}}$ S. J. Thais, ${ }^{181}$ T. Theveneaux-Pelzer, ${ }^{44}$ F. Thiele, ${ }^{39}$ J. P. Thomas, ${ }^{21}$ A. S. Thompson, ${ }^{55}$ P. D. Thompson, ${ }^{21}$ L. A. Thomsen, ${ }^{181}$ E. Thomson, ${ }^{133}$ Y. Tian, ${ }^{38}$ R. E. Ticse Torres, ${ }^{51}$ V. O. Tikhomirov, ${ }^{108, q q}$ Yu.A. Tikhonov, ${ }^{120 b, 120 a}$ S. Timoshenko, ${ }_{110}$ P. Tipton, ${ }^{181}$ S. Tisserant, ${ }^{99}$ K. Todome, ${ }^{163}$ S. Todorova-Nova, ${ }^{5}$ S. Todt, ${ }^{46}$ J. Tojo,${ }^{85}$ S. Tokár ${ }^{28 a}$ K. Tokushuku, ${ }^{79}$ E. Tolley, ${ }^{122}$ M. Tomoto, ${ }^{115}$ L. Tompkins, ${ }^{150} \mathrm{~K}$. Toms, ${ }^{116}$ B. Tong, ${ }^{57}$ P. Tornambe ${ }^{50}$ E. Torrence, ${ }^{127}$ H. Torres ${ }^{46}$ E. Torró Pastor, ${ }^{145}$ C. Tosciri, ${ }^{131}$ J. Toth, ${ }^{99, r r}$ F. Touchard, ${ }^{99}$ D. R. Tovey, ${ }^{146}$ C. J. Treado, ${ }^{121}$ T. Trefzger, ${ }^{175}$ F. Tresoldi, ${ }^{153}$ A. Tricoli, ${ }^{29}$ I. M. Trigger, ${ }^{166 a}$ S. Trincaz-Duvoid, ${ }^{132}$ M. F. Tripiana,${ }^{14}$ W. Trischuk, ${ }^{165}$ B. Trocmé, ${ }^{56}$ A. Trofymov, ${ }^{128}$ C. Troncon, ${ }^{66 a}$ M. Trovatelli, ${ }^{174}$ F. Trovato, ${ }^{153}$ L. Truong, ${ }^{32 b}$ M. Trzebinski, ${ }^{82}$ A. Trzupek, ${ }^{82}$ F. Tsai, ${ }^{44}$ J.C-L. Tseng, ${ }^{131}$ P. V. Tsiareshka, ${ }^{105}$ N. Tsirintanis, ${ }^{9}$ V. Tsiskaridze, ${ }^{152}$ E. G. Tskhadadze, ${ }^{157 a}$ I. I. Tsukerman,${ }^{109}$ V. Tsulaia, ${ }^{18}$ S. Tsuno, ${ }^{79}$ D. Tsybychev, ${ }^{152}$ Y. Tu, ${ }^{61 b}$ A. Tudorache, ${ }^{27 b}$ V. Tudorache, ${ }^{27 b}$ T. T. Tulbure, ${ }^{27 \mathrm{a}}$ A. N. Tuna, ${ }^{57}$ S. Turchikhin, ${ }^{77}$ D. Turgeman, ${ }^{178}$ I. Turk Cakir ${ }^{4 b, s s}$ R. Turra, ${ }^{66 a}$ P. M. Tuts,${ }^{38}$ E. Tzovara, ${ }^{97}$ G. Ucchielli, ${ }^{23 b, 23 a}$ I. Ueda, ${ }^{79}$ 
M. Ughetto, ${ }^{43 a, 43 b}$ F. Ukegawa, ${ }^{167}$ G. Unal, ${ }^{35}$ A. Undrus, ${ }^{29}$ G. Unel, ${ }^{169}$ F. C. Ungaro, ${ }^{102}$ Y. Unno, ${ }^{79}$ K. Uno, ${ }^{161}$ J. Urban, ${ }^{28 b}$ P. Urquijo, ${ }^{102}$ P. Urrejola, ${ }^{97}$ G. Usai, ${ }^{8}$ J. Usui, ${ }^{79}$ L. Vacavant, ${ }^{99}$ V. Vacek, ${ }^{138}$ B. Vachon, ${ }^{101}$ K. O. H. Vadla, ${ }^{130}$ A. Vaidya, ${ }^{92}$ C. Valderanis, ${ }^{112}$ E. Valdes Santurio, ${ }^{43 a, 43 b}$ M. Valente, ${ }^{52}$ S. Valentinetti, ${ }^{23 b, 23 a}$ A. Valero, ${ }^{172}$ L. Valéry, ${ }^{44}$ R. A. Vallance,${ }^{21}$ A. Vallier, ${ }^{5}$ J. A. Valls Ferrer, ${ }^{172}$ T. R. Van Daalen, ${ }^{14}$ W. Van Den Wollenberg, ${ }^{118}$ H. Van der Graaf, ${ }^{118}$ P. Van Gemmeren, ${ }^{6}$ J. Van Nieuwkoop, ${ }^{149}$ I. Van Vulpen, ${ }^{118}$ M. C. van Woerden, ${ }^{118}$ M. Vanadia, ${ }^{71 a, 71 b}$ W. Vandelli, ${ }^{35}$ A. Vaniachine, ${ }^{164}$ P. Vankov, ${ }^{118}$ R. Vari, ${ }^{70 a}$ E. W. Varnes, ${ }^{7}$ C. Varni,${ }^{53 b, 53 a}$ T. Varol,${ }^{41}$ D. Varouchas,${ }^{128}$ A. Vartapetian, ${ }^{8}$ K. E. Varvell, ${ }^{154}$ G. A. Vasquez ${ }^{144 \mathrm{~b}}$ J. G. Vasquez, ${ }^{181}$ F. Vazeille ${ }^{37}$ D. Vazquez Furelos, ${ }^{14}$ T. Vazquez Schroeder, ${ }^{101}$ J. Veatch, ${ }^{51}$ V. Vecchio, ${ }^{72 a, 72 b}$ L. M. Veloce, ${ }^{165}$ F. Veloso, ${ }^{136 a, 136 c}$ S. Veneziano, ${ }^{70 a}$ A. Ventura,${ }^{65 a, 65 b}$ M. Venturi,,${ }^{174}$ N. Venturi, ${ }^{35}$ V. Vercesi, ${ }^{68 \mathrm{a}}$ M. Verducci, ${ }^{72 \mathrm{a}, 72 \mathrm{~b}}$ W. Verkerke, ${ }^{118}$ A. T. Vermeulen, ${ }^{118}$ J. C. Vermeulen, ${ }^{118}$ M. C. Vetterli, ${ }^{149, d}$

N. Viaux Maira, ${ }^{144 \mathrm{~b}}$ O. Viazlo, ${ }^{94}$ I. Vichou, ${ }^{171, \mathrm{a}}$ T. Vickey, ${ }^{146}$ O. E. Vickey Boeriu, ${ }^{146}$ G. H. A. Viehhauser, ${ }^{131}$ S. Viel, ${ }^{18}$ L. Vigani, ${ }^{131}$ M. Villa, ${ }^{23 b, 23 a}$ M. Villaplana Perez,${ }^{66,66 b}$ E. Vilucchi, ${ }^{49}$ M. G. Vincter, ${ }^{33}$ V. B. Vinogradov,${ }^{77}$ A. Vishwakarma ${ }^{44}$ C. Vittori, ${ }^{23 b, 23 a}$ I. Vivarelli, ${ }^{153} \mathrm{~S}$. Vlachos,${ }^{10} \mathrm{M}$. Vogel, ${ }^{180} \mathrm{P}$. Vokac, ${ }^{138} \mathrm{G}$. Volpi,${ }^{14}$ S. E. Von Buddenbrock, ${ }^{32 c}$ E. Von Toerne, ${ }^{24}$ V. Vorobel,,${ }^{139}$ K. Vorobev,${ }^{110}$ M. Vos,${ }^{172}$ J. H. Vossebeld,${ }^{88}$ N. Vranjes, ${ }^{16}$ M. Vranjes Milosavljevic, ${ }^{16}$ V. Vrba ${ }^{138}$ M. Vreeswijk, ${ }^{118}$ T. Šfiligoj, ${ }^{89}$ R. Vuillermet, ${ }^{35}$ I. Vukotic, ${ }^{36}$ T. Ženišs, ${ }^{28 a}$ L. Živković, ${ }^{16}$ P. Wagner, ${ }^{24}$ W. Wagner, ${ }^{180}$ J. Wagner-Kuhr, ${ }^{112} \mathrm{H}$. Wahlberg, ${ }^{86} \mathrm{~S}$. Wahrmund, ${ }^{46} \mathrm{~K}$. Wakamiya,${ }^{80} \mathrm{~J}$. Walder ${ }^{87}$ R. Walker, ${ }^{112}$ W. Walkowiak, ${ }^{148}$ V. Wallangen, ${ }^{43 a, 43 b}$ A. M. Wang, ${ }^{57}$ C. Wang, ${ }^{58 b, p}$ F. Wang, ${ }^{179}$ H. Wang, ${ }^{18}$ H. Wang, ${ }^{3}$ J. Wang, ${ }^{154}$ J. Wang, ${ }^{59 b}$ P. Wang, ${ }^{41}$ Q. Wang, ${ }^{124}$ R.-J. Wang, ${ }^{132}$ R. Wang, ${ }^{58 \mathrm{a}}$ R. Wang, ${ }^{6}$ S. M. Wang, ${ }^{155}$ W. Wang, ${ }^{155, t t}$ W. X. Wang, ${ }^{58 a, u u}$ Y. Wang, ${ }^{58 a}$ Z. Wang, ${ }^{58 c}$ C. Wanotayaroj, ${ }^{44}$ A. Warburton, ${ }^{101}$ C. P. Ward ${ }^{31}$ D. R. Wardrope,${ }^{92}$ A. Washbrook, ${ }^{48}$ P. M. Watkins, ${ }^{21}$ A. T. Watson, ${ }^{21}$ M. F. Watson, ${ }^{21}$ G. Watts, ${ }^{145}$ S. Watts ${ }^{98}$ B. M. Waugh ${ }^{92}$ A. F. Webb, ${ }^{11}$ S. Webb, ${ }^{97}$ C. Weber, ${ }^{181}$ M. S. Weber, ${ }^{20}$ S. A. Weber, ${ }^{33}$ S. M. Weber, ${ }^{59 a}$ J. S. Webster, ${ }^{6}$ A. R. Weidberg, ${ }^{131}$ B. Weinert, ${ }^{63}$ J. Weingarten, ${ }^{51}$ M. Weirich, ${ }^{97}$ C. Weiser, ${ }^{50}$ P. S. Wells, ${ }^{35}$ T. Wenaus, ${ }^{29}$ T. Wengler, ${ }^{35}$ S. Wenig,${ }^{35}$ N. Wermes,${ }^{24}$ M. D. Werner, ${ }^{76}$ P. Werner, ${ }^{35}$ M. Wessels, ${ }^{59 a}$ T. D. Weston, ${ }^{20}$ K. Whalen, ${ }^{127}$ N. L. Whallon, ${ }^{145}$ A. M. Wharton, ${ }^{87}$

A. S. White, ${ }^{103}$ A. White, ${ }^{8}$ M. J. White, ${ }^{1}$ R. White ${ }^{144 b}$ D. Whiteson, ${ }^{169}$ B. W. Whitmore,${ }^{87}$ F. J. Wickens,${ }^{141}$ W. Wiedenmann, ${ }^{179}$ M. Wielers, ${ }^{141}$ C. Wiglesworth ${ }^{39}$ L. A. M. Wiik-Fuchs,${ }^{50}$ A. Wildauer, ${ }^{113}$ F. Wilk, ${ }^{98}$ H. G. Wilkens, ${ }^{35}$ H. H. Williams, ${ }^{133}$ S. Williams, ${ }^{31}$ C. Willis, ${ }^{104}$ S. Willocq, ${ }^{100}$ J. A. Wilson, ${ }^{21}$ I. Wingerter-Seez, ${ }^{5}$ E. Winkels, ${ }^{153}$ F. Winklmeier, ${ }^{127}$ O. J. Winston, ${ }^{153}$ B. T. Winter, ${ }^{24}$ M. Wittgen, ${ }^{150}$ M. Wobisch, ${ }^{93}$ A. Wolf,${ }^{97}$ T. M. H. Wolf, ${ }^{118}$ R. Wolff, ${ }^{99}$ M. W. Wolter, ${ }^{82}$ H. Wolters, ${ }^{136 a, 136 c}$ V. W. S. Wong, ${ }^{173}$ N. L. Woods, ${ }^{143}$ S. D. Worm, ${ }^{21}$ B. K. Wosiek,${ }^{82}$ K. W. Woźniak, ${ }^{82}$ K. Wraight, ${ }^{55}$ M. Wu, ${ }^{36}$ S. L. Wu, ${ }^{179}$ X. Wu, ${ }^{52}$ Y. Wu, ${ }^{58 a}$ T. R. Wyatt, ${ }^{98}$ B. M. Wynne, ${ }^{48}$ S. Xella, ${ }^{39}$ Z. Xi, ${ }^{103}$ L. Xia, ${ }^{176}$ D. Xu, ${ }^{15 a}$ H. Xu ${ }^{58 a}$ L. Xu, ${ }^{29}$ T. Xu,${ }^{142}$ W. Xu, ${ }^{103}$ B. Yabsley, ${ }^{154}$ S. Yacoob,${ }^{32 a}$ K. Yajima, ${ }^{129}$ D. P. Yallup,,${ }^{92}$ D. Yamaguchi, ${ }^{163}$ Y. Yamaguchi, ${ }^{163}$ A. Yamamoto, ${ }^{79}$ T. Yamanaka, ${ }^{161}$ F. Yamane,${ }^{80}$ M. Yamatani, ${ }^{161}$ T. Yamazaki, ${ }^{161}$ Y. Yamazaki, ${ }^{80}$ Z. Yan,,${ }^{25}$ H. J. Yang, ${ }^{58 c, 58 d}$ H. T. Yang, ${ }^{18}$ S. Yang, ${ }^{75}$ Y. Yang, ${ }^{161}$ Y. Yang, ${ }^{155}$ Z. Yang, ${ }^{17}$ W-M. Yao, ${ }^{18}$ Y. C. Yap, ${ }^{44}$ Y. Yasu, ${ }^{79}$ E. Yatsenko, ${ }^{5}$ J. Ye ${ }^{41}$ S. Ye ${ }^{29}$ I. Yeletskikh, ${ }^{77}$ E. Yigitbasi, ${ }^{25}$ E. Yildirim, ${ }^{97}$ K. Yorita, ${ }^{177}$ K. Yoshihara ${ }^{133}$ C. J. S. Young, ${ }^{35}$ C. Young, ${ }^{150}$ J. Yu, ${ }^{8}$ J. Yu, ${ }^{76}$ X. Yue, ${ }^{59}$ S. P. Y. Yuen, ${ }^{24}$ I. Yusuff, ${ }^{31, v v}$ B. Zabinski, ${ }^{82}$ G. Zacharis, ${ }^{10}$ E. Zaffaroni, ${ }^{52}$ R. Zaidan, ${ }^{14}$ A. M. Zaitsev, ${ }^{140, j j}$ N. Zakharchuk, ${ }^{44}$ J. Zalieckas, ${ }^{17}$ S. Zambito, ${ }^{57}$ D. Zanzi,${ }^{35}$ D. R. Zaripovas,${ }^{55}$ C. Zeitnitz, ${ }^{180}$ G. Zemaityte, ${ }^{131}$ J. C. Zeng, ${ }^{171}$ Q. Zeng, ${ }^{150}$ O. Zenin, ${ }^{140}$ D. Zerwas, ${ }^{128}$ M. Zgubič,${ }^{131}$ D. F. Zhang, ${ }^{58 b}$ D. Zhang, ${ }^{103}$ F. Zhang, ${ }_{179}$ G. Zhang, ${ }^{58, \text { uu }}$ H. Zhang, ${ }^{15 \mathrm{c}}$ J. Zhang, ${ }^{6}$ L. Zhang, ${ }^{50}$ L. Zhang ${ }^{58 \mathrm{a}}$ M. Zhang, ${ }_{171}$ P. Zhang, ${ }^{15 \mathrm{c}}$ R. Zhang, ${ }^{58 \mathrm{a}, \mathrm{p}}$ R. Zhang, ${ }^{24}$ X. Zhang, ${ }^{58 \mathrm{~b}}$ Y. Zhang, ${ }^{15 \mathrm{~d}}$ Z. Zhang, ${ }^{128}$ X. Zhao, ${ }^{41}$ Y. Zhao, ${ }^{58 b, 128, \mathrm{x}}$ Z. Zhao, ${ }^{58 \mathrm{a}}$ A. Zhemchugov, ${ }^{77}$ B. Zhou, ${ }^{103}$ C. Zhou, ${ }^{179}$ L. Zhou, ${ }^{41}$ M. S. Zhou, ${ }^{15 \mathrm{~d}}$ M. Zhou, ${ }^{152}$ N. Zhou, ${ }^{58 \mathrm{c}}$ Y. Zhou, ${ }^{7}$ C. G. Zhu ${ }^{58 \mathrm{~b}}$ H. L. Zhu, ${ }^{58 \mathrm{a}}$ H. Zhu, ${ }^{15 \mathrm{a}}$ J. Zhu, ${ }^{103}$ Y. Zhu, ${ }^{58 a}$ X. Zhuang, ${ }^{15 a}$ K. Zhukov, ${ }^{108}$ V. Zhulanov, ${ }^{120 b, 120 a}$ A. Zibell, ${ }^{175}$ D. Zieminska, ${ }^{63}$ N. I. Zimine, ${ }^{77}$ S. Zimmermann, ${ }^{50}$ Z. Zinonos, ${ }^{113}$ M. Zinser, ${ }^{97}$ M. Ziolkowski, ${ }^{148}$ G. Zobernig, ${ }^{179}$ A. Zoccoli, ${ }^{23 b, 23 a}$ K. Zoch, ${ }^{51}$ T. G. Zorbas, ${ }^{146}$ R. Zou, ${ }^{36}$ M. Zur Nedden, ${ }^{19}$ and L. Zwalinski ${ }^{35}$

(ATLAS Collaboration)

\footnotetext{
${ }^{1}$ Department of Physics, University of Adelaide, Adelaide, Australia

${ }^{2}$ Physics Department, SUNY Albany, Albany, New York, USA

${ }^{3}$ Department of Physics, University of Alberta, Edmonton Alberta, Canada

${ }^{4 a}$ Department of Physics, Ankara University, Ankara, Turkey

${ }^{4 b}$ Istanbul Aydin University, Istanbul, Turkey
} 
${ }^{4 \mathrm{c}}$ Division of Physics, TOBB University of Economics and Technology, Ankara, Turkey

${ }^{5}$ LAPP, Université Grenoble Alpes, Université Savoie Mont Blanc, CNRS/IN2P3, Annecy, France

${ }^{6}$ High Energy Physics Division, Argonne National Laboratory, Argonne, Illinois, USA

${ }^{7}$ Department of Physics, University of Arizona, Tucson, Arizona, USA

${ }^{8}$ Department of Physics, University of Texas at Arlington, Arlington, Texas, USA

${ }^{9}$ Physics Department, National and Kapodistrian University of Athens, Athens, Greece

${ }^{10}$ Physics Department, National Technical University of Athens, Zografou, Greece

${ }^{11}$ Department of Physics, University of Texas at Austin, Austin, Texas, USA

${ }^{12 a}$ Bahcesehir University, Faculty of Engineering and Natural Sciences, Istanbul, Turkey

${ }^{12 \mathrm{~b}}$ Istanbul Bilgi University, Faculty of Engineering and Natural Sciences, Istanbul, Turkey

${ }^{12 \mathrm{c}}$ Department of Physics, Bogazici University, Istanbul, Turkey

${ }^{12 \mathrm{~d}}$ Department of Physics Engineering, Gaziantep University, Gaziantep, Turkey

${ }^{13}$ Institute of Physics, Azerbaijan Academy of Sciences, Baku, Azerbaijan

${ }^{14}$ Institut de Física d'Altes Energies (IFAE), Barcelona Institute of Science and Technology, Barcelona, Spain

${ }^{15 a}$ Institute of High Energy Physics, Chinese Academy of Sciences, Beijing, China

${ }^{15 \mathrm{~b}}$ Physics Department, Tsinghua University, Beijing, China

${ }^{15 \mathrm{c}}$ Department of Physics, Nanjing University, Nanjing, China

${ }^{15 \mathrm{~d}}$ University of Chinese Academy of Science (UCAS), Beijing, China

${ }^{16}$ Institute of Physics, University of Belgrade, Belgrade, Serbia

${ }^{17}$ Department for Physics and Technology, University of Bergen, Bergen, Norway

${ }^{18}$ Physics Division, Lawrence Berkeley National Laboratory and University of California, Berkeley, California, USA

${ }^{19}$ Institut für Physik, Humboldt Universität zu Berlin, Berlin, Germany

${ }^{20}$ Albert Einstein Center for Fundamental Physics and Laboratory for High Energy Physics, University of Bern, Bern, Switzerland

${ }^{21}$ School of Physics and Astronomy, University of Birmingham, Birmingham, United Kingdom

${ }^{22}$ Centro de Investigaciónes, Universidad Antonio Nariño, Bogota, Colombia

${ }^{23 a}$ Dipartimento di Fisica e Astronomia, Università di Bologna, Bologna, Italy

${ }^{23 \mathrm{~b}}$ INFN Sezione di Bologna, Italy

${ }^{24}$ Physikalisches Institut, Universität Bonn, Bonn, Germany

${ }^{25}$ Department of Physics, Boston University, Boston, Massachusetts, USA

${ }^{26}$ Department of Physics, Brandeis University, Waltham, Massachusetts, USA

${ }^{27}$ Transilvania University of Brasov, Brasov, Romania

${ }^{27 \mathrm{~b}}$ Horia Hulubei National Institute of Physics and Nuclear Engineering, Bucharest, Romania

${ }^{27 \mathrm{c}}$ Department of Physics, Alexandru Ioan Cuza University of Iasi, Iasi, Romania

${ }^{27 \mathrm{~d}}$ National Institute for Research and Development of Isotopic and Molecular Technologies,

Physics Department, Cluj-Napoca, Romania

${ }^{27 \mathrm{e}}$ University Politehnica Bucharest, Bucharest, Romania

${ }^{27 \mathrm{f}}$ West University in Timisoara, Timisoara, Romania

${ }^{28 a}$ Faculty of Mathematics, Physics and Informatics, Comenius University, Bratislava, Slovak Republic

${ }^{28 \mathrm{~b}}$ Department of Subnuclear Physics, Institute of Experimental Physics of the Slovak Academy of Sciences,

Kosice, Slovak Republic

${ }^{29}$ Physics Department, Brookhaven National Laboratory, Upton, New York, USA

${ }^{30}$ Departamento de Física, Universidad de Buenos Aires, Buenos Aires, Argentina

${ }^{31}$ Cavendish Laboratory, University of Cambridge, Cambridge, United Kingdom

${ }^{32 \mathrm{a}}$ Department of Physics, University of Cape Town, Cape Town, South Africa

${ }^{32 \mathrm{~b}}$ Department of Mechanical Engineering Science, University of Johannesburg, Johannesburg, South Africa

${ }^{32 c}$ School of Physics, University of the Witwatersrand, Johannesburg, South Africa

${ }^{33}$ Department of Physics, Carleton University, Ottawa, Ontario, Canada

${ }^{34 a}$ Faculté des Sciences Ain Chock, Réseau Universitaire de Physique des Hautes Energies-Université Hassan II, Casablanca, Morocco

${ }^{34 \mathrm{~b}}$ Centre National de l'Energie des Sciences Techniques Nucleaires (CNESTEN), Rabat, Morocco

${ }^{34 \mathrm{c}}$ Faculté des Sciences Semlalia, Université Cadi Ayyad, LPHEA-Marrakech, Morocco

${ }^{34 \mathrm{~d}}$ Faculté des Sciences, Université Mohamed Premier and LPTPM, Oujda, Morocco

${ }^{34 \mathrm{e}}$ Faculté des sciences, Université Mohammed V, Rabat, Morocco

${ }^{35}$ CERN, Geneva, Switzerland

${ }^{36}$ Enrico Fermi Institute, University of Chicago, Chicago, Illinois, USA

${ }^{37}$ LPC, Université Clermont Auvergne, CNRS/IN2P3, Clermont-Ferrand, France

${ }^{38}$ Nevis Laboratory, Columbia University, Irvington, New York, USA

${ }^{39}$ Niels Bohr Institute, University of Copenhagen, Copenhagen, Denmark

${ }^{40 \mathrm{a}}$ Dipartimento di Fisica, Università della Calabria, Rende, Italy 
${ }^{40 \mathrm{~b}}$ INFN Gruppo Collegato di Cosenza, Laboratori Nazionali di Frascati, Italy

${ }^{41}$ Physics Department, Southern Methodist University, Dallas, Texas, USA

${ }^{42}$ Physics Department, University of Texas at Dallas, Richardson, Texas, USA

${ }^{43 a}$ Department of Physics, Stockholm University, Sweden

${ }^{43 b}$ Oskar Klein Centre, Stockholm, Sweden

${ }^{44}$ Deutsches Elektronen-Synchrotron DESY, Hamburg and Zeuthen, Germany

${ }^{45}$ Lehrstuhl für Experimentelle Physik IV, Technische Universität Dortmund, Dortmund, Germany

${ }^{46}$ Institut für Kern- und Teilchenphysik, Technische Universität Dresden, Dresden, Germany

${ }^{47}$ Department of Physics, Duke University, Durham, North Carolina, USA

${ }^{48}$ SUPA - School of Physics and Astronomy, University of Edinburgh, Edinburgh, United Kingdom

${ }^{49}$ INFN e Laboratori Nazionali di Frascati, Frascati, Italy

${ }^{50}$ Physikalisches Institut, Albert-Ludwigs-Universität Freiburg, Freiburg, Germany

${ }^{51}$ II. Physikalisches Institut, Georg-August-Universität Göttingen, Göttingen, Germany

${ }^{52}$ Département de Physique Nucléaire et Corpusculaire, Université de Genève, Genève, Switzerland

${ }^{53}$ Dipartimento di Fisica, Università di Genova, Genova, Italy

${ }^{53 \mathrm{~b}}$ INFN Sezione di Genova, Italy

${ }^{54}$ II. Physikalisches Institut, Justus-Liebig-Universität Giessen, Giessen, Germany

${ }^{55}$ SUPA - School of Physics and Astronomy, University of Glasgow, Glasgow, United Kingdom

${ }^{56}$ LPSC, Université Grenoble Alpes, CNRS/IN2P3, Grenoble INP, Grenoble, France

${ }^{57}$ Laboratory for Particle Physics and Cosmology, Harvard University, Cambridge, Massachusetts, USA

${ }^{58 a}$ Department of Modern Physics and State Key Laboratory of Particle Detection and Electronics,

University of Science and Technology of China, Hefei, China

${ }^{58 \mathrm{~b}}$ Institute of Frontier and Interdisciplinary Science and Key Laboratory of Particle Physics and Particle Irradiation (MOE), Shandong University, Qingdao, China

${ }^{58 \mathrm{c}}$ School of Physics and Astronomy, Shanghai Jiao Tong University, KLPPAC-MoE, SKLPPC, Shanghai, China

${ }^{58 \mathrm{~d}}$ Tsung-Dao Lee Institute, Shanghai, China

${ }^{59 a}$ Kirchhoff-Institut für Physik, Ruprecht-Karls-Universität Heidelberg, Heidelberg, Germany

${ }^{59 \mathrm{~b}}$ Physikalisches Institut, Ruprecht-Karls-Universität Heidelberg, Heidelberg, Germany

${ }^{60}$ Faculty of Applied Information Science, Hiroshima Institute of Technology, Hiroshima, Japan

${ }^{61 a}$ Department of Physics, Chinese University of Hong Kong, Shatin, N.T., Hong Kong, China

${ }^{61 \mathrm{~b}}$ Department of Physics, University of Hong Kong, Hong Kong, China

${ }^{61 \mathrm{c} D e p a r t m e n t}$ of Physics and Institute for Advanced Study, Hong Kong University of Science and Technology,

Clear Water Bay, Kowloon, Hong Kong, China

${ }^{62}$ Department of Physics, National Tsing Hua University, Hsinchu, Taiwan

${ }^{63}$ Department of Physics, Indiana University, Bloomington, Indiana, USA

${ }^{64 a}$ INFN Gruppo Collegato di Udine, Sezione di Trieste, Udine, Italy

${ }^{64 \mathrm{~b}}$ ICTP, Trieste, Italy

${ }^{64 \mathrm{c}}$ Dipartimento di Chimica, Fisica e Ambiente, Università di Udine, Udine, Italy

${ }^{65}$ INFN Sezione di Lecce, Italy

${ }^{65 \mathrm{~b}}$ Dipartimento di Matematica e Fisica, Università del Salento, Lecce, Italy

${ }^{66 a}$ INFN Sezione di Milano, Italy

${ }^{66 \mathrm{~b}}$ Dipartimento di Fisica, Università di Milano, Milano, Italy

${ }^{67 a}$ INFN Sezione di Napoli, Italy

${ }^{67 \mathrm{~b}}$ Dipartimento di Fisica, Università di Napoli, Napoli, Italy

${ }^{68 \mathrm{a}}$ INFN Sezione di Pavia, Italy

${ }^{68 \mathrm{~b}}$ Dipartimento di Fisica, Università di Pavia, Pavia, Italy

${ }^{69 a}$ INFN Sezione di Pisa, Italy

${ }^{69 \mathrm{~b}}$ Dipartimento di Fisica E. Fermi, Università di Pisa, Pisa, Italy

${ }^{70 a}$ INFN Sezione di Roma, Italy

${ }^{70 \mathrm{~b}}$ Dipartimento di Fisica, Sapienza Università di Roma, Roma, Italy

${ }^{71 a}$ INFN Sezione di Roma Tor Vergata, Italy

${ }^{71 b}$ Dipartimento di Fisica, Università di Roma Tor Vergata, Roma, Italy

${ }^{72 \mathrm{a}}$ INFN Sezione di Roma Tre, Italy

${ }^{72 b}$ Dipartimento di Matematica e Fisica, Università Roma Tre, Roma, Italy

${ }^{73 a}$ INFN-TIFPA, Italy

${ }^{73 b}$ Università degli Studi di Trento, Trento, Italy

${ }^{74}$ Institut für Astro- und Teilchenphysik, Leopold-Franzens-Universität, Innsbruck, Austria

${ }^{75}$ University of Iowa, Iowa, City Iowa, USA

${ }^{76}$ Department of Physics and Astronomy, Iowa State University, Ames, Iowa, USA

${ }^{77}$ Joint Institute for Nuclear Research, Dubna, Russia 
${ }^{78 a}$ Departamento de Engenharia Elétrica, Universidade Federal de Juiz de Fora (UFJF), Juiz de Fora, Brazil

${ }^{78 b}$ Universidade Federal do Rio De Janeiro COPPE/EE/IF, Rio de Janeiro, Brazil

${ }^{78 c}$ Universidade Federal de São João del Rei (UFSJ), São João del Rei, Brazil

${ }^{78 \mathrm{~d}}$ Instituto de Física, Universidade de São Paulo, São Paulo, Brazil

${ }^{79}$ KEK, High Energy Accelerator Research Organization, Tsukuba, Japan

${ }^{80}$ Graduate School of Science, Kobe University, Kobe, Japan

${ }^{81 \mathrm{a}}$ AGH University of Science and Technology, Faculty of Physics and Applied Computer Science, Krakow, Poland

${ }^{81 \mathrm{~b}}$ Marian Smoluchowski Institute of Physics, Jagiellonian University, Krakow, Poland

${ }^{82}$ Institute of Nuclear Physics Polish Academy of Sciences, Krakow, Poland

${ }^{83}$ Faculty of Science, Kyoto University, Kyoto, Japan

${ }^{84}$ Kyoto University of Education, Kyoto, Japan

${ }^{85}$ Research Center for Advanced Particle Physics and Department of Physics, Kyushu University, Fukuoka, Japan

${ }^{86}$ Instituto de Física La Plata, Universidad Nacional de La Plata and CONICET, La Plata, Argentina

${ }^{87}$ Physics Department, Lancaster University, Lancaster, United Kingdom

${ }^{88}$ Oliver Lodge Laboratory, University of Liverpool, Liverpool, United Kingdom

${ }^{89}$ Department of Experimental Particle Physics, Jožef Stefan Institute and Department of Physics, University of Ljubljana, Ljubljana, Slovenia

${ }^{90}$ School of Physics and Astronomy, Queen Mary University of London, London, United Kingdom

${ }^{91}$ Department of Physics, Royal Holloway University of London, Egham, United Kingdom

${ }^{92}$ Department of Physics and Astronomy, University College London, London, United Kingdom

${ }^{93}$ Louisiana Tech University, Ruston, Louisiana, USA

${ }^{94}$ Fysiska institutionen, Lunds universitet, Lund, Sweden

${ }^{95}$ Centre de Calcul de l'Institut National de Physique Nucléaire et de Physique des Particules (IN2P3), Villeurbanne, France

${ }^{96}$ Departamento de Física Teorica C-15 and CIAFF, Universidad Autónoma de Madrid, Madrid, Spain

${ }^{97}$ Institut für Physik, Universität Mainz, Mainz, Germany

${ }^{98}$ School of Physics and Astronomy, University of Manchester, Manchester, United Kingdom

${ }^{99}$ CPPM, Aix-Marseille Université, CNRS/IN2P3, Marseille, France

${ }^{100}$ Department of Physics, University of Massachusetts, Amherst, Massachusetts, USA

${ }^{101}$ Department of Physics, McGill University, Montreal, Quebec, Canada

${ }^{102}$ School of Physics, University of Melbourne, Victoria, Australia

${ }^{103}$ Department of Physics, University of Michigan, Ann Arbor, Michigan, USA

${ }^{104}$ Department of Physics and Astronomy, Michigan State University, East Lansing, Michigan, USA

${ }^{105}$ B.I. Stepanov Institute of Physics, National Academy of Sciences of Belarus, Minsk, Belarus

${ }^{106}$ Research Institute for Nuclear Problems of Byelorussian State University, Minsk, Belarus

${ }^{107}$ Group of Particle Physics, University of Montreal, Montreal, Quebec, Canada

${ }^{108}$ P.N. Lebedev Physical Institute of the Russian Academy of Sciences, Moscow, Russia

${ }^{109}$ Institute for Theoretical and Experimental Physics (ITEP), Moscow, Russia

${ }^{110}$ National Research Nuclear University MEPhI, Moscow, Russia

${ }^{111}$ D.V. Skobeltsyn Institute of Nuclear Physics, M.V. Lomonosov Moscow State University, Moscow, Russia

${ }^{112}$ Fakultät für Physik, Ludwig-Maximilians-Universität München, München, Germany

${ }^{113}$ Max-Planck-Institut für Physik (Werner-Heisenberg-Institut), München, Germany

${ }^{114}$ Nagasaki Institute of Applied Science, Nagasaki, Japan

${ }^{115}$ Graduate School of Science and Kobayashi-Maskawa Institute, Nagoya University, Nagoya, Japan

${ }^{116}$ Department of Physics and Astronomy, University of New Mexico, Albuquerque, New Mexico, USA

${ }^{117}$ Institute for Mathematics, Astrophysics and Particle Physics, Radboud University Nijmegen/Nikhef, Nijmegen, Netherlands

${ }^{118}$ Nikhef National Institute for Subatomic Physics and University of Amsterdam, Amsterdam, Netherlands

${ }^{119}$ Department of Physics, Northern Illinois University, DeKalb, Illinois, USA

${ }^{120 a}$ Budker Institute of Nuclear Physics, SB RAS, Novosibirsk, Russia

${ }^{120 \mathrm{~b}}$ Novosibirsk State University Novosibirsk, Russia

${ }^{121}$ Department of Physics, New York University, New York, New York, USA

${ }^{122}$ Ohio State University, Columbus, Ohio, USA

${ }^{123}$ Faculty of Science, Okayama University, Okayama, Japan

${ }^{124}$ Homer L. Dodge Department of Physics and Astronomy, University of Oklahoma, Norman, Oklahoma, USA

${ }^{125}$ Department of Physics, Oklahoma State University, Stillwater, Oklahoma, USA

${ }^{126}$ Palacký University, RCPTM, Joint Laboratory of Optics, Olomouc, Czech Republic

${ }^{127}$ Center for High Energy Physics, University of Oregon, Eugene, Oregon, USA

${ }^{128}$ LAL, Université Paris-Sud, CNRS/IN2P3, Université Paris-Saclay, Orsay, France

${ }^{129}$ Graduate School of Science, Osaka University, Osaka, Japan 


\author{
${ }^{130}$ Department of Physics, University of Oslo, Oslo, Norway \\ ${ }^{131}$ Department of Physics, Oxford University, Oxford, United Kingdom \\ ${ }^{132}$ LPNHE, Sorbonne Université, Paris Diderot Sorbonne Paris Cité, CNRS/IN2P3, Paris, France \\ ${ }^{133}$ Department of Physics, University of Pennsylvania, Philadelphia, Pennsylvania, USA \\ ${ }^{134}$ Konstantinov Nuclear Physics Institute of National Research Centre "Kurchatov Institute”, \\ PNPI, St. Petersburg, Russia \\ ${ }^{135}$ Department of Physics and Astronomy, University of Pittsburgh, Pittsburgh, Pennsylvania, USA \\ ${ }^{136 a}$ Laboratório de Instrumentação e Física Experimental de Partículas - LIP, Portugal \\ ${ }^{136 \mathrm{~b}}$ Departamento de Física, Faculdade de Ciências, Universidade de Lisboa, Lisboa, Portugal \\ ${ }^{136 \mathrm{c}}$ Departamento de Física, Universidade de Coimbra, Coimbra, Portugal \\ ${ }^{136 \mathrm{~d}}$ Centro de Física Nuclear da Universidade de Lisboa, Lisboa, Portugal \\ ${ }^{136 e}$ Departamento de Física, Universidade do Minho, Braga, Portugal \\ ${ }^{136 f}$ Departamento de Física Teorica y del Cosmos, Universidad de Granada, Granada (Spain), Spain \\ ${ }^{136 \mathrm{~g}}$ Dep Física and CEFITEC of Faculdade de Ciências e Tecnologia, Universidade Nova de Lisboa, Caparica, Portugal \\ ${ }^{137}$ Institute of Physics, Academy of Sciences of the Czech Republic, Prague, Czech Republic \\ ${ }^{138}$ Czech Technical University in Prague, Prague, Czech Republic \\ ${ }^{139}$ Charles University, Faculty of Mathematics and Physics, Prague, Czech Republic \\ ${ }^{140}$ State Research Center Institute for High Energy Physics, NRC KI, Protvino, Russia \\ ${ }^{141}$ Particle Physics Department, Rutherford Appleton Laboratory, Didcot, United Kingdom \\ ${ }^{142}$ IRFU, CEA, Université Paris-Saclay, Gif-sur-Yvette, France \\ ${ }^{143}$ Santa Cruz Institute for Particle Physics, University of California Santa Cruz, Santa, Cruz, California, USA \\ ${ }^{144 a}$ Departamento de Física, Pontificia Universidad Católica de Chile, Santiago, Chile \\ ${ }^{144 b}$ Departamento de Física, Universidad Técnica Federico Santa María, Valparaíso, Chile \\ ${ }^{145}$ Department of Physics, University of Washington, Seattle, Washington, USA \\ ${ }^{146}$ Department of Physics and Astronomy, University of Sheffield, Sheffield, United Kingdom \\ ${ }^{147}$ Department of Physics, Shinshu University, Nagano, Japan \\ ${ }^{148}$ Department Physik, Universität Siegen, Siegen, Germany \\ ${ }^{149}$ Department of Physics, Simon Fraser University, Burnaby British Columbia, Canada \\ ${ }^{150}$ SLAC National Accelerator Laboratory, Stanford, California, USA \\ ${ }^{151}$ Physics Department, Royal Institute of Technology, Stockholm, Sweden \\ ${ }^{152}$ Departments of Physics and Astronomy, Stony Brook University, Stony Brook, New York, USA \\ ${ }^{153}$ Department of Physics and Astronomy, University of Sussex, Brighton, United Kingdom \\ ${ }^{154}$ School of Physics, University of Sydney, Sydney, Australia \\ ${ }^{155}$ Institute of Physics, Academia Sinica, Taipei, Taiwan \\ ${ }^{156}$ Academia Sinica Grid Computing, Institute of Physics, Academia Sinica, Taipei, Taiwan \\ ${ }^{157 a}$ E. Andronikashvili Institute of Physics, Iv. Javakhishvili Tbilisi State University, Tbilisi, Georgia \\ ${ }^{157 b}$ High Energy Physics Institute, Tbilisi State University, Tbilisi, Georgia \\ ${ }^{158}$ Department of Physics, Technion, Israel Institute of Technology, Haifa, Israel \\ ${ }^{159}$ Raymond and Beverly Sackler School of Physics and Astronomy, Tel Aviv University, Tel Aviv, Israel \\ ${ }^{160}$ Department of Physics, Aristotle University of Thessaloniki, Thessaloniki, Greece \\ ${ }^{161}$ International Center for Elementary Particle Physics and Department of Physics, University of Tokyo, Tokyo, Japan \\ ${ }^{162}$ Graduate School of Science and Technology, Tokyo Metropolitan University, Tokyo, Japan \\ ${ }^{163}$ Department of Physics, Tokyo Institute of Technology, Tokyo, Japan \\ ${ }^{164}$ Tomsk State University, Tomsk, Russia \\ ${ }^{165}$ Department of Physics, University of Toronto, Toronto, Ontario, Canada \\ ${ }^{166 a}$ TRIUMF, Vancouver, British Columbia, Canada \\ ${ }^{166 \mathrm{~b}}$ Department of Physics and Astronomy, York University, Toronto, Ontario, Canada \\ ${ }^{167}$ Division of Physics and Tomonaga Center for the History of the Universe, Faculty of Pure and Applied Sciences, \\ University of Tsukuba, Tsukuba, Japan \\ ${ }^{168}$ Department of Physics and Astronomy, Tufts University, Medford, Massachusetts, USA \\ ${ }^{169}$ Department of Physics and Astronomy, University of California Irvine, Irvine, California, USA \\ ${ }^{170}$ Department of Physics and Astronomy, University of Uppsala, Uppsala, Sweden \\ ${ }^{171}$ Department of Physics, University of Illinois, Urbana, Illinois, USA \\ ${ }^{172}$ Instituto de Física Corpuscular (IFIC), Centro Mixto Universidad de Valencia - CSIC, Valencia, Spain \\ ${ }^{173}$ Department of Physics, University of British Columbia, Vancouver, British Columbia, Canada \\ ${ }^{174}$ Department of Physics and Astronomy, University of Victoria, Victoria, British Columbia, Canada \\ ${ }^{175}$ Fakultät für Physik und Astronomie, Julius-Maximilians-Universität Würzburg, Würzburg, Germany \\ ${ }^{176}$ Department of Physics, University of Warwick, Coventry, United Kingdom \\ ${ }^{177}$ Waseda University, Tokyo, Japan \\ ${ }^{178}$ Department of Particle Physics, Weizmann Institute of Science, Rehovot, Israel
}




\section{${ }^{179}$ Department of Physics, University of Wisconsin, Madison, Wisconsin, USA \\ ${ }^{180}$ Fakultät für Mathematik und Naturwissenschaften, Fachgruppe Physik, Bergische Universität Wuppertal, Wuppertal, Germany \\ ${ }^{181}$ Department of Physics, Yale University, New Haven, Connecticut, USA \\ ${ }^{182}$ Yerevan Physics Institute, Yerevan, Armenia}

${ }^{\mathrm{a}}$ Deceased.

${ }^{\mathrm{b}}$ Also at Department of Physics, King's College London, London, United Kingdom.

${ }^{c}$ Also at Institute of Physics, Azerbaijan Academy of Sciences, Baku, Azerbaijan.

${ }^{\mathrm{d}}$ Also at TRIUMF, Vancouver, British Columbia, Canada.

${ }^{\mathrm{e}}$ Also at Department of Physics and Astronomy, University of Louisville, Louisville, Kentucky, USA.

${ }^{\mathrm{f}}$ Also at Department of Physics, California State University, Fresno, California, USA.

${ }^{g}$ Also at Department of Physics, University of Fribourg, Fribourg, Switzerland.

${ }^{h}$ Also at II. Physikalisches Institut, Georg-August-Universität Göttingen, Göttingen, Germany.

${ }^{\mathrm{i}}$ Also at Departament de Fisica de la Universitat Autonoma de Barcelona, Barcelona, Spain.

${ }^{\mathrm{j}}$ Also at Tomsk State University, Tomsk, and Moscow Institute of Physics and Technology State University, Dolgoprudny, Russia.

${ }^{\mathrm{k}}$ Also at The Collaborative Innovation Center of Quantum Matter (CICQM), Beijing, China.

${ }^{1}$ Also at Universita di Napoli Parthenope, Napoli, Italy.

${ }^{\mathrm{m}}$ Also at Institute of Particle Physics (IPP), Canada.

${ }^{\mathrm{n}}$ Also at Dipartimento di Fisica E. Fermi, Università di Pisa, Pisa, Italy.

${ }^{\circ}$ Also at Horia Hulubei National Institute of Physics and Nuclear Engineering, Bucharest, Romania.

${ }^{\mathrm{p}}$ Also at CPPM, Aix-Marseille Université, CNRS/IN2P3, Marseille, France.

${ }^{\mathrm{q}}$ Also at Department of Physics, St. Petersburg State Polytechnical University, St. Petersburg, Russia.

${ }^{\mathrm{r}}$ Also at Borough of Manhattan Community College, City University of New York, New York, USA.

${ }^{\mathrm{s}}$ Also at Department of Financial and Management Engineering, University of the Aegean, Chios, Greece.

${ }^{\mathrm{t}}$ Also at Centre for High Performance Computing, CSIR Campus, Rosebank, Cape Town, South Africa.

"Also at Louisiana Tech University, Ruston, Louisiana, USA.

${ }^{v}$ Also at Institucio Catalana de Recerca i Estudis Avancats, ICREA, Barcelona, Spain.

${ }^{\mathrm{w}}$ Also at Department of Physics, University of Michigan, Ann Arbor, Michigan, USA.

${ }^{\mathrm{x}}$ Also at LAL, Université Paris-Sud, CNRS/IN2P3, Université Paris-Saclay, Orsay, France.

${ }^{y}$ Also at Graduate School of Science, Osaka University, Osaka, Japan.

${ }^{\mathrm{z}}$ Also at Physikalisches Institut, Albert-Ludwigs-Universität Freiburg, Freiburg, Germany.

${ }^{\text {aa }}$ Also at Institute for Mathematics, Astrophysics and Particle Physics, Radboud University Nijmegen/Nikhef, Nijmegen, Netherlands.

${ }^{\mathrm{bb}}$ Also at Near East University, Nicosia, North Cyprus, Mersin, Turkey.

${ }^{c c}$ Also at Institute of Theoretical Physics, Ilia State University, Tbilisi, Georgia.

${ }^{\mathrm{dd}}$ Also at CERN, Geneva, Switzerland.

${ }^{\mathrm{ee}}$ Also at Manhattan College, New York, New York, USA.

${ }^{\mathrm{ff}}$ Also at Hellenic Open University, Patras, Greece.

${ }^{\mathrm{gg}}$ Also at The City College of New York, New York, New York, USA.

${ }^{\text {hh }}$ Also at Departamento de Física Teorica y del Cosmos, Universidad de Granada, Granada (Spain), Spain.

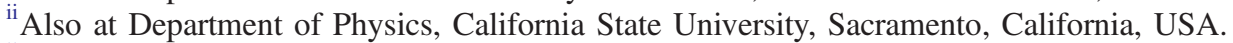

${ }^{\mathrm{jj}}$ Also at Moscow Institute of Physics and Technology State University, Dolgoprudny, Russia.

${ }^{\mathrm{kk}}$ Also at Ochadai Academic Production, Ochanomizu University, Tokyo, Japan.

${ }^{11}$ Also at Département de Physique Nucléaire et Corpusculaire, Université de Genève, Genève, Switzerland.

${ }^{\mathrm{mm}}$ Also at Department of Physics and Astronomy, University of Sheffield, Sheffield, United Kingdom.

${ }^{n n}$ Also at School of Physics, Sun Yat-sen University, Guangzhou, China.

${ }^{\circ o}$ Also at Department of Applied Physics and Astronomy, University of Sharjah, Sharjah, United Arab Emirates.

${ }^{\mathrm{pp}}$ Also at Institut für Experimentalphysik, Universität Hamburg, Hamburg, Germany.

${ }^{\mathrm{qq}}$ Also at National Research Nuclear University MEPhI, Moscow, Russia.

${ }^{\mathrm{rr}}$ Also at Institute for Particle and Nuclear Physics, Wigner Research Centre for Physics, Budapest, Hungary.

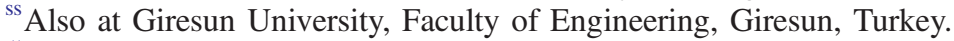

${ }^{t t}$ Also at Department of Physics, Nanjing University, Nanjing, China.

${ }^{u u}$ Also at Institute of Physics, Academia Sinica, Taipei, Taiwan.

${ }^{\mathrm{vv}}$ Also at Department of Physics, University of Malaya, Kuala Lumpur, Malaysia. 